\title{
An Extensive Survey on the Internet of Drones
}

\author{
Pietro Boccadoro, IEEE Member ${ }^{\mathrm{a}}$, Domenico Striccolia ${ }^{\mathrm{a}}$, Luigi Alfredo Grieco, \\ IEEE Member ${ }^{\mathrm{a}}$ \\ ${ }^{a}$ Dep. of Electrical and Information Engineering (DEI), Politecnico di Bari, via Orabona \\ 4, Bari, 70126, Bari, Italy, email: name.surname@poliba.it
}

\begin{abstract}
The Internet of Drones (IoD) recently gained momentum due to its high adaptability to a wide variety of complex scenarios. Indeed, Unmanned Aerial Vehicles (UAVs) can successfully be employed in different applications, such as agriculture, search and rescue missions, surveillance systems, mission-critical services, etc., thanks to some technological and practical advantages: high mobility, capability to extend wireless coverage areas, or ability to reach places inaccessible to humans. Moreover, the employment of drones promisingly improves the performance parameters of different network architectures, i.e., reliability, connectivity, throughput, and delay, among others. Nevertheless, the adoption of networks of drones gives rise to several issues related to the unreliability of the wireless medium, the duration of batteries, and the high mobility degree, which may cause frequent topology changes. Also security and privacy issues need to be properly investigated. This explains the very large number of works produced in the recent literature on IoD-related topics. With respect to other surveys on IoD-related topics, the goal of the present work is to categorize the multifaceted aspects of IoD, proposing a classification approach of the IoD environment that develops along two main directions. At a macroscopic level, it follows the structure of the Internet protocol stack, starting from the physical layer and extending to the upper layers, without neglecting cross-layer and optimization approaches. At a finer level, all the most relevant works belonging to each layer of the stack are further classified, according to the different issues peculiar of the layer, and highlighting the most relevant differences with the other surveys present in literature. To provide a deeper insight in the theme, the present work embraces many facets of the IoD, including privacy and security considerations as well as the potential economic impact of the IoD. Finally, a discussion on the main research challenges and possible future directions is carried out, focusing on the open issues and the most promising technologies that deserve to be further developed in the IoD field.
\end{abstract}


Keywords: Internet of Drones, Unmanned Aerial Vehicle, Air-to-Air, Air-to-Ground, 5G, mmWave, Visible Light Communication

\section{Introduction}

The IoD is defined as a network architecture specifically aimed at supporting communications between autonomous vehicles able to fly and a number of network entities deployed on the ground [1, 2]. In the IoD, drones are conceived as smart objects in charge of flying all over a certain area, to carry out a number of different tasks, spanning from patrolling to sensing environmental data, in order to gather data of interest, even in real-time, for any further use. In the IoD architecture, UAVs are conceived as networked objects able to communicate among themselves, exchanging data, for example connected with flight coordination capabilities. At the same time, drones are communicating with a reference ground infrastructure that is in charge of storing and elaborating data for enabling services or providing updated information to remote users connected with dedicated application servers. The reference ground infrastructure is also in charge of controlling the airspace, granting the optimal position of drones and monitoring their activities. Since the ground network infrastructure is conceived as a set of logical nodes with high computational capabilities, tasks coordination and mission plan updates can be delegated, without affecting the limited resources onboard of drones.

IoD has attracted much interest in the recent literature, due to the flexibility and the adaptability of networks of drones in the most widely differing scenarios, and their ability of enhance the performance of other network architectures. UAVs are becoming more and more widespread in many fields, due to some technological, tactical and/or practical advantages: (i) high mobility, (ii) easy deployment and re-employability, (iii) real-time monitoring and coordination (strongly dependent on both system architectures and communication technologies), (iv) load transport (depending on the specific cargo application requirements). Drones can also be employed in places hardly accessible to humans, enhancing the network connectivity, coverage and capacity, especially in combination with other wireless/wired network architectures [1, 2, 3, 4]. Given this wide range of functionalities, a number of different applications for the IoD can be enabled. Among them: smart agriculture, search and rescue, surveillance systems, mission-critical services, stock management, sport and training, telecommunications, art and creativity, etc. [3].

Against this, several issues need to be properly investigated in $\mathrm{IoD}$ scenarios. First, the wireless medium by its own nature is unreliable: it is prone to errors, 
attenuations, and multipath propagation, thus bringing to data losses and link interruptions. Even though this is a well-known aspect of wireless communications, this becomes more important when the networked objects are flying by design and moving suddenly from one place to another. This kind of problem has been tackled in literature when referring to Wireless Sensor Networks (WSNs), Mobile Ad-hoc NETworks (MANETs), and Flying Ad-hoc NETworks (FANETs). Unfortunately, in the IoD, drones are accomplishing several tasks, spanning from coverage enhancement, when they are employed as Flying Base Stations (FBSs), to pattern identification in crowd situations. In these cases, the intrinsic unreliability of the wireless medium suggests that dedicated design efforts must be devoted. In fact, the communication range is often limited, especially in environments with high signal attenuation [5]. The high mobility degree of drones makes this situation even worse, requiring an accurate control and coordination of drones fleets to avoid conflicts, especially in terms of routes and data exchange [6, 3].

Power consumption is another critical point in battery powered devices as drones are. This problem is not new to those that study Internet of Things (IoT), since sparing battery and consuming less energy when sensing, communicating, and elaborating data is a mandatory design criterion in that domain. This is motivated by the fact that in the IoT domain, network nodes are supposed to be deployed with low or null maintenance. As a consequence, policies of duty cycling and low transmission power are usually involved in this context. What makes the IoD a more challenging operating scenario is the fact that energy consumption is not only associated with sensing and communicating but also with movements and flight patterns and mission plans. Further, while IoT devices are often communicating low amounts of data, drones are frequently involved in high traffic volumes. This happens for example when drones are acting as FBSs or supporting video streaming applications. This suggests that onboard memory matters must be faced [7]. Efficient resource allocation should thus be employed to optimize energy consumption, in terms of both the energy spent for data communication and routes of drones [3]. In some application scenarios, where drones are used as relay points to collect and/or deliver data in multi-hop flying networks, cooperation is a mandatory requirement to increase network connectivity, coverage performance, latency and throughput. Moreover, frequent topology changes make much more difficult to implement effective routing protocols that keep a high level of data reliability and network connectivity [8, 4, 9]. Last but not least, the idea that drones can communicate logically implies some major concerns about security and privacy. Given the constraints in terms of energy consumption and computational capabilities the drones are logically affected by, lightweight cryp- 
tography and security solutions are highly recommendable [6]. Overall, the IoD is proposed to allow the development of networks composed by heterogeneous and interconnected drones with proper resource planning to be integrated within third parties businesses and services [10].

The relevance of IoD-related topics to the scientific community to solve the issues described above is testified by the very large number of works found in literature, where the IoD paradigm is analyzed in all its multifaceted aspects. The motivation for this survey is to analyze communications aspects, technologies, protocols and architectures in the IoD scenario, detailing as much as possible the analysis of the state-of-the-art research on the topic, at the same time updating and completing the analysis of the other surveys found in literature [4, 11, 8, 6, 3, 12, 5].

For all the reasons mentioned above, and for ease of comprehension, the classification proposed in this work follows, at a higher level, the classical protocol stack, starting from the analysis of all the issues related to the physical layer and going on with the data link layer, the network layer, and the transport and application layers. Nevertheless, there are also cross-layer and optimization approaches that cannot be framed in any of the protocol layers. They have thus been analyzed and discussed in a separate section.

The last part of this work is then focused on a discussion on the main research challenges and possible future directions in the IoD field.

Although researchers and research teams worldwide are concentrated on drones' related topics, all the themes that the scientific community is dealing with appear to be fragmented. As a result, all the works that will be described herein, as well as the survey papers, are only focused on specific aspects, leading to non-structured nor systematic studies. For instance, when discussing the application of drones in real world context, there is a lack of characterization of the operational contexts. Indeed, the social and economical impact is not discussed in detail. At the same time, these aspects are often treated on a regional basis. Moreover, in the context of drones communications, several solutions have been proposed in terms of modulation techniques. Nevertheless, the theme has never been surveyed before. To provide a further example, when optimization approaches are discussed, the proposed frameworks are not as thorough as they could be. For example, it is easy to find papers that optimize both trajectory and energy consumption. Sometimes it is possible to find papers that design the path followed by a drone during a mission without taking into consideration 3D spaces. Hence, while specific aspects are addressed within the other surveys and overviews on the IoD, the purpose of this work is to carry out an organic and thorough analysis, dealing with all the main 
aspects of the theme. At the same time, this work investigates the applicability of novel technologies, e.g., mmWave and Visible Light Communication (VLC), upcoming networks, i.e., Sixth-Generation (6G), and communication paradigms, i.e., Information-Centric Networking (ICN). To the best of authors knowledge, this is also the first study on the subject that also considers drone economics, thus discussing the economic impact of the adoption of drones in all the main application fields.

The remainder of this paper is organized as follows: Section 3 discusses the applicability of the IoD paradigm in real use-cases. Section 2 proposes a detailed study on the related surveys, describing their classification approach. At the same time, it clarifies the differences between the analytical models and approaches proposed so far and the way this work approaches the IoD, as a whole. Section 4 focuses on the physical layer, and provides a detailed characterization on connectivity issues as well as channel modeling approaches. Section 5 focuses on data link layer, discussing the related works on resource allocation and data scheduling. Section 6 is dedicated to network layer, paying attention mainly to coordination aspects, routing problems and relaying schemes. Section 7 is focused on the application layer, which includes Quality of Experience (QoE) indicators, computation offloading, task allocation and data collection/distribution. Section 8 analyzes all the papers proposing cross-layer approaches and optimization strategies in several IoD-related aspects, ranging from path planning and collision avoidance to network formation and control, energy efficiency, mobility, network architectures, etc. Section 9 is focused on security aspects and business models, which can be considered as complementary themes when discussing the development of drones as enabling technology toward the IoD. Section 10 summarizes the main findings and discusses the strengths and weaknesses of the analyzed technological landscape. All the considerations made in the surveyed literature pave the way of future research perspectives which are discussed in detail, with specific focus on the open issues and the most promising technologies. Finally, Section 11 concludes the work.

\section{Related Survey and Review Papers}

The main goal of this Section is to analyze all the available survey works related to the IoD and to IoD-related themes. The aim is to better highlight the key contributions of the present work with respect to what is already available in literature. The overall organization of this section is reported in Figure 1. 


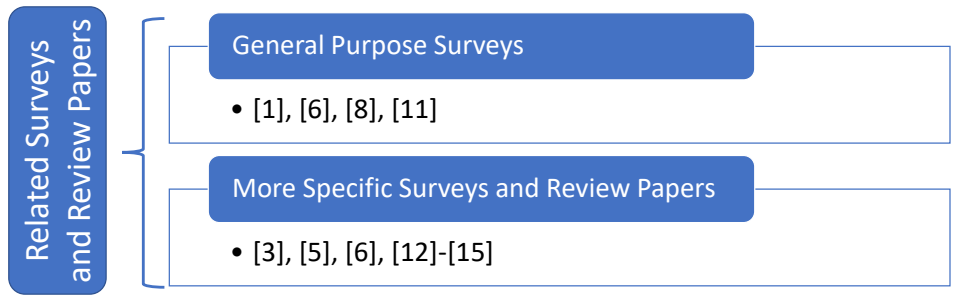

Figure 1: Related works Taxonomy.

\subsubsection{General Purpose Surveys}

There are some papers that investigate in detail several aspects related to networks of drones [1] [11] [8] [6].

The first, and probably most important, work on IoD can be found in [1]. It proposes an IoD architecture that aims to solve the problem of airspace allocation and management. Other network architectures are first analyzed, to derive valuable lessons on scalability and fault tolerance to be fruitfully exploited in networks of drones, and at the same time highlighting the main differences with the IoD scenario. The proposed architecture is then introduced, to provide generic services that can be used by different applications where drones are enabled to carry out tasks. Some challenges for effective IoD systems are also explored and discussed, to provide future research directions on the topic.

The integration of UAVs in cellular networks is analyzed in [6]. Several aspects are discussed in detail. First, UAVs types and main features (flying mechanisms, coverage range and altitude, speed, flight time, and power consumption) are addressed. Several standardization studies on channel modeling and characterization aspects are discussed in detail. The main part of this survey focuses on the different challenges and opportunities for UAV-based networks, ranging from the optimization of aerial Base Stations (BSs) location and flying path to the minimization of energy consumption. Different prototyping tests are also surveyed, together with the main aspects of UAV regulations and security issues.

Another interesting contribution is provided by [8]. It proposes a classification of UAVs based on their employability in the contexts of Vehicular Ad-hoc NETworks (VANETs) and MANETs, comparing such use cases with networks composed by UAVs only (ad-hoc networks). The contribution also highlights some of the main characteristics of UAV networks, taking into account single-UAV and multi-UAV systems. Networks are categorized based on their topology. A large part of this survey is dedicated to routing approaches. The work is completed with 
a detailed discussion on strategies for energy efficiency and handover.

In the survey [11] a detailed review of papers on communication technologies is presented for networks of drones in IoT scenarios. Literature is analyzed based on the classification of technologies into long-range and short-range. Long-range technologies are discussed in papers focusing on drones control, connectivity increase, relaying, and in emergency situations or when UAVs are outside the range of direct links. Short range technologies are exploited for different applications like flight control, real-time data acquisition, file transfer, device synchronization, low-power/low-cost communications, and UAV localization [11]. Literature is classified based on some application scenarios, i.e., monitoring activities, management of road traffic and disasters, path planning and routing, data collection mechanisms, almost exclusively referred to hardware (sensors, cameras, RFIDs, etc.) on board the drones. This survey also investigates the literature on network architectures and communication technologies, i.e., WiFi, satellite, WiMax, etc.

Even if each of the works [11, 8, 6] exhaustively analyzes aspects peculiar of UAV networks, nevertheless the discussion remains confined to specific topics, such as communication technologies in IoT [11], routing and energy efficiency [8], or legislation and security aspects in UAV-assisted cellular networks [6]. As a matter of fact, none of these surveys conducts a wide-range analysis of the main challenges present at different layers of the protocol stack.

\subsubsection{More Specific Surveys and Review Papers}

Even more contextualized to specific IoD-related topics is the analysis carried out in the survey and review papers $[3,5,13,14,12,15,6]$.

The survey [3] focuses on cooperation models for UAV-based networks. Single UAV and multi-UAV systems are analyzed in detail, with specific reference to relaying and routing strategies for cooperative network formation and coordination. The goal is to provide a detailed insight of the existing models, with their related features, to allow researchers to identify the existing solutions and analyze the performance of both existing and the models proposed. To this end, the available software solutions and simulators that enable cooperation and communications among flying entities forming ad-hoc networks are also compared and discussed, to test the implementation of an UAV-oriented network.

Physical layer aspects in the aerial/ground environment are analyzed in [5], with an emphasis on channel characterization and modeling. This work focuses mainly on the measurement approaches at the basis of the modeling procedures in UAV networks. A classification follows of the different kinds of models, empirical or analytical, through the analysis of the papers on the topic. Empirical 
studies based on measurement campaigns are described in detail, taking into account several factors like signal frequency, environmental conditions and UAVs main parameters (coverage, height, and mobility).

Robotic networks for surveillance purposes are discussed in [13]. A discussion is carried out on the main research directions and challenges of robotics (with a detailed analysis on autonomy issues), with an analysis of the acoustic signal treatment and processing, network protocols and security aspects in underwater communications. This work is interesting because it refers to a very hostile environment for wireless communications, as the underwater scenario is.

The overview work [14] deals exclusively with location optimization algorithms. It makes a classification of the algorithms that can be used for optimizing the position occupied by a certain number of UAVs, especially when they are supposed/asked to act as BS (the so-called Unmanned Aerial Vehicle Base Stations (UAV-BSs)). Different classes of algorithms are analyzed in this survey. They include exact approaches, able to find the global optimum of the optimization problem, as well as heuristic-based approaches, reinforcement learning approaches, or other searching techniques. This kind of classification is useful to solve the formulated problems through Mixed Integer Non-Linear Programming (MINLP) techniques.

The paper [12] overviews area coverage problems in UAV networks. The main impacting factors, i.e., coverage capabilities, UAVs mobility and lifetime, network connectivity, and presence of obstacles in the environment, are discussed with specific reference to the coverage types that are classified based on the UAVs motion (hover, fly, stall, etc.) and the network deployment. Other types of constraints in the drones utilization, i.e. energy consumption, coordination among UAVs and path planning problems, are summarily described together with path models adopted to study dynamic coverage problems.

The mmWave technology is analyzed in [15]. It overviews the issues arising from the utilization of mmWave communication in UAV networks. The main research challenges are discussed, especially with respect to channel modeling and estimation/acquisition strategies. The adoption of mmWave in cellular networks is also discussed with reference to aerial-to-ground communication issues that include both the UAV-to-BS and UAV-to-User Equipment (UE) scenarios. Scheduling and sharing of physical (time/frequency) resources among users are taken into account.

It is noteworthy that papers [3, 5, 13, 14, 12, 15] analyze a specific aspect of the IoD scenario: papers [3, 5] refer to the only network and physical layer respectively; the survey [13] deals with surveillance in the underwater environment. 
The works [14, 12] summarily review optimization strategies [14] and coverage problems [12]. The paper [15] is focused on the research perspectives in mmWave communication technologies.

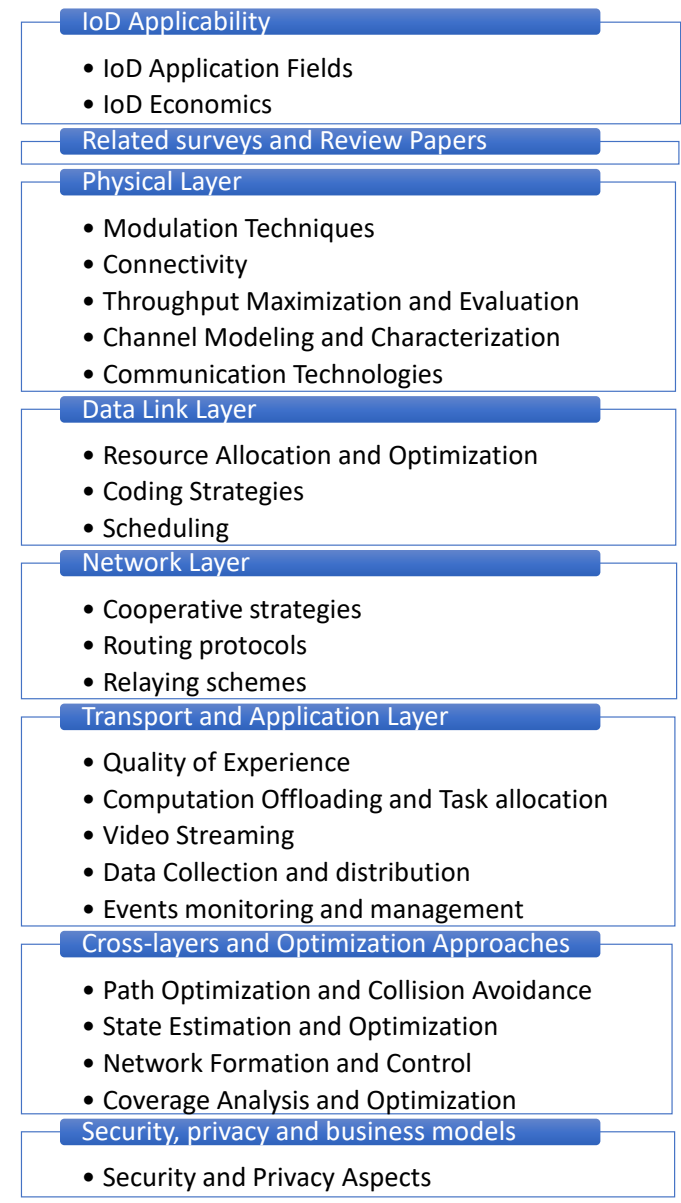

Figure 2: Overall organization of the work.

The discussion carried out so far reveals that there is the need to conduct a wider study on the theme. Since the reported works are mainly focused on a subset of the technological aspects of the $\mathrm{IoD}$, or specific phenomena, a more cross-cutting classification approach is needed. This is assumed by leveraging a thorough layer-by-layer characterization of mathematical problem formulations, technologies employment, and implementation proposals. As an outcome, the challenging issues and open research questions will be characterized in networks 


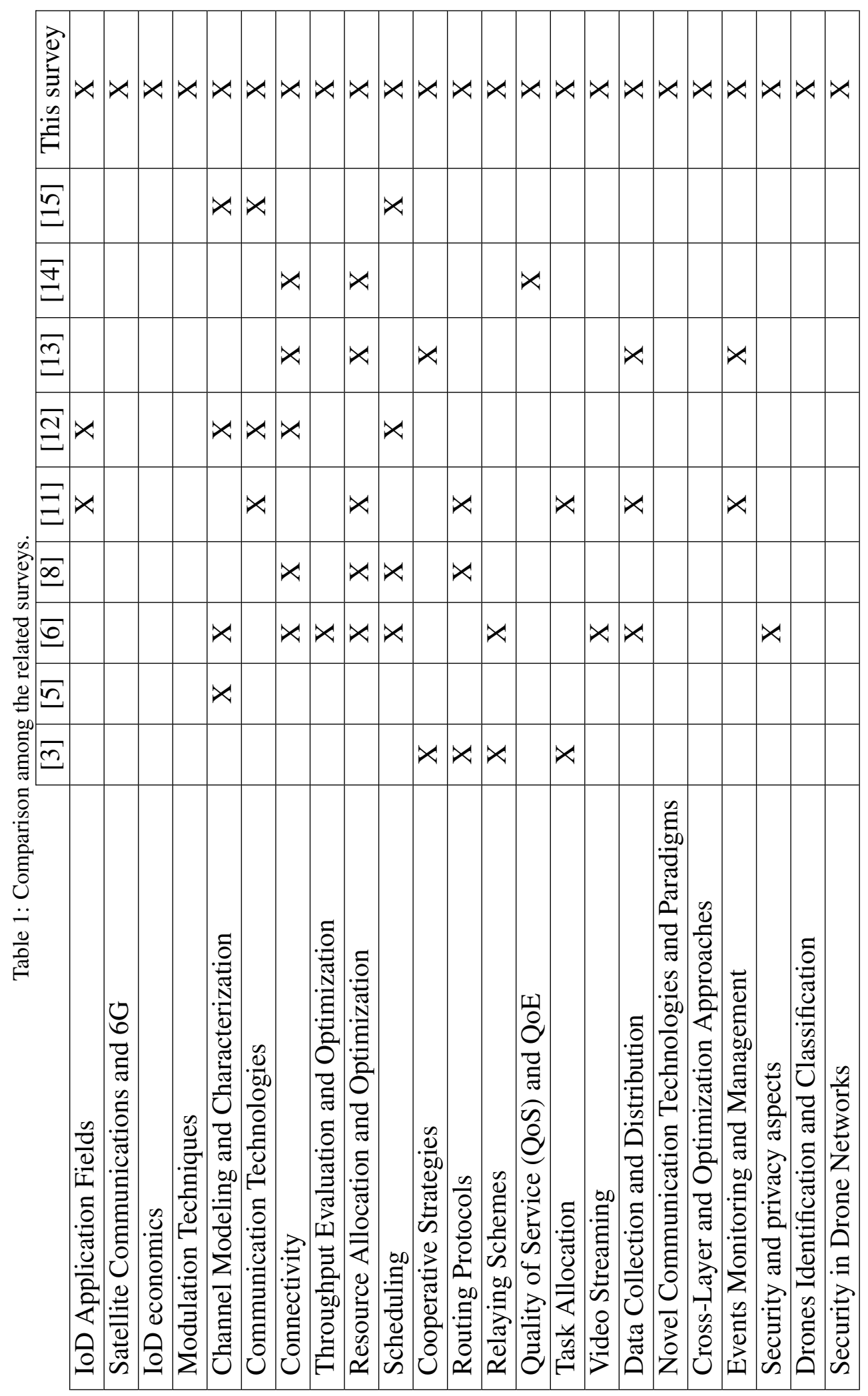


of drones, as remarked in Section 1. In Figure 2, the overall organization of the present contribution is shown. Furthermore, for each Section, a detailed representation of the taxonomy will be reported, showing its structure in detail. In Table 1. the surveyed state of the art is proposed, with a detailed overview of the theme that each work describes and tackles.

\section{IoD applicability}

This Section discusses the applicability of the IoD paradigm with a detailed analysis of all the application fields that have been proposed so far. To dig deeper in the subject, the employment of drones in the many applications that may benefit from their adoption are dealt with from an economical perspective. The overall organization of this section is reported in Figure 3 .

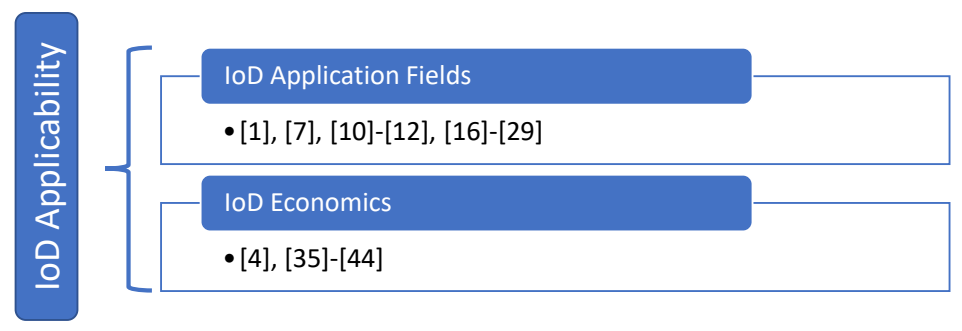

Figure 3: IoD Applicability Taxonomy.

\subsection{IoD Application Fields}

As previously anticipated, drones employability in different operational contexts has been widely discussed so far [1, 16, 17, 18, 19, 20, 10, 12, 21, 7, 22, 23, $11,24,25,26,27,28,29]$. In many cases, the studies propose a detailed analysis of their capabilities, the possibilities they enable, the persistent configurations and, above all, the involved technologies. In the telecommunications field, drones are of interest because they can be used both in emergency situations and in the ordinary conditions, i.e., management of data traffic. As an application example, let be an event with many connected users who want to send data through their $4 \mathrm{G}$ connections. In such a situation, the phone cells are typically overloaded. In this scenario drones can act as mobile BSs, delivering the service with smart and self-adaptive deployment plans to support the load in excess, thus granting connections continuity. These capabilities can also be extremely useful in those contexts where service interruptions and signal losses are due to network outages. 


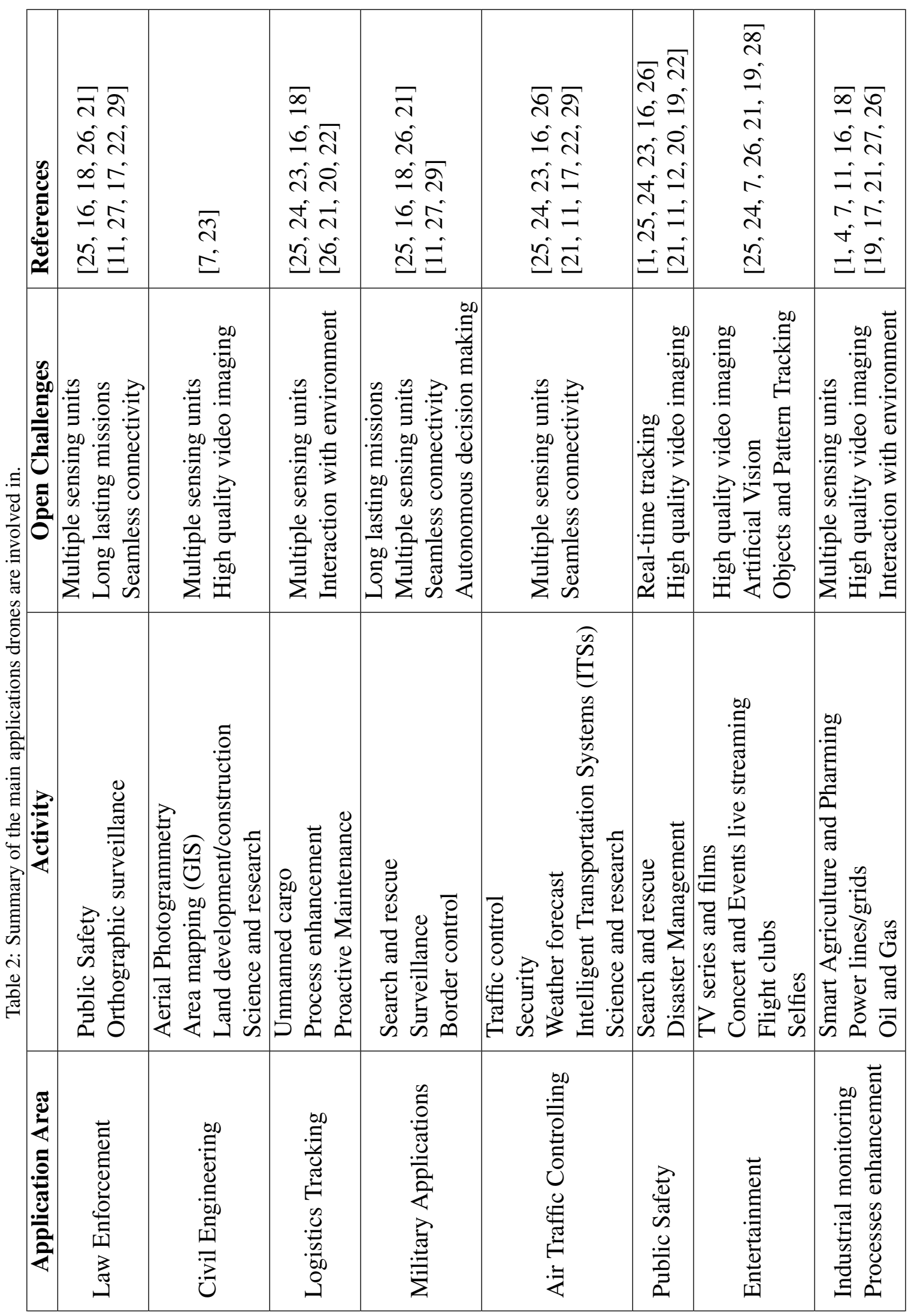


In case of environmental disasters, such as earthquakes and floods, the massive use of fleets of drones may be the only possibility of timely intervention to restore connectivity and in the meantime guarantees the acquisition of data, i.e., video images or environmental parameters. In non-critical scenarios, several examples of drones employment can be done, such as photogrammetry and aerial survey of both buildings and large wooded areas, as well as in smart agriculture. Here, the detailed analysis of high-resolution images can provide several kinds of information, like for example the quantity of pesticides or irrigation, the effects of abundant rain, the quality of the soil, the presence of diseases, etc. This information allows targeted interventions to save entire crops and improve the quality of production.

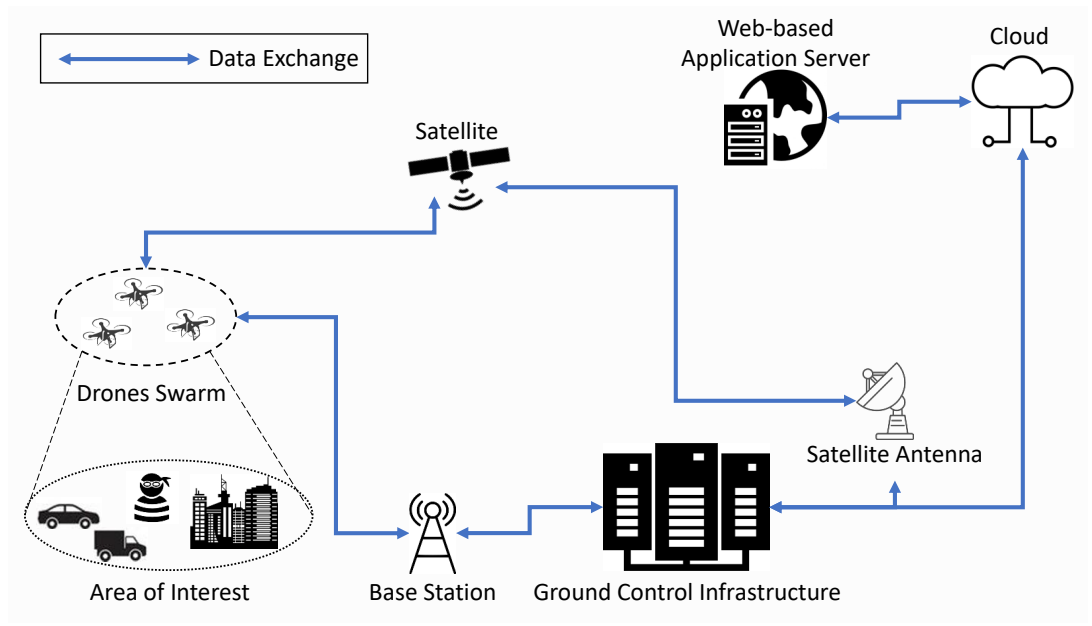

Figure 4: Reference IoD architecture.

The employment of drones has been extended in smart mechanism by integrating the Machine-to-Machine (M2M) communications. This enables drones to interact with sensors and service providers. Such a mechanism can be further evolved towards coordination schemes in between the sensor services with its upper layers, which is, in general, a fog and/or cloud environment. In the latter context, distributed intelligence and cooperation among logical nodes becomes of utmost importance to offload the elaboration provided by UAVs.

In Figure 4, the reference architecture for the IoD is depicted. In it, a swarm of UAVs is flying over an area of interest. While on flight, drones are communicating to the to a Ground Control Infrastructure leveraging a certain number of BSs. As suggested by the preliminary studies on $6 \mathrm{G}$ technology, drones are communicat- 
ing with satellites [10, 30, 24, 31, 32, 33, 34]. In particular, satellites are mainly communicating to drones to be activated and/or remotely controlled. These control messages are sent by the ground control infrastructure via satellite antennas. The whole set of information regarding the mission plans, the achieved mission goals and, eventually, remote control are handled by web-based applications in a distributed fashion leveraging cloud computing. In order to enhance the data transmission rate and the system capacity, 6G network has led a key role in pioneering the introduction of system intelligence using Artificial Intelligence (AI) [32].

As clearly comes out from this description, the IoD architecture must be considered as in a general-purpose fashion. In particular, the main claim of the IoD is that the network architecture is extremely versatile and able to meet several requirements at the same time. To clarify this, some of the main references of the present work are reported in Table 2 , where the application fields are classified together with the roles the drones are covering [10]. A detailed discussion of these points will be given in the remainder of the present work.

\subsection{IoD Economics}

Beyond the novelty, and possible innovation, that drones may lead in the wide context of civil applications, UAVs represent a promising perspective in several industrial segments [35, 36, 37, 38, 4]. The economic impacts associated to the wide employment and integration of UAVs is certainly going to have impacts on job creations. At the same time, industries will be able to cut costs from more effective means of transportation and distribution. The applications may vary for several reasons. First of all, the ever increasing technological readiness of drones is continuously increasing in terms of performances. For instance, drones are able to fly regardless of the payload: in fact, at the time being, UAVs may be equipped with cameras (e.g., photo or video), sensors, and radars. As for flight time and autonomy, drones are more and more able to fly for longer periods of time, thanks to enhanced battery and energy supply systems.

The work presented in [39] discusses the possible economic impact of the introduction of drones in multiple applications. Starting from what's stated in [40], and further confirmed in [41, 42, 37], the employment of drones at scale would bring $\$ 80$ billion by 2025 . This is motivated by the fact that a plenty of interesting functionalities that drones may expose when employed [43], such as improving public support given their intrinsic autonomy. Moreover, security can be enhanced according to the fact that social and economic impacts can be the result of the fact that surveillance or search and rescue activities can be carried out almost on their 
own by the drones. Another interesting contribution is [44], in which the main focus is on smart agriculture and smart farming technologies. This work discusses the details about precision application technologies, which implies the need for technologies able to work with variable-rate. The rationale for this variability may be application dependent and context dependent. For example, data acquisition related to crop monitoring when diseases or irrigation problems are detected may lead to sudden intervention, thus saving entire production sectors. At the same time, precision irrigation and weeding has been widely recognized as one of the leading pillar of smart farming. Irrigating on demand and constant monitoring of soil humidity Can be enabled by the analysis of aerial images. [45] provides similar forecasts, but in the UK market. Even though regulations and production sectors are different from other countries, what this study has in common with others is the fact that they share the same opinions in terms of the drones' role. Defined as game-changer, drones are expected to improve industrial processes at scale by $£ 42$ billion by 2030 . The study discuss is the role of technology in saving lives in harsh environments and in the case of hard working condition, providing concrete examples in case of utilities and oil and gas plants. The detailed analysis carried out in this contribution envisions several strategic fields in which the impact of drum is estimated to be of importance. in particular:

- Public and Defense, Health, technology.

- Technology, media and telecommunications.

- Construction and manufacturing

- Financial, insurance, professional and administrative services.

- Transport and logistics.

- Agriculture, mining, gas and electricity.

In a nutshell, drones may have direct impact on [36]:

- Consumer applications, thus including individuals, non-commercial and nonprofessional. A forecast on drones shipment for this sector indicates a total of 29 million units by 2021 .

- for enterprise drones, Business Insider Intelligence expects shipments to reach 805,000 in 2021 with a five-year CAGR of 51\% from 102,600 in 2016. 
- Government drones fall into two categories: military and public safety. Among the two, the former market can be considered as the most mature. The U.S. military has been using drones for combat since 2001, and it rapidly expanded its drone fleet to more than 7,000 by 2012 . The Department of Defense budget in 2016 allocated $\$ 2.9$ billion for more than 50 new drones for combat and surveillance, according to the Bard Center for the Study of Drones.

Industrial players may significantly cut costs and/or improve operations for the enterprises that will introduce or adopt them. As for the industrial applications that will mostly benefit from the introduction of drones, the most significant impacts will be on [36]:

- Infrastructure (45.2B \$).

- Agriculture (32B \$).

- Security (10B \$).

- Media and Entertainment (8.8B \$).

- Telecommunications (6.3B \$).

As for delivery, is has been estimated that there could be an increment on transportation speed $(\sim 50 \%)$, a positive effect on environmental impact (lower by almost 50\%), increased control over package delivery flow ( 40\%), and safety $(\sim 30 \%)$ [38]. Service provisioning may be related to systems and methods for delivering mail and goods using UAVs.

To sum up, the number and kind of fields that may benefit from the employment of drones are so numerous that their potential impact in automating and optimizing processes can only be roughly foreseen. At the same time, as happens every time a groundbreaking technology becomes available, massive drones employment may radically change every business not only in terms of time-to-market for products but, even more, in terms of process design and optimization.

\section{Physical Layer}

This Section discusses all the technological aspects and research challenges connected to the Physical Layer (PHY). In particular, the main topics of interests are: (i) Modulation techniques, (ii) Connectivity, (iii) Throughput maximization, 
(iv) Channel modeling and characterization, and (v) Communication technologies. The Section closes up highlighting the lessons learnt on this theme. The overall organization of this section is reported in Figure 5.

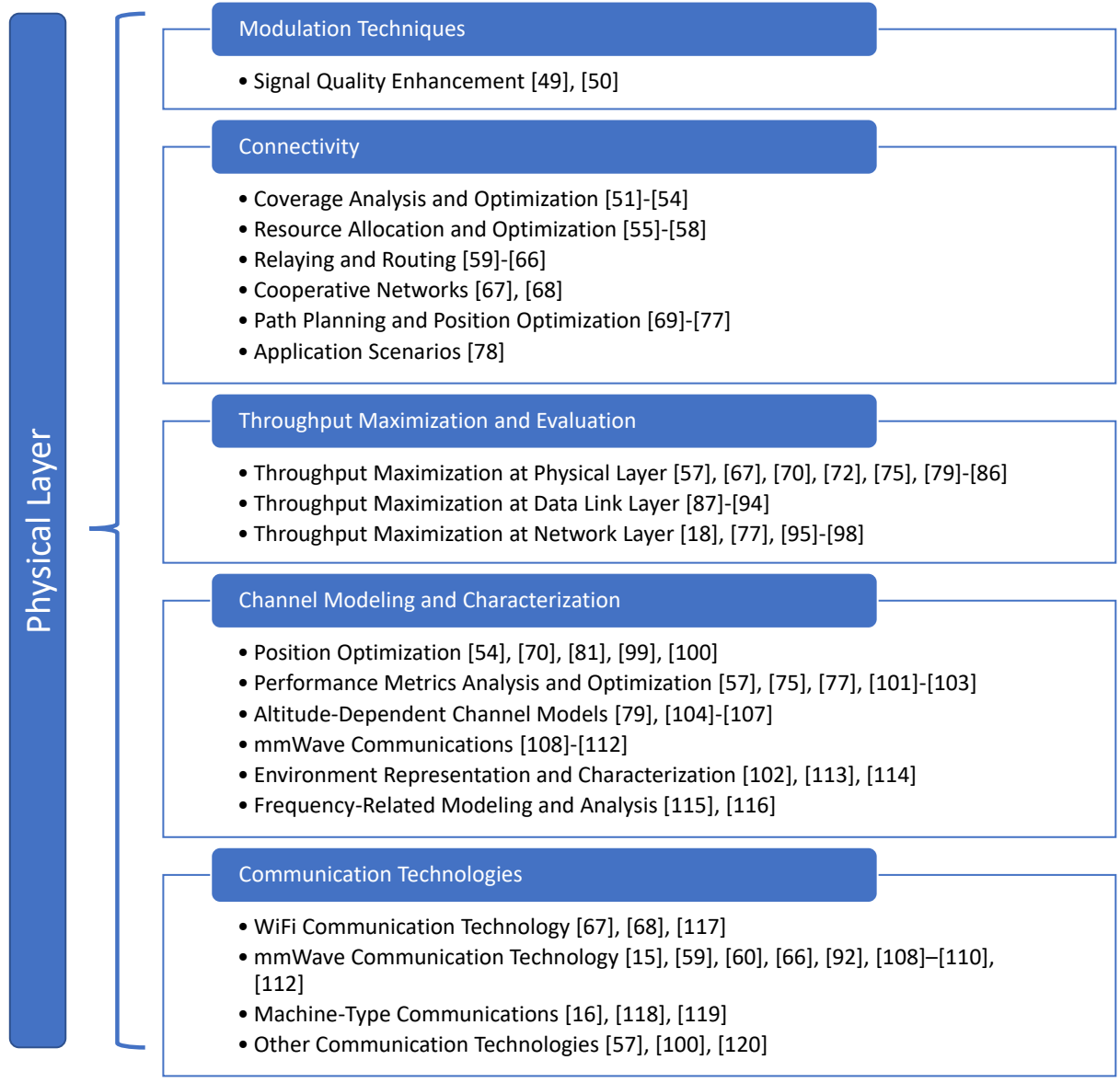

Figure 5: Physical Layer Taxonomy.

\subsection{Modulation Techniques}

There are some papers that analyze modulation techniques in the context of UAV communications [46, 47, 48, 49, 50]. They are discussed in the following subsections. 


\subsubsection{Signal Quality Enhancement}

More strictly related to the analysis of modulation schemes are the contributions [49, 50]. A modulation technique that increases the efficiency of the data link between UAVs is investigated in [49]. The analyzed modulation scheme is the Single Carrier - Frequency Division Multiplexing (SC-FDM), with the important goal to fulfill long-range communication and long battery life requirements for networks of UAVs. This scheme is already known in uplink communications for Long-Term Evolution (LTE) systems, but in this study is contextualized to UAV communication, by comparing its performance with the classical Orthogonal Frequency-Division Multiplexing (OFDM) modulation scheme. Performance analysis is carried out in terms of transmission power optimization, bit error rate and pulse shaping. An analysis of the OFDM modulation is considered in [50], to propose a noise suppression method for UAV communications. The proposed method filters electromagnetic impulse noise at receiving side; then it performs OFDM demodulation to suppress noise. The problem is tackled analytically, using models for the various electromagnetic pulses to analyze their influence on the OFDM-based communication. The electromagnetic pulse suppression strategy is then presented; it relies on a module that performs the separation of noise from the signal before OFDM demodulation, that acts in combination with a dual antenna method to reduce the bit error rate and increase the quality of the received pictures.

\subsection{Connectivity}

Connectivity is a topic widely analyzed in literature, as testified by the works $[51,52,53,54,55,56,57,58,59,60,61,62,63,64,65,66,67,68,69,70,71,72$, 73, 74, 75, 76, 77, 78]. They are mainly focused on: (i) analysis and optimization of coverage and resource allocation, (ii) relaying and routing, (iii) cooperative networks, (iv) path planning and position optimization and (v) connectivity issues in specific application scenarios, as described in what follows.

\subsubsection{Coverage Analysis and Optimization}

A connectivity analysis can be fruitfully exploited to study coverage performance of networks of drones in different contexts; this is the approach followed by the works [51, 52, 53, 54]. The work [51] proposes a coverage analysis that takes into account connectivity between UAVs and ground users. In this work, UAVs at low altitudes provide services to users falling into a coverage area whose shape is influenced by the UAVs directional antennas. The coverage analysis is carried out statistically as a function of the main UAV parameters, among which, the 


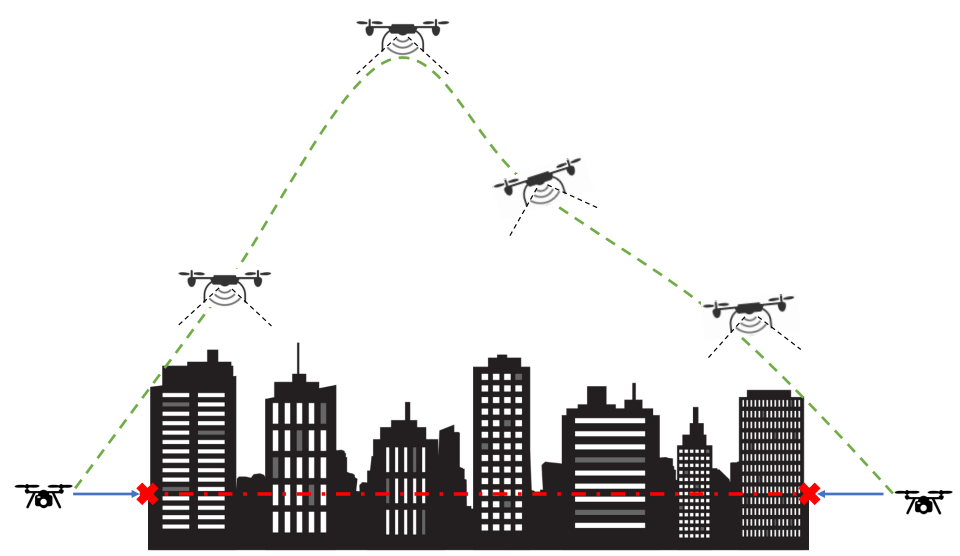

Figure 6: Non-Line of Sight (NLoS) communications enabled by drones.

Line of Sight (LoS) connection and LoS and NLoS (as graphically represented in Figure 6) interference between UAVs and ground users. A similar approach is followed in [52], where a coverage probability analysis is carried out in a multi-UAV scenario. In particular, here UAVs provide service to a ground receiver. The analytical expression takes into account both LoS and NLoS components, and aims at carefully capturing the influence of the dominant interferer on the receiver, with respect to the other interference contributions from the rest of UAVs. Another coverage study in a network that includes UAVs with relay functionalities is developed in [53], where the connection probability is studied in conjunction with coverage capabilities, for different types of traffic. LoS and NLoS connections are modeled statistically also in [54], to study the coverage performance of small cells of drones providing wireless access to ground users. The probability of LoS and NLoS connections are used to analyze the drones altitudes that maximize the coverage extension and minimize the transmission power.

Even if drones connectivity can be considered as a peculiar aspect of the physical layer, it also impacts on other layers of the protocol stack. For this reason, studies on connectivity are carried out also in several studies proposing strategies for resource allocation and optimization, relaying and routing, cooperative networks, path and position optimization, and analyzing specific application scenarios.

\subsubsection{Resource Allocation and Optimization}

The works [55, 56, 57, 58] take into account connectivity in the framework of resource allocation and optimization strategies. In these works, the probabilities 
of LoS and NLoS connections are used to describe theoretically Air-to-Air (A2A) and/or Air-to-Ground (A2G) links. Several factors are considered to model the link connectivity, like the environment, placement and density of buildings, and relative positions of UAVs and users. In [55] the model is exploited to develop a resource allocation algorithm in a cache-enabled UAV-based network, that optimally allocates the spectrum bands so that the queue stability of users and their bandwidth requirements are satisfied. In [56] the link connectivity model is exploited to maximize the average number of bits received by ground users, subject to constraints on the UAVs maximum flight times and with fair resource allocation guarantees. In the work [57] both LoS and NLoS path loss models are exploited to implement an algorithm that increases the throughput of a public safety network which leverages, at the same time, the unlicensed LTE spectrum of UAVs and the Wi-Fi spectrum of ground access points. The goal is to balance the load on the basis of the link quality of users.

\subsubsection{Relaying and Routing}

In the works [59][60][61][62][63][64][65][66] connectivity is analyzed in the context of relaying and routing strategies. The work [59] proposes a solution to increase the connectivity among devices adopting mmWave communication, through mobile relaying. To this end, the optimal position of the mobile nodes is found so that the link quality is maximized and the communication range is increased accordingly. In the work [60] the loss of connectivity is analyzed to justify the importance of an accurate prediction of node movements for an efficient design of the routing strategy. The proposed routing algorithm includes a feature that predicts the movements of the mobile node, to increase the message delivery ratio and reduce delay in case of short-lived connectivity. The work [61] models the connectivity between two nodes, in terms of expected connection time, to build a routing protocol that dynamically updates the routing path based on the prediction of the connection times of drones. In [62] a route recovery strategy is proposed, that exploits UAVs as relays interconnecting terrestrial networks in disaster scenarios. The route recovery over damaged networks is based on a topology discovery algorithm that identifies clusters of nodes, and the relative cluster heads, depending on their connectivity capabilities. In the routing protocol developed in [63] the most connected path is found to route data between vehicles of a VANET and UAVs assisting them. The path depends on the traffic density and the degree of connectivity on the road. An extension of the same protocol developed in [63] is proposed in [64]. It considers both routing between UAVs and vehicles and routing among UAVs. The UAVs connectivity is a requirement of the 
layered network architecture presented in [65]. The routing algorithm proposed in this work aims at minimizing latency while maximizing the Packet Delivery Ratio (PDR). The connectivity information is exploited to improve the routing performance. A connectivity model is developed in [66] to analytically describe the channel conditions of the back-haul links in 5G cellular networks that exploit the mmWave spectrum. The dynamic blockage of back-haul links is modeled in a network with UAVs acting as relay nodes. This aspect is part of a more complex analytical framework, that captures also the mmWave propagation characteristics and the UAVs mobility, to dynamically reroute data depending on the variable channel conditions.

\subsubsection{Cooperative Networks}

The works [67, 68] analyze connectivity among drones in cooperative networks. In particular, [67] proposes a distance control algorithm that finds the optimum distance among drones that cooperate for data transfer tasks, to guarantee the stability of the wireless connection and, consequently, of drones communication. The continuity of the connectivity is the main focus of the work [68], that proposes a network infrastructure, composed by drones that exchange data with ground users to manage critical events. The goal is to guarantee the service continuity by keeping the connectivity among UAVs in case of nodes replacement. To this end, a modification of the Optimized Link State Routing Protocol (OLSR) protocol, already known in literature, is proposed.

\subsubsection{Path Planning and Position Optimization}

There are several works that analyze connectivity to develop path planning and position optimization strategies $[69,70,71,72,73,74,75,76,77]$. The work [69] proposes the use of flying back-haul hubs to extend the coverage area in a $5 \mathrm{G}$ network. The hubs optimal location is wisely established, by solving an optimization problem, to provide connectivity between the core network and the ground BSs. Also the algorithm proposed in [70] aims at finding the optimal position of UAVs used as BSs to provide connectivity to ground users. The algorithm makes use of a theoretical channel model that describes the connectivity of the A2G links as a function of the propagation paths and the main features of the antenna arrays. Another path planning algorithm that takes into account connectivity can be found in [71]. This work studies the connectivity of a wireless network of balloons with UAVs acting as relays. The connectivity requirement is expressed as a function of the probability of the link outage among balloons. Depending on this, the proposed path planning algorithm finds the optimal UAVs positions that maximize 
the network connectivity. A path planning and resource allocation scheme that models connectivity in cellular-connected UAVs is found in [72]. In this work, analytical expressions of connectivity are used to model the interference level of the UAVs on the ground network and compute the optimal paths of UAVs that minimize it, together with the transmission delay and the time the UAVs employ to reach their destination. An approach that takes into account connectivity of A2A and A2G links is found in [73], where connectivity constraints are introduced to study the path planning problem for an aerial sensor network connected to ground Long-Term Evolution Base Stations (LTE-BSs). The connectivity constraints are expressed in terms of the outage probability of the link, which depends on the relative distance between aerial and ground nodes through A2A and A2G links. Based on this, the connectivity constraint is derived, which depends on the minimum link capacity. The work [74] develops a mobility model of a fleet of UAVs, where each UAV must keep the connectivity with its neighbor, taking also into account residual energy level and area coverage as decision criteria for the path planning procedure. In [75] the LoS probability is used to derive the coverage probability of an UAV providing service to ground users. The approach is similar to what developed in [52], but in this case the coverage probability expression is used to compute the optimal UAV position that maximizes the coverage area and coverage lifetime. The work [76] proposes a connectivity model between UAVs and ground nodes. Connectivity is modeled in terms of the outage probability of the terrestrial-aerial communication link, and the optimal UAV height that maximizes the coverage area in presence of fading is found accordingly. In [77] the probabilities of LoS and NLoS connections are used for a theoretical model of A2A and/or A2G links. This model is used to design a network formation algorithm that guarantees the connectivity between each UAV of the aerial network and a gateway node through at most one path.

\subsubsection{Application Scenarios}

The approach proposed in [78] differs from all the others described above, since the link connectivity is related to the users QoE. Specifically, the LoS connectivity, derived in terms of probability of connection loss, is exploited to model the transmission links in a network of UAVs. The goal is the optimization of the users QoE, that in this case depends on the user data rate, delay, and device type.

\subsection{Throughput Maximization and Evaluation}

This section describes all the works proposing strategies for throughput optimization and evaluation, in different IoD scenarios and at different layers of the 
protocol stack (physical, data link and network layer) [67, 79, 80, 70, 81, 72, 82, 83, 84, 85, 86, 57, 75, 87, 88, 89, 90, 91, 92, 93, 94, 95, 96, 18, 97, 77, 98].

\subsubsection{Throughput Maximization at Physical Layer}

As expected, the majority of the works on throughput maximization and evaluation are focused on the physical layer of the protocol stack [67, 79, 80, 70, 81, 72, 82, 83, 84, 85, 86, 57, 75.

Some of the most significant contributions of the theme deal with position and path optimization strategies of UAVs [67, 79, 80, 70, 81, 72, 82, 83]. More specifically, in [67] throughput performance is studied to show the effectiveness of a distance control algorithm, with the goal of guaranteeing the stability of the wireless connection in $\mathrm{A} 2 \mathrm{G}$ links in case of drone replacement in the network. The proposed algorithm dynamically changes the drones position so that a minimum throughput requirement is satisfied. The work [79] proposes a strategy for throughput maximization in a UAV-based wireless network where a single UAV is used as BS to serve ground users. The proposed strategy optimizes jointly the UAV path and the scheduling times of users communications. The solution of the optimization problem aims at maximizing the minimum throughput over all users, guaranteeing fairness among them. The same network scheme of UAVaided cellular network is proposed in [80]. In this architecture, the UAV offloads the ground BS and serves the UEs at cell edge. The goal of this scheme is the maximization of the minimum throughput of the UEs, by jointly optimizing the UAV trajectory, resource allocation and users partitioning between the UAV and the terrestrial BS. The issue of aggregate data rate maximization in the DownLink (DL) channel is tackled in [70]. In this work, an approach is proposed that optimizes the hovering locations of UAVs acting as flying BSs to maximize the DL aggregate rate, exploiting UAVs mobility and beam-forming techniques. This goal is obtained through a search algorithm, that finds the hovering locations with a low interference leakage, that maximize the Signal-to-Noise Ratio (SNR) of the ground users. The work [81] considers a wireless network where UAVs act as relay. In this scenario, an algorithm is proposed that exploits the LoS information to find the optimal UAV positions that maximize the end-to-end throughput. These positions are found by means of a model that takes into account the signal propagation properties of the $\mathrm{A} 2 \mathrm{G}$ channel. The computational complexity of the proposed algorithm is shown to be low, if compared to other UAV algorithms of position optimization. The work [72] proposes a path planning algorithm for a network of cellular-connected UAVs. The problem is modeled as a dynamic game among UAVs, in which each UAV learns the optimal path and the trans- 
mission power along the path, that optimizes the latency and the rate per ground user. To this end, the achievable data rate per user is modeled as a function of the channel characteristics, and the UAV path is planned so that the interference of UAVs on the ground network and the transmission latency are both minimized. Allocation of resource blocks assigned to each user is managed accordingly, so that the per-user data rate is increased. In the work [82] an algorithm is proposed that optimizes the direction and distance of an UAV acting as a BS, depending on the amount of randomly moving users in a sector of the served cell. The users throughput is derived as a function of the SNR; the optimal UAV position is then computed so that the average throughput is maximized in the cell. The goal of the work [83] is the throughput maximization in a multihop UAV network. The network model proposed takes into account parameters related to the observation area and signal attenuation due to obstacles. These parameters are exploited to derive an analytical expression of the upper bound of the throughput. The optimal locations of UAVs that maximize this bound are found accordingly.

Some papers analyze throughput for the optimization of spectrum efficiency [84, 85]. More specifically, in [84] an optimization algorithm is proposed that takes into account data rate. The algorithm aims at maximizing the spectrum efficiency and energy saving of an UAV that relays data between two Ground Stations (GSs), by jointly optimizing the time allocation of data to be received and forwarded, and the UAV speed and trajectory. The expression of the maximum instantaneous throughput is derived for the links between the GSs and the UAV and is used to maximize the spectral and energy efficiency. An approach for energy efficiency and throughput improvement can be found in [85]. In this work, a study on the adaptive modulation is carried out for networks of Unmanned Aircraft Systems (UASs) collecting data from some sensor nodes. Based on the analysis of the impact of the UAS trajectory on the modulation scheme, a problem is formulated that maximizes the throughput per energy of UASs, while preserving fairness among transmissions between sensor nodes and UASs. The solution proposed exploits an approach based on the game theory.

An analysis on the impact of the link quality on system throughput is found in the works [86, 57]. In [57], a study is carried out on the use of unlicensed spectrum for UAV-BSs communications in a heterogeneous network with Wi-Fi ground Allocation Policys (APs), to investigate the enhancement of achievable throughput in emergency situations. To this end, a game theoretic approach is proposed, and the related algorithm configures UAV-BSs transmissions to balance the load between UAV-BSs and ground APs. The solution found takes into account the users link quality and the loads of UAV-BSs and APs, aiming to ensure 
a satisfactory throughput for all the users. The work [86] studies the impact of the characteristics of the wireless link on the system throughput in a UAV-toground link. Several parameters are chosen, i.e., antenna orientation, UAV height, yaw and distance between the UAV and the ground access point, for experimental evaluation of throughput performance.

The paper [75] analyzes rate and coverage performance for UAV-based communication with Device-to-Device (D2D) links. To this end, an analytical model for the system sum-rate is first derived as a function of the UAV height and number of users, in a scenario of a single static UAV transmitting data to a D2D network. Rate performance are derived as a function of Signal to Interference-plus-Noise Ratio (SINR), UAV altitude and density of D2D links, also showing the existence of an optimal UAV altitude and a users density that maximize the rate.

\subsubsection{Throughput Maximization at Data Link Layer}

Several works analyze throughput at link layer, even though the discussion of the proposal is carried out at physical layer [87, 88, 89, 90, 91, 92, 93, 94].

In some papers, the throughput analysis is carried out in the framework of VLC systems [87, 88, 89, 90]. In [87], an analytical model is developed for the Carrier Sense Multiple Access with Collision Avoidance (CSMA/CA) protocol. It considers the relationship between the physical and MAC layers of the protocol stack. In this context, the paper shows that the multi-packet reception capability of the VLC system, i.e., the capability to decode multiple packets coded with orthogonal sequences, reduces packets collisions and increases the number of simultaneous transmissions, also increasing throughput. A throughput evaluation is carried out in [88]. The goal of this paper is to develop a IEEE 802.15.7 simulation module for ns-2, based on an accurate modeling of the physical layer and of the CSMA/CA protocol at MAC layer. The mean throughput of a system composed by a coordinator servicing 12 nodes is derived, to validate ns- 2 simulation results and compare the module performance with a Markov-based analytical model present in literature. A theoretical study of the IEEE 802.15.7 standard that takes into account throughput is found in [89]. The study is conducted ad MAC layer, through theoretical analysis and computer simulations. More specifically, a Markov chain is used to model the MAC layer with the use of the beacon frame and CSMA/CA. In this context, closed-form expressions are derived for the main performance metrics, including throughput. The model is then validated through simulation. Also the work [90] evaluates throughput performance at MAC layer in a VLC system where two nodes are in reciprocal Field-of-View (FoV), and one of them continuously transmits data to the other. The MAC layer throughput is 
evaluated as a function of the frame payload (at a fixed distance) and the nodes distance (with a fixed frame payload). Throughput evaluations are carried out in two configuration scenarios, one-hop (direct communication between two nodes) and two-hop (three nodes, with one node forwarding packets at network layer between the other two).

Access schemes at MAC layer for throughput improvement are discussed in the works [91, 92]. A radio access scheme thought for multi-UAV networks with relaying capabilities is proposed in [91], to increase throughput. This work proposes a modification of the classical Long-Term Evolution-Advanced (LTE-A) frame structure, together with a scheduling algorithm that dynamically changes the ratio of DL and UpLink (UL) subframes, to increase the efficiency of relay communication and, thus, the network throughput, especially in cases of longer distances. The work [92] discusses the integration of the mmWave communication in last-generation UAV-based cellular networks. This work mainly focuses on the design of a multiple access scheme that, in combination with multiplexing techniques and beam-width selection strategies, improves the system throughput in presence of concurrent transmissions.

Resource allocation schemes for throughput optimization are discussed in [93, 94]. In [93], a priority-based frame selection scheme is proposed, to optimize throughput and energy saving. This scheme can be exploited to improve the efficiency of data delivery in a sensor network that adopts a single UAV. The framework comprises the frame selection scheme, another scheme that adjusts the CSMA/CA contention window at MAC layer, and a routing protocol that selectively transmits frames based on their priority and the relative distance between nodes. A throughput-aware resource allocation algorithm is proposed in [94]. The model is suitable for data transmission between an UAV and GSs. In this work, the effective throughput is used as requirement to optimally allocate communication resources, expressed as time slots, for communication between the UAV and GSs. Throughput is computed as a function of different parameters, i.e., allocated time slots, modulation scheme and coding rate. The model aims at increasing the minimum throughput, as derived from the solution of the optimization problem.

\subsubsection{Throughput Maximization at Network Layer}

Throughput is also considered in some papers discussing issues at network layer [95, 96, 18, 97, 77, 98].

A couple of works focus on relay schemes for throughput improvement [95, 96]. In [95], throughput maximization is discussed for multi-hop networks with clusters of relays, as illustrated in Fig. 7. The goal of this work is to find the opti- 


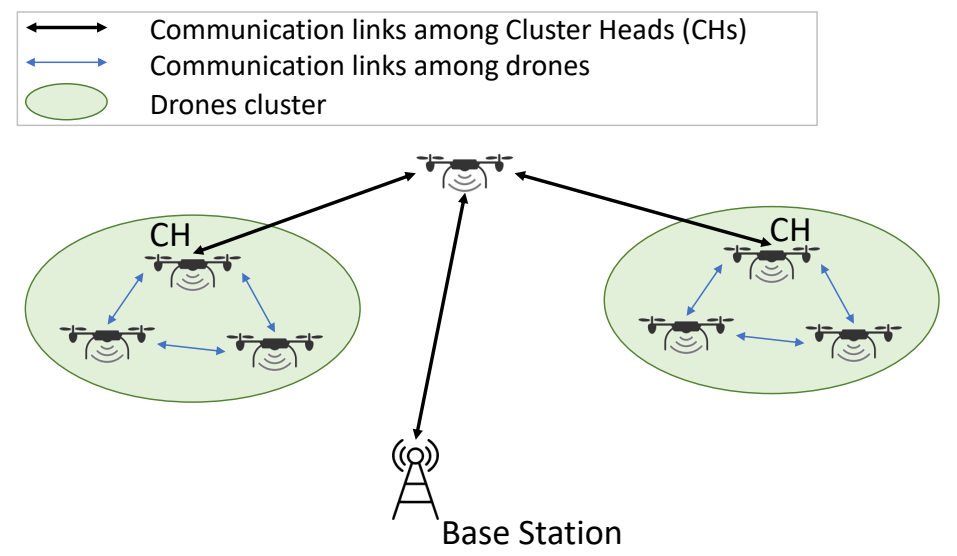

Figure 7: Communications among clusters/drones swarms and Base Station thanks to relay node.

mal deployment of relay nodes, in terms of number of hops and clusters locations, that minimize the outage probability, together with the optimal number of relays that maximizes the throughput.

The work [96] proposes a relaying scheme that schedules transmission so that the energy consumption is minimized, at the same time maximizing the network throughput in presence of bit errors. To this end, an optimization algorithm is proposed, together with a simplification that provides a sub-optimal scheduling solution in a much faster way, at the same time guaranteeing throughput improvements.

Novel routing schemes that take into account throughput are developed in [18, 97]. A geographical routing (also referred to as geo-routing) scheme is implemented in [18]. The solution is known by literature and adopted in UAV-based systems. Its goal, if compared to the classical implementation, is to increase throughput and decrease delay. The core of the proposal is a modification of the frame structure at physical layer of the geo-routing scheme with contention-free capabilities, that reduces overhead, thus reducing delay and increasing throughput. A throughput stability analysis is found in the work [97]. This work proposes a centralized routing protocol for a fleet of UAVs, that exploits the information coming from a ground control system to keep connectivity among drones and throughput stability, while shortening the route update times.

Network architectures that take into account throughput are found in [77, 98]. The proposal of the work [77] is a scheme in which UAVs act as a flying backhaul network connecting ground BSs. The flying network is dynamically formed 
by choosing the $\mathrm{A} 2 \mathrm{~A}$ and $\mathrm{A} 2 \mathrm{G}$ links and the network topology that maximize a given utility function, through a network formation game approach. The chosen utility function considers the achievable data rate, the number of relayed packets, and delay in both DL and UL directions. A hierarchical network structure is proposed in the work [98], that provides a stable throughput and extends the network lifetime. It is composed by satellites, airships and UAVs. Based on an estimation of the weather conditions, a topology control and routing algorithm are proposed, that distribute the traffic load among the network nodes so that bottlenecks of link capacity can be avoided. In this way, an acceptable performance in terms of network lifetime and average throughput is guaranteed, for different weather conditions.

\subsection{Channel Modeling and Characterization}

This section describes all the works related to channel modeling and its analytical description together with the most relevant techniques for characterizing the communication medium in use by drones $[99,54,100,70,81,75,101,77,57,102$, 103, 104, 76, 105, 106, 107, 108, 109, 110, 111, 112, 113, 102, 114, 115, 116]. Different models are presented aiming at mathematically describe several aspects of IoD communication scenario, that are described in what follows.

\subsubsection{Position Optimization}

Some works analyze the communication channel for UAV position optimization [99, 54, 100, 70, 81]. Specifically, the works [99, 54] refer to the A2G channel. An A2G channel model is employed in [99] in a network of UAV-BSs. This work proposes a position optimization algorithm in the 3D space, that minimizes the UAV lifetime. In this scenario, the A2G channel model is used to compute the average path loss as a function of the environment, the carrier frequency and the type of the link (LoS or NLoS). This model is exploited to find the optimal UAV hovering altitude that minimizes the transmission power, and that contributes to find the optimal position of UAVs in the 3-Dimensional space. A channel model is presented in [54] to study small cells of UAVs acting as aerial BSs. More specifically, the A2G channel model is developed, including LoS and NLoS paths and the related path loss. The probabilities of LoS and NLoS connections are derived. Further, those values are characterized in terms of average path loss, which depends on the UAV height and coverage radius. This channel model is exploited to find the optimal UAV altitude that maximizes the ground coverage area, and study the coverage performance of UAV-BSs. A channel model is adopted in [100] to propose a solution that minimizes the energy of micro-UAVs in cognitive radio 
systems. The goal of this work is to find the optimal drone position and power level that minimize the UAVs energy consumption. To this end, the path loss and channel gain are analytically derived, to model the A2G and Ground-to-Ground (G2G) channels. These models are then exploited to solve the joint optimization problem of power allocation and drone positioning. A narrow band channel model is introduced in [70] to study the optimal hovering locations of UAV-BSs to provide wireless connectivity during time-limited events. The model used for the wireless link is expressed as the sum of different propagation paths with different gains from UAVs to users. This model is then used to find the optimal UAVs positions that maximize the user SNR. To this end, an antenna array is adopted; this solution is of interest since it introduces the contribution of beam-forming to the SNR improvement and locations optimization. The same approach of [70] is used in [81], where channel models are exploited to find the optimal UAV position that maximizes the end-to-end throughput, but without knowing the whole radio map of the signal strength. To this end, two different channel models are employed: an A2G channel model between the BS and the UAV that exploits the only LoS propagation, and the channel between the UAV and the user, that instead models the path loss exploiting both LoS and NLoS propagation.

\subsubsection{Performance Metrics Analysis and Optimization}

Channel modeling is tackled for analysis and optimization of other performance metrics in [75, 101, 77, 57, 102, 103]. In [75, 101], the A2G channel is modeled to optimize coverage and rate performance in UAV networks. As known by literature, the model used in these works takes into account three different groups of signals: LoS, reflected NLoS, and multiple reflected signals that cause multipath fading. Similarly to several other works on the topic, LoS and NLoS links are modeled separately to derive the received signal power at users locations. Based on this procedure, the goal of [75] is to analyze the coverage and rate performance in a UAV-aided network in presence of D2D links, while the work [101] aims to derive the optimal coverage probability and deploy multiple UAV-BSs with directional antennas. Other theoretical approaches that model the A2G channel for analysis and optimization of performance metrics are found in [77, 57, 102]. The work [77] takes into account both A2A and A2G channel models to design a multi-hop backhaul network composed by UAVs. The A2G channel is modeled statistically, through LoS and NLoS links that take into account the higher attenuation due to diffraction and shadowing. The only LoS link is instead considered for the A2A links. These models are then exploited to evaluate the SINR of the A2G link and the SNR of the A2A link, to derive the 
achievable end-to-end rate and design the UAVs network formation accordingly. The same approach to the A2G channel modeling adopted in [77] is found also in [57], where the channel is modeled statistically, through LoS and NLoS path loss, as already done in the past literature. The goal of the work is to balance the load between UAVs and Wi-Fi ground APs during emergency situations, based on the users link quality. The path loss model is exploited to compute the received SINR that, together with the load balancing requirement, determines the best association between the user and the BS. Differently from the studies mentioned above, the work [86] focuses on $\mathrm{A} 2 \mathrm{G}$ channel measurements to characterize path loss exponents in the UL and DL channel, in two different environmental scenarios. Several parameters are considered in the field experiments: antenna orientation, UAV height, yaw and distance. The impact of these metrics on the Received Signal Strength (RSS) and throughput is evaluated. A channel model is adopted in [103], that proposes a strategy for optimization of resource allocation so that the transmission delay of packets in an UAV-based cellular network is minimized. Part of this study is dedicated to the communication channel model based on nonLoS paths with Rayleigh fading. This model is then used to derive the power received at destination node, that is exploited to derive the transmission rate first, and then the mean packet arrival rate and transmission delay, that are optimized by solving an optimization problem.

\subsubsection{Altitude-Dependent Channel Models}

Some works develop channel models that take into account the influence of UAVs altitude [104, 76, 105, 106, 107]. The works [104, 76] propose an A2G channel model to study the effect of the altitude of an UAV-BS on some performance parameters, i.e., power, capacity gain [104], path loss and scattering [76]. The optimal UAV height is found that brings to the best trade-off between the optimal values of these parameters, through an extension of the A2G channel model that takes into account the UAV height in path loss and fading. The validity of the proposed model is tested also for low-altitudes UAVs. The work [105] focuses on the analysis of A2A channel features. Its goal is to extend the Rice model, which is usually involved in theoretical characterization of the A2A channel. Such studies are usually conducted by varying the UAV altitude, to provide a mode accurate description of the multipath effect. Based on real RSS measurements, an estimation of the main Rice model parameters that describe the fading and multipath effects is derived, in dependence of the UAVs altitude. The Rice channel model is extended accordingly. Modeling of the propagation channel for low-altitude UAVs is the goal of [106]. The UAV communication channel is studied through 
narrow-band and broadband measurement campaigns carried out in a suburban area, capturing the main channel characteristics: path loss, fading, power delay profile, multipath components, and root-mean-square delay spread. This work carries out an analysis of the A2G channel characteristics for low UAVs heights. The work [107] is entirely focused on channel models. Its primary goal is to model the path loss exponents of the radio link between an UAV acting as UE, and the cellular network. The proposed model takes into account the UAV height, which is exploited to model both the path loss exponent and the shadowing effect. The model is validated in a real scenario, using a UAV connected to two different LTE networks.

\subsection{4. mmWave Communications}

Channel modeling and characterization are developed for mmWave communications in [108, 109, 110, 111, 112]. A characterization of the A2G channel is developed in [108] for UAV communication in the mmWave band. The goal of this work is to analyze the main characteristics of the A2G channel in different scenarios, each one with different kinds of mmWave propagation. Simulations are used to study the channel characteristics between a fixed ground station and a UAV that moves along a linear trajectory at a constant velocity, for different UAV heights. The most suitable model for the RSS behavior is derived accordingly. In the work [109] channel modeling is discussed for mmWave communication in UAV cellular networks. Channel characteristics are illustrated, discussing the most relevant issues of mmWave propagation, in terms of propagation loss, scattering, multipath components, and reflections around the UAVs. The most suitable channel model is then chosen to derive beam-forming vectors for different users. mmWave channel for DL transmission is studied in [110], in a network of UAVs connected to cellular BSs. The model takes into account the multipaths and their angle of departure, the height and horizontal distance of the UAVs from the BSs, the transmit antenna structure at the BS (composed by an antenna array), and the path loss of the DL channel. The LoS component of the paths is considered as predominant in this model, that is not suitable for low UAVs altitudes. The goal is the evaluation of energy efficiency in multiple access schemes for mmWave DL communications. The work [111] analyzes the coverage performance of cellular networks assisted by UAVs in mmWave communications. To this end, a channel model is introduced. It is designed as to exploit the only LoS component of the links between UAVs and mobile terminals. Due to the adoption of mmWave technology, the model takes into account the possibility that the LoS signal is blocked by obstacles. Also small scale fading and shadow fading effects are considered, 
through probability distributions. This model is exploited to define the strategy of user association and a cooperative clustering scheme for UAVs that maximize the coverage performance of the network. The characteristics of a mmWave channel in UAV communications with an antenna array are investigated in [112]. The channel is characterized through the Inverse Discrete Fourier Transform (IDFT) to obtain an analytical description of the channel behavior in the discrete time domain. The main parameters of this description are estimated to provide a channel tracking method that takes into account angle information, Doppler shift and UL/DL channel gains.

\subsubsection{Environment Representation and Characterization}

Some papers exploit channel models in specific environmental scenarios [113, 102, 114]. An algorithm to learn the channel characteristics between a UAV and a ground user is proposed in [113]. To this end, the radio channel is characterized through a propagation model based on a representation of obstacles whose configuration is stored into a map, together with other channel parameters. These data are used to estimate and reconstruct the channel propagation characteristics based on a reduced set of measurements, with relatively small prediction errors in the radio map reconstruction. In [102] an A2G channel model is described and used for UAV-BSs providing wireless service to ground users. As discussed in other works [77, 57], the probability of LoS link between the UAV and the user is evaluated, as a function of the environment and the horizontal and vertical distances between the UAV and the user. Based on this, the path loss is modeled and used to evaluate the conditions in which the ground user can be served by the UAV. A type of A2G channel model that takes into account detailed information (i.e., shapes, intersections and heights of buildings, reflection, diffraction, propagation mechanisms, etc.) is presented in [114]. Its main goal is to accurately characterize the path loss characteristics of the links between UAV-BSs and users, to derive a model suitable for altitudes both lower and higher that the buildings heights. The model parameters are optimized, based on the UAV altitude, so that the root-mean-squared-error is minimized.

\subsubsection{Frequency-Related Modeling and Analysis}

A couple of works deals with the specific problem of characterizing the channel in the frequency domain [115, 116]. The work [115] proposes a technique to estimate and compensate the Inter-Carrier Interference (ICI) for high speed UAVs. Based on the analytical expression of OFDM symbols, the channel matrix estimation is performed in the frequency domain, to accurately estimate the Doppler 
effect and the amplitude of all the paths. A classification framework that takes into account both spatial and spectral information is then adopted, to correctly evaluate the received data and the channel estimation and equalization method. A theoretical channel characterization is proposed in [116] to equalize channels that present selectivity in both time and frequency domains (the so-called doubleselective channels). In this work, doubly-selective channel equalization is studied for the transmission of continuous phase modulated signals, that usually present a high computational complexity. The goal is to present new equalization techniques that simplify the equalizers, even at a cost of approximations. To this end, the channel impulse response is characterized through a causal Finite Impulse Response (FIR) system.

\subsection{Communication Technologies}

The right choice of the wireless technology used for drones communication (LTE, Wi-Fi, VLC, WiMax, etc.) is of great importance to optimize the communication performance of networks of drones. It depends on many factors, including the type of the UAV tasks, their duration, the environment, drones mobility, communication range, limitations on transmitted data or energy consumption: technologies like cellular networks, WiMax and satellite communications guarantee a wide coverage area, high transmission bandwidth, high throughput and reliable connectivity, but are energy-consuming and often introduce high delays. Counterwise, technologies like Bluetooth and Zigbee have a low power consumption, data degradation, implementation costs and delays, but also a low transmission bandwidth, throughput, and are more subject to interferences caused by obstacles. All these aspects are covered in the works [117, 67, 68, 59, 110, 92, 60, 66, 108, 109, 112, 15, 16, 118, 119, 100, 57, 120].

\subsubsection{WiFi Communication Technology}

WiFi technology is explicitly addressed in the papers [117, 67, 68]. The work [117] mentions the Wi-Fi technology to build an ad-hoc network and implement a communication protocol for high-quality video transmission between drones sensing an area and mobile devices. The proposed protocol introduces an ad-hoc routing protocol that allows three drones to work together in the same network and expand the sensing area. Wi-Fi is used also in [67], to implement a distance control algorithm, that finds the optimal distance between two UAVs that maximize the stability of the wireless communication, and minimize the number of UAVs utilized to cover a given area. In [68] Wi-Fi is chosen as the most suitable technology for an air-to-ground communication network composed by UAVs and 


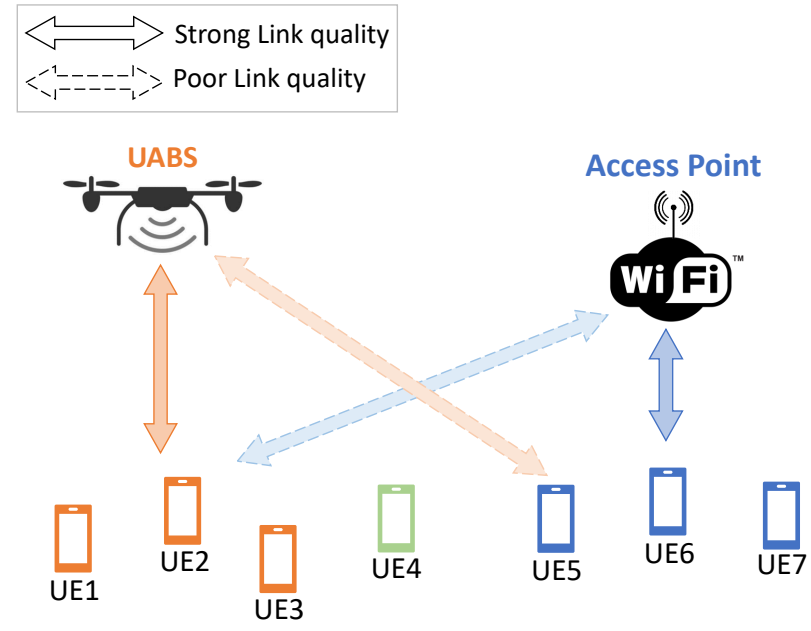

Figure 8: Cellular technology aided by an Unmanned Aerial Base Station (UABS) combined with Wi-Fi to provide connectivity to User Equipments (i.e., users).

ground users, as illustrated in Fig. 8. The goal of this study is to keep a service continuity when a UAV is replaced because of limited battery lifetime. The choice of Wi-Fi is motivated with its ease of deployment, the use of unlicensed spectrum, and the need to support applications with different vales for throughput in the same network.

\subsection{2. mmWave Communication Technology}

Several works analyze the mmWave technology for UAV-aided networks [59, 110, 92, 60, 66, 108, 109, 112, 15].

The mmWave technology is introduced in the work [59] to propose a strategy for an UAV to find automatically and accurately its optimal relay position, so that data can be forwarded in short time. mmWave communication advantages (very high frequency and increased bandwidth, short wavelength that allows beam-forming) and disadvantages (attenuation in free space propagation, blocking effect due to obstacles and objects) are described in detail, to justify the need of large use of relaying in mmWave communications, and introduce the proposed strategy.

The adoption of mmWave in UAV-aided cellular networks is investigated in [110, 92, 60]. In [110, 92] multiple access schemes are discussed for mmWave communications. The mmWave channel model is introduced in [110] to evaluate the energy efficiency of different multiple access schemes in a cellular-connected 
network that uses UAVs. The paper [92] proposes a multiple access scheme design for $5 \mathrm{G}$ mmWave UAV networks acting at both the physical and MAC layers, where concurrent transmissions are multiplexed into a single beam so that interference in the single beam is transformed into concurrent transmissions, and interference among adjacent beams is reduced. In [60], different aspects related to the mmWave technology are investigated: the channel propagation characteristics, the implementation of beam-forming techniques that account for channel variations, the impact of the Doppler effect due to UAVs mobility, and the adoption of spatial-division multiple access for mmWave to increase the network capacity. Some algorithms for optimization of UAVs mobility are also considered, to avoid signal blockage. Finally, the relationship between UAV positioning and user discovery is discussed, to analyze the reciprocal impact of one over the other.

The mmWave technology is exploited in [66] to model backhaul links in UAVassisted networks in urban environments, with UAVs acting as relay nodes. This work develops a mathematical model that takes into account the multipath propagation and the blockage effect of the mmWave backhaul link, and the mobility of both signal blockers (humans, objects, etc.) and UAVs. These phenomena are modeled accurately by using the New Radio technology, which is a backhaul architecture with reconfiguration capabilities that reroutes dynamically data to alternative paths, to increase connectivity and data reliability. The study of mmWave backhaul performance is conducted in both spatial and temporal domains.

The main goal of [108] is to characterize the mmWave A2G channel used by UAVs communications through simulations. Two mmWave bands are chosen for simulation, analyzing the RSS and Root Mean Square Delay Spread (RMS-DS) of multipath components (MPCs) for different UAV heights and environments (urban, suburban, rural, and over sea). Simulation results are also exploited to build a channel sounder.

Training and tracking strategies for mmWave UAV communications are presented in [109, 112]. The design of beam-forming codebooks with different beam widths is proposed in [109], that starts from the analysis of the channel propagation characteristics, to fasten the beam-forming training and tracking phases. The impact of the Doppler effect due to UAVs mobility is investigated, also carrying out an analysis of spatial-division multiple access schemes and signal blockage events. A channel tracking strategy is presented in [112]. Based on an analysis of the mmWave UAV channel with a beam squint effect, the proposed channel tracking method exploits the information on angle and Doppler reciprocity to obtain the channel state information exploiting only one pilot symbol, and reducing the feedback overhead. 


\subsubsection{Machine-Type Communications}

The works [16, 118, 119] analyze different technologies for Machine-Type Communication (MTC) in UAV networks. In [16] they are mentioned to discuss D2D communications in public safety applications scenarios. This work is mostly a review paper on the recent advances in D2D technology, and tackles the issues of device discovery, D2D clustering and relaying, and Vehicle-to-Vehicle (V2V) communications. In this context, together with the D2D technology as standardized by $3 \mathrm{GPP}$ rel.13, to increase coverage capabilities in critical situations, several other technologies are discussed, that allow D2D communication in unlicensed spectrum: Wi-Fi, Bluetooth, ZigBee, etc. [16]. All these technologies have also the advantages of high data rates, low latencies and implementation costs, and a wide adoption in mobile phones and devices. The paper [118] focuses on mission critical Machine-Type Communication (MTC) and investigates some connectivity options like D2D links and drone-assisted networks to satisfy the MTC requirements. Specifically, LTE technology is combined with Wi-Fi for D2D communications between mobile devices and UAVs, to increase connectivity and data reliability. In addition, the mmWave technology is studied in the scenario of small cells of drones that act as APs to increase the network coverage where the performance of the LTE network is poor. The technologies introduced in [119] are used to describe the main specifications of localization techniques in a M2M network composed, among others, by drones. The main goal of this paper is to propose a mathematical model that describes the structure of the M2M network that adopts agents for efficient localization of nodes.

\subsubsection{Other Communication Technologies}

Other communication technologies can be found in [100, 57, 120].

The Cognitive Radio (CR) technology is analyzed in [100] as a promising solution to mitigate the main issues in spectrum utilization of the other wireless technologies (Wi-Fi, Bluetooth, or cellular communications) for UAV-based applications. The CR technology allows to access the spectrum in an opportunistic way, by sensing the spectrum through advanced radio techniques, and to transmit data over the spectrum bands that are not utilized by other transmissions. It also allows utilizing simultaneously the same spectrum to serve different users, but without exceeding an interference threshold. This paper proposes an energyefficient optimization strategy, that finds that optimal UAV location and transmit power level so that a UAV can transmit data using the CR technology, while respecting the data rate threshold of the spectrum owner.

The work [57] investigates the possibility of using the Long-Term Evolution - 
Unlicensed (LTE-U) technology for communication among UAV-BSs, to increase throughput by exploiting the unlicensed spectrum. In critical scenarios, UAVBSs make use of LTE-U to fill coverage gaps due to the damaged infrastructure that adopts Wi-Fi. The goal of this hybrid infrastructure is to quickly deploy an on-the-fly cellular network able to fill the gap of throughput requirements of the damaged Wi-Fi ground network, through a game theoretic approach that selects the radio access technology technique that achieves load balancing among LTE-U UAV-BSs and Wi-Fi APs.

Optical wireless communication is studied in [120] for so-called Free Space Optical (FSO) communication systems, that exploit the optical signal for high data rate communications over relatively long distances. This paper focuses on FSO systems where UAVs act as relays. The study is conducted by assuming that relays are buffer-aided, and they can move in the space (i.e., they are not stationary), and evaluating the impact of these two assumptions on the FSO system performance. Two application scenarios are also discussed and validated through simulation by taking into account the outage probability as performance metric.

\subsection{Comparison with Other Surveys}

Several topics related to the physical layer are discussed in the surveys [12, 3, 5, 11, 8, 15]. Connectivity is discussed in [12, 3]. Nevertheless, in both these works its analysis is only related to coverage strategies and in some specific application scenarios or network architectures. Some works on throughput maximization and evaluation are mentioned in [3, 5, 11]. The related analysis mostly refers to routing strategies, even if some insights are provided also for hybrid network architectures [5, 11]. A throughput analysis is also provided in [8, 5] but without analyzing novel strategies for its optimization. Channel modeling and characterization is partially discussed in [5, 15]. Nevertheless, in the survey [5] the channel analysis and modeling is tightly bound to measurement systems for the A2G links of low-altitude UAV networks and platforms. Similarly, in the review work [15] channel characterization is studied with the only reference to the mmWave technology, pointing out the most significant challenges and opportunities related to this topic. Together with the work [15], also the survey [11] analyzes and classifies in detail the most widely used communication technologies for IoD scenarios. The drawback of the approach followed in [11] is that this survey neglects other newer technologies, such as mmWave or VLC.

If compared to the surveys mentioned above, the value added of this survey is to enhance and complete the analysis on the topics already covered by them, also adding topics that have not been discussed. More in detail, in the present 
survey the connectivity issues are also related to proposals for resource allocation and optimization, relaying and routing, cooperative networks and path planning and optimization strategies. A more thorough throughput analysis is carried out at network layer, more focusing on optimization strategies and covering also other aspects at physical and link layers. Channel modeling and characterization is described with reference to position (including the UAV height) optimization strategies, adding the discussion on frequency-related modes, and collecting and describing more papers that propose an analytical representation and characterization of the environment. Communication technologies are broadly discussed, including mmWave, CR, LTE, FSO and MTC systems. Finally, papers on modulation techniques, not present in all the other surveys, are here introduced and described in detail.

\subsection{Lessons Learnt}

Important lessons emerge from the analysis of the physical layer aspects covered in the IoD papers.

The modulation techniques analyzed for UAV-based networks are not novel by themselves. The main novelty lies in their application in the FANET context. As known in fact, drone detection and classification is a very important issue for UAVs, especially to face security issues, and this task can be accomplished by detecting the modulated signal at physical layer as testified by some of the surveyed papers. Efficient modulation techniques borrowed from other wireless network systems like LTE also help to increase the efficiency of the transmitted signal, with particular respect to noise reduction and transmission power optimization.

The analysis of the drones connectivity and channel modeling are tightly bound together, and are both important to increase coverage performance, to optimally allocate resources at different layers of the protocol stack, and to increase the path reliability in routing, path planning and position optimization strategies. Nevertheless, connectivity and channel models and schemes tend to be not so accurate because they cannot take into account jointly several aspects (the presence of obstacles in urban environments, signal dispersion and interferences, height and mobility of drones, etc.), thus being valid only under simplifying hypotheses. Furthermore, the optimization problems for resource allocation, path planning and routing algorithms bring to solutions that are computationally expensive, or suboptimal.

Analysis and optimization of throughput allows to increase the system performance in data exchange and to optimize different metrics in IoD systems (mutual distance, positions and paths of drones, spectrum and energy efficiency, network 
topologies). Also in this case, the throughput optimization in most cases translates into solving an optimization problem, whose solution is computationally expensive, or found approximately, or only in simplified scenarios. Furthermore, throughput maximization is counterbalanced by a higher energy consumption, which can become a serious drawback for battery-powered drones.

Performance of networks of drones can be increased also by choosing the most appropriate communication technology, depending on several factors like the specific environmental scenario, type and duration of tasks, communication range and energy consumption of drones, etc. Several works on this topic are mainly review papers; the most interesting aspects raised by papers on this topic lie in the exploitation of peculiarities of the chosen technology to optimize specific transmission aspects like spectrum utilization, data rate, and distance of nodes.

\section{Data Link Layer}

In this Section, some relevant issues at link layer are discussed. They are mainly focused on two different aspects: (i) the allocation and optimization of resources, and (ii) data scheduling. The Section closes up highlighting the lessons learnt on the theme. The overall organization of this section is reported in Figure 9.

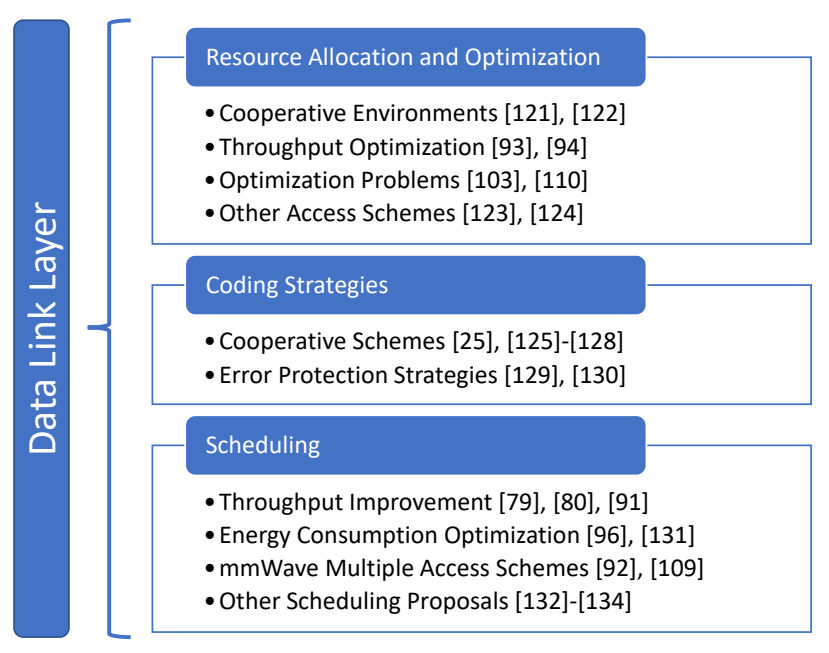

Figure 9: Data Link Layer Taxonomy. 


\subsection{Resource Allocation and Optimization}

Papers on resource allocation at link layer are mostly focused on the optimization of different performance metrics, such as PDR, packet error probability, bandwidth, delay, throughput, and energy efficiency.

\subsubsection{Cooperative Environments}

Schemes at Medium Access Control (MAC) layer in cooperative environments are proposed in [121, 122]. A packet loss tolerant algorithm is proposed in [121] to mitigate the communication disruption and help improving cooperation in networks of robots. The proposed algorithm aims at accomplishing a specific task despite link losses, guaranteeing cooperation and coordination among wireless networked robots. The network of robots is composed by one leader and several followers, and the goal of the algorithm is to set position, velocity and control of the followers so that they are always able to follow the leader. Depending on the amount of packet loss, the control input is periodically corrected to reduce the error, or bias, that each robot accumulates in the link with the other robots. A MAC protocol is proposed in [122] to reduce the packet error probability in WSNs where a UAV collects data from sensor nodes. The proposed protocol is part of a data collection scheme that exploits cooperation between a UAV and different sensor nodes. In this protocol, sensor nodes are allowed to transmit data based on a polling signal that gives them the chance to transmit data, which can belong to the single sensor or come from cooperative sensor nodes that collect data from other nodes. The MAC protocol manages also the construction of the cooperative sensor node list and establishes which data must be retransmitted through the chosen cooperative sensor node.

\subsubsection{Throughput Optimization}

Schemes for throughput optimization are presented in [93, 94]. A data acquisition framework is proposed in [93] to increase the efficiency of data gathering in UAV networks. The main goal is the optimization of the system throughput through a scheme that assigns different priorities to UAVs in different areas. To this end, a modification of the contention window mechanism at MAC layer is proposed, that lowers the range of the contention window sizes to areas with high priority, and increases it for lower priority areas. This approach allows reducing packet collisions and losses, especially when the UAV moves in the forward direction. A resource allocation method is presented in [94] for a wireless network composed by UAVs and GSs. Exploiting the Time Division Multiple Access (TDMA) scheme, resources are allocated in the communication channel between 
the UAV and the GS by taking into account mission-specific and environmental requirements. The beforehand knowledge of the UAVs throughput, that depends on the UAV specific mission, is exploited in the TDMA-based allocation scheme, to derive the optimal allocation of the different time slots and respect the throughput requirements of each UAV. Two different priority degrees of the UAVs are also considered in the time slots assignment.

\subsubsection{Optimization Problems}

The mechanisms proposed in the works [110, 103] bring to constrained optimization problems that can be solved through standard methods and/or approximations with reduced computational complexity. Several Multiple Access (MA) schemes are analyzed in [110] for mmWave UAV cellular-connected networks, with respect to energy efficiency. Two specific MA schemes, i.e., Rate-Splitting Multiple Access (RSMA) and Non-Orthogonal Multiple Access (NOMA), are analyzed in detail and optimized to maximize the energy efficiency for mmWave DL transmissions, taking into account real propagation patterns from antenna elements in last-generation wireless networks. The energy efficiency optimization problems for both the MA schemes are computationally very expensive, so an approximate algorithm is proposed to provide a solution. The work [103] proposes a resource allocation mechanism aiming to minimize mean packet transmission delay in a cellular network with UAVs placed in a 3D multi-layered structure. Delay is first derived analytically through a queuing system analysis that models the mean packet arrival rate. Then, it is minimized by solving an optimization problem that allocates spectrum and power for each layer in a two-layer UAV network, through standard procedures.

\subsubsection{Other Access Schemes}

Other access schemes for resource optimization are developed in [123, 124]. The goal of the access scheme proposed in [123] is the optimization of the accuracy in the medium access procedure. The proposed scheme helps an UAV joining an ad-hoc UAV network by correctly identifying the MAC protocol without demodulating the property field MAC header, which can be very difficult in particular situations (i.e., when channel attributes strongly change due to interferences). The identification of the MAC protocol is performed adaptively through machine learning methods that include a classifier able to identify MAC protocols according to features extracted from the channel attributes, improving the access accuracy. 
The paper [124] proposes a MAC layer protocol that optimizes the data transmission by sharing the information on the reciprocal position among UAVs. This information is useful to reduce the packet overhead at MAC layer, and to exchange control packets between neighboring nodes with the goal of increasing the effectiveness of mutual communication between drones and pointing directional antenna of transmitting UAV towards the UAV receiving data, so that the link can be established quickly, the delay in data reception is lowered and the throughput increases.

\subsection{Coding Strategies}

Some papers analyze and propose coding strategies at link layer for UAVbased networks [125, 126, 25, 127, 128, 129, 130]. Most of the surveyed papers on this topic analyze Network Coding (NC) [125, 126, 25, 127, 128]. NC is a technique that aims at saving time and bandwidth resources when trying to recover information from packets transmitted at link layer. It relies upon the principle that a transmitted packet includes kind of "repair information" for previously sent packets. The destination node uses this received repair packet in combination with previously received packets already stored in the receiving buffer, to recover the original information contained in the previously received packets. This concept can also be extended to multi-hop environments, since the information in a repair packet can be properly encoded so that it can be exploited by multiple nodes. The advantage of $\mathrm{NC}$ techniques is that the transmission time of a repair packet is usually much smaller than the time needed for an independent transmission of several packets, thus saving time and bandwidth, and increasing spectral efficiency [25].

\subsubsection{Cooperative Schemes}

In the context of UAV-assisted networks, $\mathrm{NC}$ is usually exploited in combination with cooperative techniques in multi-hop scenarios [25, 125, 126, 127, 128]. Cooperation is a topic peculiar of the network layer (as pointed out in Section 6.1); nevertheless, the NC techniques analyzed in these contributions are developed at link layer. Network coded scenarios are tackled in [25]. This work proposes a strategy to improve the QoS in UAV-aided networks that transmit high-quality video streams. QoS is evaluated in terms of reduced delay and increased throughput. in this context, NC acts in combination with an application layer ARQ protocol. In this paper, $\mathrm{NC}$ is applied at link layer, and relies on a cooperative technique where several nodes adopt a broadcast transmission of packets. Each node stores multiple packets, and performs an XOR operation among them to build a single 
coded packet, and forwards it to a destination node which, in turn, decodes the packer XORing it with the packets already stored, thus increasing the spectral efficiency. This coding technique is used to code packets coming from the same flow in a video stream, and is implemented in combination with a selective-repeat ARQ protocol at application layer, to increase the overall network QoS in dynamic UAV-based topologies. The works [125, 126] focus on a drone acting as a relay node in Network Coded Cooperation (NCC) for wireless networks. The adoption of an UAV as relay node gives a higher degree of freedom in terms of mobility. As a relay node, the UAV receives packets from different sources, in different time slots, performs NC over all the received signals, and transmits the coded packet to all the destinations. Based on the statistical distribution of the fading channels linking the sources, the UAV and the destinations, the contributions of these paper is to treat this problem analytically, by deriving expressions for the network coded noise and its variance, the overall SNR at destination node and the rate [125], as well as a closed-form expression for the outage probability and the impact of drone height on the outage probability [126]. Cooperative multi-hop networks of drones are analyzed in [127, 128] for data exchange by means of NC. An Instantly Decodable Network Coding (IDNC) technique is proposed in these works, whose goal is to reduce the energy consumption and delay due to the $\mathrm{NC}$ operations in a cooperative multi-hop network. This problem is modeled through a cooperative game theoretical approach [127, 128]. The NC metrics, such as decoding delay, completion time, delivery time and rate, are considered in the optimization problem and contribute to partition the network into coalition groups that take into account energy efficiency, together with delay. The main contribution in [127] is to define a scalable, low-complexity approach for the cooperation in the IDNC game that takes into account energy consumption. This approach is further enhanced in [128] by decentralizing the dynamic choice of the transmission power by each of the network nodes; furthermore, the stability of the cooperative game is studied, demonstrating its convergence to a stable coalition structure.

\subsubsection{Error Protection Strategies}

Other coding strategies are useful to protect information from errors and noise, as testified in papers [129, 130].

A packet coding strategy is adopted in [129] for delay constrained applications transmitting video over fading channels. The proposed scheme makes use of the so-called cross-packet coding strategy, that is based on several transmissions of the same information message occurring from the moment of message availability to the message decoding deadline. During this period, the subsequent 
retransmissions of the message are coded with different code rates, so that additional protection bits in subsequent retransmissions can contribute to increase the probability of successful decoding of the message. Differently from the conventional cross-packet coding strategies, the proposed scheme does not consider any feedback and does not remove protection bits relative to the previous transmissions, so to reduce the retransmission delay and keep a low frame error rate.

A convolutional denoising encoder is exploited in [130] to reduce noise due to the drone flight and the wind when transmitting recorded audio signals in UAV sensor networks. It makes use of convolutional neural networks that are trained with datasets obtained by mixing drone flying and wind noises with speech signal. The time-frequency features of the speech signal are extracted and used to eliminate the noise contribution from the target speech.

\subsection{Scheduling}

Scheduling of information exchanged among drones is another challenge usually tackled at link layer. Papers on this topic schedule the data transmission so that specific metrics, i.e., successful data rate and throughput, energy consumption and interference among drones, can be optimized.

\subsubsection{Throughput Improvement}

Scheduling at link layer can be useful to optimize communication throughput, as testified by [91, 79, 80]. A radio access scheme thought for improving relaying capabilities in a multi-hop UAV-based network is proposed in [91]. In this scheme, the number of DL and UL subframes scheme is dynamically changed by first changing the structure of the frame, and then scheduling the UL and/or DL subframes allocation in the frame to each node, so that the throughput of the communication links is improved also for relatively long distances between the communicating nodes. A UAV-aided network is studied in [79, 80] where a UAV is used as a flying BS to serve a group of ground users. A scheme is proposed in [79] that maximizes the minimum average throughput among all users by jointly optimizing the scheduling of data sent by users and the UAV trajectory, given a periodic/cyclical TDMA scheme of fixed period. The optimization problem is solved through an iterative algorithm that alternatively optimizes the user scheduling and UAV trajectory at each iteration, and whose convergence to the optimal solution is proven to be guaranteed. The same network scenario is presented also in [80], but with the addition of a ground BS to the UAV and the ground users. The performance metrics optimized in [80] are the same of [79] (average throughput 
and UAV trajectory), with, in addition, the users partitioning between the UAV and the ground BS.

\subsubsection{Energy Consumption Optimization}

Also the energy consumption can be reduced through effective scheduling, as illustrated in [96, 131]. Another scheduling algorithm is proposed in [96] to optimize the energy consumption in cooperative networks of drones with relaying capabilities, at the same time guaranteeing a target rate of successfully received packets. The goal of the algorithm is to schedule the transmitted packets so that the energy consumption is minimized, at the same time guaranteeing a target bit error rate. The problem is solved by means of integer programming techniques, even if its solution is NP-hard. A suboptimal algorithm is then proposed to reduce the computational overhead due to the problem complexity. A packet scheduling strategy is proposed in [131] for UAV-aided WSNs, where multiple UAVs relay data from a WSN sensor to a ground BS. The proposed scheme aims at saving energy in presence of lossy channels, and at increasing the UAVs lifetime. The scheduling mechanism relies on the channel quality indications reported by the UAVs to the BS, which in turn establishes the optimal subset of packets that each UAV must send to the BS, so that a target packet success rate is guaranteed, also minimizing the maximum energy consumption of all the UAVs. Since the optimization problem is NP-Hard and very expensive computationally, a suboptimal solution is proposed that achieves a faster convergence.

\subsection{3. mmWave Multiple Access Schemes}

The works [109, 92] study users scheduling in multiple access schemes for UAV communications adopting the mmWave technology. A study on SpatialDivision Multiple Access (SDMA), or Beam-Division Multiple Access (BDMA), in mmWave communications is carried out in [109]. The scheme is based on a BS equipped with multiple transceivers, and each UE equipped with a single transceiver, so that multiple users can transmit over a single beam and the achievable rate can be boosted. The main challenges of such a scheme are described, i.e., how to wisely schedule different users into a group, and allow different groups to access simultaneously the BS while minimizing the reciprocal interference. Multiple access for mmWave communication in UAV-aided $5 \mathrm{G}$ networks is analyzed in [92]. The proposed scheme takes into account the transmission of multiple highly directional beams peculiar of mmWave communication, allowing access

to different users for each beam, both for the UL and DL links. The goal of the scheme is to adapt the beamwidth to the channel quality indication and transmis- 
sion requirements of users, to establish the best user grouping strategy that selects the users that have to concurrently transmit data in a single beam. The algorithm proposed aims at improving the overall system throughput.

\subsubsection{Other Scheduling Proposals}

Other scheduling proposals at link layer are found in [132, 133, 134]. [132] proposes a scheduling scheme that takes into account bandwidth requirements during both upload and download phases as a function of services requests to provide dedicated tradeoffs. The simulated solution is a priority-based service scheduling scheme that able to increase service quality while granting fresh data service and lowering the average request serving latency.

The paper [133] proposes different data collection algorithms in a wireless sensor network assisted by an UAV. The goal of the algorithms is to increase the efficiency of data collection by taking into account transmission data rate and contact duration time between the sensors and the UAV. Based on synchronization and join messages between the sensors and the UAV, contact duration time, data rate and available time slots are derived. This information is then exploited to schedule the assignment of the time slots available to the single sensor to transmit data. The effectiveness of the algorithms is evaluated with respect to number of collected packets and fairness in data transmission among sensors.

In [134] a fault detection and scheduling mechanism is studied for a drone that exchanges information from different sensors through a communication network. The focus of this study is the transmission of information on fault detection in drone motors. To this end, the scheduling mechanism proposed in this work aims at preserving as possible the medium access, also enhancing the accuracy and rapidity of information exchange among the different parts of the system. Communication constraints and the possibility of packet drops are also taken into account.

\subsection{Comparison with Other Surveys}

The surveys discussing link layer aspects for drones communications are found in [8, 3, 13]. In [8] the surveyed papers on data link layer are analyzed with the

only focus on energy consumption aspects. The classification of the related approaches is based on the way energy is saved, which nevertheless is not the only issue of UAV communication at link layer. Link layer approaches are mentioned in [3] for the formation of UAV ad-hoc networks. In this work, the main challenges, i.e., synchronization among nodes, delays, resource reservation, access management and error control, and modified MAC protocols are summarized, citing only 
one paper that overviews them. So, unfortunately, no any detailed discussion is carried out in [3] on this topic. In [13] some link layer issues are addressed with respect to the cooperative robotic networks. They are mainly related to the severe limitations of signal propagation, that require the design of new MAC protocols. Some works discussing the most promising approaches are discussed, ranging from the well known TDMA, Frequency Division Multiple Access (FDMA) or Carrier Sense Multiple Access (CSMA) schemes, together with Medium Access Collision Avoidance (MACA) and hybrid schemes, with an additional analysis on the amount of control information for channel reservation. The main drawback of this classification is that it refers to the only cooperative robotic networks operating in underwater environment, and the surveyed works mentioned in [13] do not basically propose any novel technique at MAC layer.

If compared to the papers mentioned above, the main goal of this section has been to integrate and further develop their analysis, presenting all the most recent approaches discussed for UAV networks in a broader range of link layer related topics.

\subsection{Lessons Learnt}

The analysis of the proposals discussed in this section allows to acquire important knowledge on the pros and cons of resource optimization and scheduling strategies at link layer, as well as coding strategies. Algorithms proposed to reduce packet losses and increase throughput are important to improve the performance of communication links especially in critical scenarios. Nevertheless, significant modifications to MAC layer protocols are needed (i.e., the variation of some protocols parameters, additional prediction/estimation algorithms or metrics, etc.), that can create interoperability problems with the UAV-based networks that adopt the classical link layer protocols. Also cooperative schemes are useful to improve communication efficiency and reduce packet losses at MAC layer, but they introduce a data overhead needed to share information in the cooperative nodes. This complicates the implementation of scheduling mechanisms needed to decide which node must transmit the information, when, and at what frequency. Tight synchronization mechanisms are another critical point that raises in this scenario. Another consideration is that the optimality of resource allocation and scheduling often translates into finding the solution of complex optimization problems, whose convergence si not guaranteed, and that can be solved only at the cost of simplifications, that nevertheless bring to suboptimal solutions. Coding strategies are helpful to increase the efficiency of the communication link, in terms of time 
and bandwidth saving, and increased spectral efficiency. They are always associated to cooperation strategies, to increase QoS metrics in cooperative networks. The drawback of this strategy is the additional time needed to decode packets, and the increased computational complexity for encoding and decoding operations, if compared to the simple packet retransmission. This explains why the research efforts are actually focused on low-complexity techniques, that can scale well in networks with a large number of UAVs.

\section{Network Layer}

The present Section focuses on: (i) cooperative strategies, (ii) routing protocols, and (iii) relaying schemes. The organization of this section is reported in Figure 10. In particular, this Section aims at discussing all the main aspects and

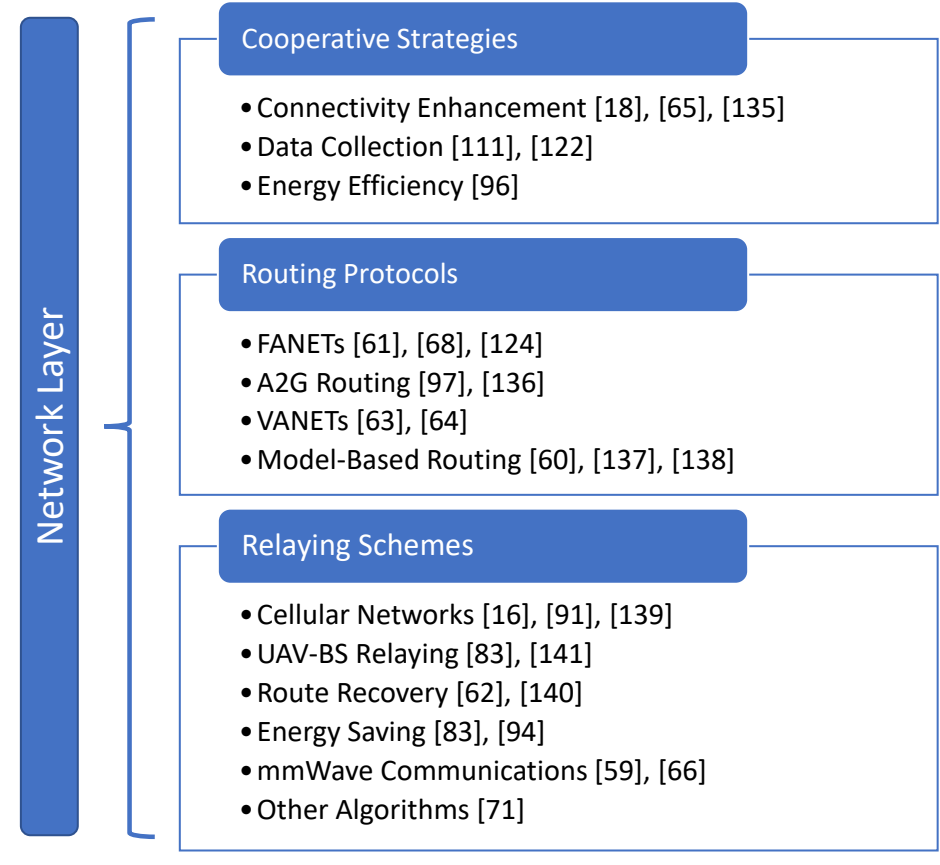

Figure 10: Network Layer Taxonomy.

issues related to the network layer of the IoD i.e., all the proposals and strategies in the multi-hop flying network scenarios. 


\subsection{Cooperative Strategies}

Cooperative strategies are discussed in [135, 65, 18, 122, 111, 96] to optimize different metrics, i.e., connectivity [135, 65], energy efficiency [65], data collection [122], coverage performance [111], and throughput [18].

\subsubsection{Connectivity Enhancement}

An architecture for ICNs that uses moving routers is proposed in [135]. An UAV that acts as a Content-Centric Network (CCN) router is used to deliver contents between physically disjoint networks. The goal of this proposal is to exploit the flexible capabilities of $\mathrm{CCN}$ routing, i.e., the utilization of content caches and content names, to improve communication performance at network layer, at the same time providing connectivity to disjoint networks thanks to the capability of the router to move autonomously. The scheme is also extended by proposing a communication architecture which uses different UAV-based routers to cooperatively process routing among disjoint networks.

A low latency routing algorithm is presented in [65], for a UAV-based cooperative network providing IoT services. The proposed algorithm is suitable for large scale networks of UAVs, where the latency of the classical routing algorithms developed in literature increases, and the connectivity decreases because of high interference and delay, together with the rapid topology changes due to the high mobility of UAVs. The Low Delay Routing Algorithm (LDRA) proposed in this paper exploits the cooperation among UAVs that have relaying capabilities,. It uses only partial information on UAVs location and connectivity, distributing the different data flows by optimally choosing the relay UAVs, so that latency is minimized.

A contention-free geo-routing scheme is proposed in [18] to decrease latency and increase throughput in UAV systems. The scheme is based on a cooperative relaying strategy, where multiple nodes concurrently transmit the same frame towards the destinations. In the first hop of the routing scheme, a source transmits a frame into the network, and nodes closer to the destination relay concurrently the frame. Thanks to the OFDM transmission, the multiple copies of the same frame more easily bring to a successful frame decoding. In the second hop, each receiver decides whether to forward the frame or not, based on both the position information provided by transmitters of the previous hop and the positive progress towards the sink. Due to the concurrent transmission of multiple nodes, the communication range and throughput increase, and latency decreases. 


\subsubsection{Data Collection}

A cooperative scheme is studied in [122] for efficient data collection in WSNs that use UAVs as relay nodes. Even if the main focus of this work is the proposal of a MAC protocol as described in Section 5.1, a cooperative architecture is also proposed for the data collection system. The scheme is based on a model where an UAV collects data from sensors along its flight path and provides them to cloud servers. The servers have complete knowledge of sensor nodes positions and data, and generate the flight paths of UAVs, sharing them with the UAVs. In the proposed scheme, the UAV requests the retransmission of missing data from cooperative sensors, which are collected in a list.

The cooperative scheme proposed in [111] aims at increasing the coverage performance of UAV-aided cellular networks where the UAVs act as BSs caching the most popular contents and providing them to mobile terminals, so that the ground BSs are offloaded. UAVs are grouped into cooperative clusters, to provide cached contents to a group of mobile terminals. Transmission of the cached content by the UAV in the cluster is possible only if some requirements, i.e., the amount of energy to feed communication modules, the presence of the requested content in the UAV cache and the maximum connection capacity of the UAV, are respected.

\subsubsection{Energy Efficiency}

Energy efficiency is the main focus of the cooperative relaying scheme proposed in [96]. The proposal is based on the adoption of a swarm of UAVs that relay data coming from remote sensors to a ground BS, to overcome the presence of lossy channels, save energy and extend the UAVs lifetime. The scheme is based on a relaying cooperative protocol that exploits the information on the reception quality reported by the UAVs to the BS. Accordingly, the BS schedules the packet load to be transmitted and the Adaptive Modulation and Coding (AMC) scheme for each UAV, to find the best trade-off between the success rate of packet transmission and the energy consumption. The optimization problem is NP-hard and mathematically intractable; thus an approximated solution is proposed that decouples the processes of energy optimization and AMC selection.

\subsection{Routing Protocols}

There are many works that propose routing strategies in UAV-based networks [124, 61, 68, 97, 136, 63, 64, 137, 60, 138], for different application scenarios and network architectures. 


\subsubsection{FANETS}

Papers [124, 61, 68] develop routing strategies for FANETs. Different communication protocols are proposed in [124] in the FANET scenario. Among them, a routing protocol is presented, which exploits a reinforcement learning approach. Specifically, it takes into account the UAVs positions in the routing policies, updating them by means of a reward function that depends on the network utility, so that the optimal paths can be chosen that minimize the delay in data delivery. The main advantages of the protocol are that a global knowledge of the network is not needed, and that the protocol has capabilities to continually evolve for selfoptimization purposes.

A predictive routing protocol is proposed in [61] to tackle the main challenges in FANETs, i.e., UAV speed, connection loss, changing network topology, etc. The protocol is based on a predictive $3 \mathrm{D}$ estimation of the expected connection time between two adjacent intermediate nodes with directional transmission, with a continuous update of position and speed information to improve prediction accuracy. When the estimated connection time is close to expire, an alternative path is chosen during data transmission, to guarantee service continuity. The adoption of omnidirectional transmission and beam-forming is also allowed; the former to increase the connection among nodes, and the latter to increase the transmission distance and reduce the packet collisions and the set-up time of the routing paths.

A modified routing mechanism to evaluate the impact of drone replacement in FANETs is proposed in [68]. The main goal of this work is to study how to replace dynamically drones in a FANET without degrading the overall network performance, and taking into account the network main characteristics, i.e., its dynamic variation in time, its support for heterogeneous traffic, and its limited lifetime. In the last part of this paper, after a description of the most used routing protocols in FANETs and their classification into reactive and proactive protocols, a modification of the proactive routing protocol chosen for the analysis is proposed, that adapts a protocol attribute to the battery charge of the node, so that the connection during handover between UAVs (because of battery depletion) can be maintained.

\subsubsection{A2G Routing}

In [97, 136] routing strategies are proposed in in Air-to-Ground (A2G) environments. The paper [97] proposes a centralized routing protocol for networks of UAVs controlled by a Ground Control System (GCS). In the proposed scheme, all the drones are managed by the GCS, which is the component demanded to collect and manage control-related information from drones, including their routing 
tables. Such an approach avoids the continuous exchange of periodic information on the link cost values among UAVs (the GCS can extract this information from the geographic information of UAVs). Furthermore, the disconnection time of the network due to the UAVs mobility can be minimized because the GCS knows the schedules of drones mobility and can predict the topology changes. The proposed protocol is implemented using real UAVs and GCS.

Two different opportunistic routing protocols are developed in [136] in a scenario where a UAV moving at a predefined height and speed provides connectivity to different mobile sensors, that move in the same direction with different speeds. Opportunistic routing is suitable for scenarios with a changing network topology, because of its capability to increase throughput. In both the proposed protocols nodes that want to transmit data but are not in the UAV communication range, choose the neighbor nodes in the UAV range that can act as packet forwarders, with the goal to increase the PDR. In the first proposed protocol, a source node transmits packets to all its neighbors in its range, while in the second it transmits packets to the forwarder with the highest velocity. The PDR increase comes at a cost of an increased delay and routing overhead, if compared to the direct connection between source nodes and the UAV.

\subsubsection{VANETs}

Routing protocols for VANETs are developed in [63, 64]. For sake of better clarity, Fig. 11 provides an illustrative example of a UAV-assisted VANET where routing operations and protocols are applied. In a nutshell, drones are herein assumed to monitor a road as part of an ITS. Once a situation of interest is detected, i.e., an accident, the ground control infrastructure is in charge of providing updated information to the all the vehicles on the road. Thanks to the employment of Variable Message Sign (VMS) systems, and leveraging Vehicleto-Infrastructure (V2I), all the road users can be promptly notified about the dangerous situation and, at the same time, the closest available UAV can be relocated, when needed. As will be discussed later on in this section, ITSs are also using Vehicle-to-Vehicle (V2V) communications. In fact, connected cars and direct links between the drones and the vehicles may be used to simplify message exchanges, thus lowering latencies and improving communication effectiveness.

The routing protocol developed in [63] aims at increase the reliability of data delivery for UAV-assisted VANETs in urban environments. The proposed protocol is based on the information exchanged between UAVs and vehicles on the traffic density and the connectivity of the vehicles. Based on this, the UAVs can decide their location to relay data in the points of the network where connectiv- 


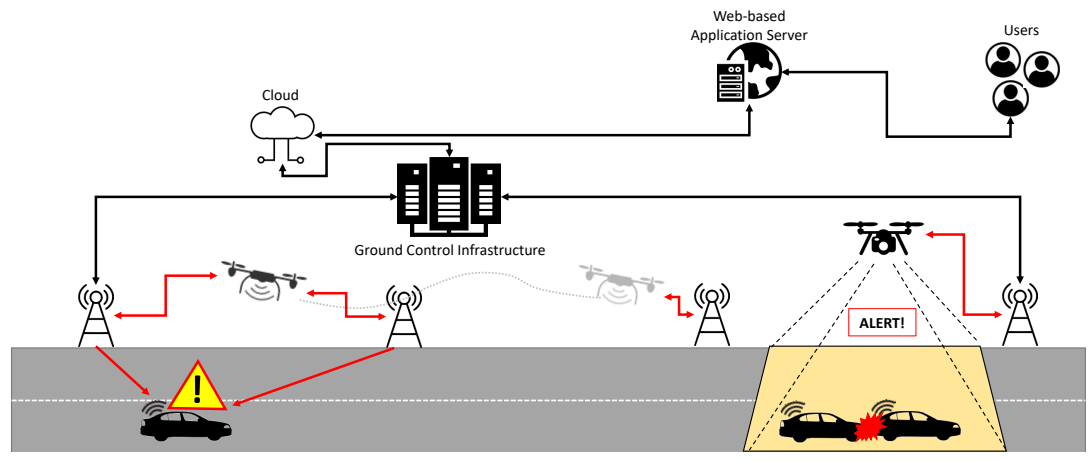

Figure 11: Drones in a ITSs scenario with connected vehicles.

ity among ground vehicles is missing because of the presence of obstacles which are present in typical urban environments. Accordingly, vehicles can choose to use UAVs as forwarders if there are no available paths in direct vehicle-to-vehicle communications.

A routing protocol that finds the shortest end-to-end connected path in VANET environments is proposed in [64]. It takes into account the high mobility of vehicles and the frequent changes in the network topology due to unpredictable movements of vehicles. The proposed protocol exploits heterogeneous communications through the cooperative interaction between an ad-hoc network of UAV and a VANET. The goal of the UAV network is to restore communication links that fall down because of the presence of obstacles in the urban environment. To this end, two different protocols are used: the first, more delay-tolerant, exploits the global knowledge of the UAVs about the connectivity status of the road segments to route data between UAVs and ground vehicles; the second, more reactive, routes data among UAVs only when needed.

\subsubsection{Model-Based Routing}

The routing protocols proposed in [137, 60, 138] are based on analytical models. A routing protocol for networks of UAVs is proposed in [137]. It exploits an application layer functionality that predicts future trajectories of UAVs and that is integrated in the a routing protocol already known by literature, to improve connectivity in the UAV-based network and reduce packet losses. Different mobility models and exploration algorithms are used for comparison between the proposed protocol and other known routing protocols.

A routing framework for hybrid space/air networks is proposed in [60], to mitigate the high bit error rates and long delays that can occur because of the mobility 
of nodes and the varying network topology. The framework exploits a routing algorithm based on a hybrid time-space graph, that is described by two subgraphs. The first subgraph describes deterministically the space network. The second subgraph aims at predicting the contact time and contact probability among the UAVs of the air network, and for this reason it is based on a semi-Markov prediction model. The graph is transformed into a state-space graph to establish the optimal next hop, based on a forwarding rule that can exploit UAVs in the air network or satellites in the space network as relays. The goal of the proposal is to improve the message delivery ratio, the end-to-end-delay and the power consumption.

The routing algorithm proposed in [138] exploits 3D cubes as partitioning regions to forward data among UAVs. More specifically, data are relayed by choosing only one UAV as relay node per region. The optimal path among different cubes is chosen based on the maximum successful transmission probability derived from an analytical model. Then, the most appropriate UAV chosen to relay data in each cube is selected by jointly considering again the successful transmission probability and the UAVs mobility. The goal of this routing algorithm is to improve the end-to-end delay, jitter, and PDR.

\subsection{Relaying Schemes}

UAV-based relaying schemes are presented in [16, 139, 91, 140, 62].

\subsubsection{Cellular Networks}

The papers [16, 139, 91] exploit drones as relays in cellular networks. The review paper [16] analyzes D2D communications in different application scenarios. Part of this work is focused on the utilization of drones as D2D relays, to extend cellular network coverage especially in extraordinary conditions when the ground BSs can be damaged or switched off in critical scenarios (like catastrophes, network damage, etc.). In such situations, drones transfer the signal from far BSs to mobile devices, filling coverage holes. This paper does not go into deeper details on the implementation of D2D relaying functionalities. The same topic is described also in [139], that tackles the wireless coverage issue in case of disasters or incidents in public safety wireless networks. This work proposes a multi-hop D2D scheme that extends network coverage wherever ground relay is not possible. The proposed scheme jointly finds the optimal UAV position and the resource allocation, in time or frequency domain, so that the data rate of each hop in the path from the BS to the mobile device is maximized.

A radio access scheme is proposed in [91] for muti-hop relay networks composed by UAVs. This scheme increases the efficiency of relay communications 
between an observation UAV transferring data to a ground BS exploiting UAVs as relay nodes. This study is based on the assumption that the time-division relaying technique exploited in LTE-A can be suitable to increase the efficiency of relay communications among UAVs, in terms of both reduction of mutual interference among UAVs and communication order by the relay node to transfer data from the observation UAV to the BS, especially for long distances. Since the existing LTEA scheme is not suitable for UAV-based relaying because of some limitations in the frame structure, this work proposes an LTE-A access scheme that increases the relay communication efficiency over long distances, taking also into account the UAVs height.

Also the solution presented in [139] exploits a relay UAV, called Floating Relay (FR), to dynamically and adaptively provide additional coverage in a macrocell served by a ground BS to tackle the issue of an increasing traffic volume in the macrocell. The discussion focuses on some important aspects like frequency reuse, the interference both between FR cells and the macrocell and among FR cells, bandwidth allocation for the backhaul network, and coverage capabilities.

\subsubsection{UAV-BS Relaying}

Other relay schemes exploiting UAVs for data transmission to a ground BS can be found in [141, 83]. The relaying scheme proposed in [141] is conceived for image transmission in an UAV-based network. In this work, some survey UAVs transmit sensed images to a BS through a relay UAV. The scheme is based on a planning model that computes the best set of relay points where the relay UAV can meet the survey UAVs, that minimize the average traveling distance of the relay UAV. Then, a reinforcement learning approach is used to find the optimal time to visit each point. This technique exploits a reward function based on the rate of image acquisition. The proposed solution avoids the need of any collaboration between survey UAVs and the relay UAV, and reacts to the varying network traffic to improve the quality in end-to-end delay and frame delivery ratio.

Similarly to [141], the paper [83] focuses on a relay scheme where a relay UAV forwards data collected from multiple observation UAVs to a ground BS. The observation UAVs cover a wide area. Depending on the main parameters that influence the network performance, i.e., signal attenuation and obstacles, a formula for the upper bound of the throughput in the network is derived, together with the optimal location of the relay UAV that maximizes the upper bound of the throughput. 


\subsubsection{Route Recovery}

Relaying is adopted for route recovery schemes in [140, 62]. A relay scheme is proposed in [140] that aims to solve the problem of jamming attacks in VANETs. The scheme exploits an UAV that relays data from vehicles to RoadSide Units (RSUs) placed at fixed locations. If the RSU serving the vehicle is in a heavily jammed area, the UAV becomes in charge of relaying data to another RSU with better channel conditions. The UAV decides whether or not to relay data from the vehicle to another RSU which is far from the jammer, depending on the channel quality in the direct link between the vehicle and the serving RSU. The interaction between the UAV and the jammer is studied through an anti-jamming relay game approach, derived from the game theory. Reinforcement learning techniques are also used to derive the optimal relaying strategy of the UAV without knowing the jamming model.

A route recovery scheme is proposed in [62] in ad-hoc networks where UAVs act as relays. The scheme is based on probe packets sent by the UAVs to discover the route topology and stitch partial paths and avoid network holes in damaged networks. Based on the captured topology, an algorithm for optimal UAVs deployment is adopted, which minimizes the UAVs traveling time and distance and avoids duplicate coverage. It is exploited to decide how to replace he network holes through UAVs. To this end, an algorithm is adopted that dispatches a reduced number of UAVs, allowing an improvement of routing performance both on local and global scales.

\subsubsection{Energy Saving}

The relay schemes proposed in [93, 84] are focused on energy saving. A relaying strategy is proposed in [93] as part of a routing algorithm aiming to save the energy consumption in a UAV-aided WSN for data acquisition. The routing algorithm is based on a priority-based scheme, that takes into account the UAV mobility. More specifically, sensors are classified into frames, each one with an assigned transmission priority, so that sensors in more urgent areas transmit their packets with a higher priority. Sensors of a frame are further grouped into clusters, choosing a Cluster Head $(\mathrm{CH})$ that transmits gathered data to the UAV. The proposed routing algorithm aims to deliver data from sensors to the $\mathrm{CH}$ by choosing the optimal relay node with the better channel quality and the shorter distance from the $\mathrm{CH}$, so that the energy consumption from source to destination can be saved.

The relaying scheme proposed in [84] makes use of a single UAV that helps two ground stations without any direct connection to reach each other by follow- 
ing a circular trajectory. The goal of the proposed scheme is to maximize the spectrum and energy efficiency by jointly optimizing the time allocation of the UAV relaying, its speed, and its trajectory. The solution of this optimization problem reveals a trade-off between the energy consumption for the propulsion of the relay UAV and the maximization of energy and spectrum efficiency of the whole system.

\subsection{5. mmWave Communications}

The works [59, 66] propose routing schemes for mmWave communications. Mobile relays in mmWave communications are studied in [59]. A relay method suitable for mmWave communications is proposed, to bypass obstacles and/or increase the communication range. In this scenario, an UAV acting as relay adjusts dynamically its path based on real-time measurements of the link qualities of different mmWave beams. In this way, the UAV is able to dynamically choose its optimal position accurately and in a short time, despite the unpredictability and varying nature of the wireless link. The proposed scheme is also enhanced to multiple relays that can also use directional beams to extend the communication range.

A methodology for dynamic rerouting in networks exploiting mmWave technology is presented in [66]. The proposed methodology is applied to mmWave backhaul links that dynamically reroute depending on the channel conditions, to improve the flexibility and reliability of the backhaul solution. Dynamic routing is possible thanks to UAVs that work as aerial relay nodes, so that the negative effect of occlusions and signal blockage due to obstacles can be reduced. The methodology presented in this work considers the influence of some components, like the signal propagation model and the blockage probability of the mmWave links due to obstacles typical of urban environments, and the UAVs mobility.

\subsubsection{Other Algorithms}

The relaying algorithm proposed in [71] is applied in a wireless backhaul network composed by balloons. Some UAVs are used as relays to increase the network reliability, which fluctuates because of the limited control on the balloons mobility that can bring to temporary link failures. The proposed algorithm aims to plan the UAVs paths and schedule them all so that the network reliability is maximized. The path planning algorithm is developed for the single UAV and also for multiple UAVs, supposing to know the availability prediction of the links among balloons and the traffic matrix of the network. 


\subsection{Comparison with Other Surveys}

The network layer has been widely studied in the past literature, and related papers mainly discuss on cooperation, routing and relaying aspects at network layer of UAV-based networks. The survey papers that cover this topic are [3, 11, 8]. The work [3] analyzes different aspects of the network layer, ranging from cooperative networks, routing and relaying strategies. It carries out an exhaustive analysis of frameworks, models and approaches applied to multi-UAV cooperative systems, data routing and relaying strategies. Routing strategies are analyzed in [11] for networks of UAVs. In this work only data routing is analyzed in detail, presenting the most significant protocols in MANETs and VANETs. The survey [8] discusses the applicability of the most important types of routing protocols, whether static, proactive, reactive, hybrid or geographic, to the MANET scenarios. Networks prone to delays and disruptions are also analyzed, discussing the suitability of other kinds of routing protocols in this more challenging context. With this well-articulated background in mind, the main goal of the network layer analysis carried out in this section has been to update the discussion of network layer strategies in [3, 11, 8] through a detailed survey of the most recent literature on this topic.

\subsection{Lessons Learnt}

Multi-hop strategies at network layer have been proposed in many papers on networks of UAVs. The surveyed works discussing cooperation among drones testify that cooperation is a good solution to increase connectivity among separate networks, to increase the effectiveness of routing algorithms and the energy efficiency, and to reduce latencies in data exchange. Except for the papers proposing simplified scenarios, these improvements translate into optimization problems, whose optimal solution comes at a cost of high computational effort.

As expected, routing strategies are one of the main issues at network layer. This holds true also for networks of drones, where many challenges peculiar of FANETs (i.e., UAVs mobility, fast changes in networks topology, error-prone communication channels, etc.) must be tackled. To this end, the proposed approaches make use of strategies ranging from proactive and predictive techniques, to centralized architectures, analytical models, and information on UAVs position, velocity, etc. The key-aspect of such proposals is the optimal choice of the UAVs as data forwarders, so that the above-mentioned challenges can be effectively managed without degrading the network performance (in terms of PDR, delay, and routing overhead). Lessons learnt from the analyzed works demon- 
strate that the joint optimization of all these parameters is extremely difficult, and the most suitable routing strategy is strongly context-dependent.

Another important lesson is learnt from the analysis of the relaying schemes proposed. They are adopted for different purposes, i.e., extension of coverage and communication range, interferences reduction in $\mathrm{G} 2 \mathrm{G}$ multi-hop communications, energy efficiency, throughput optimization, and defense against jamming attacks. Studies in this direction show that relaying can be effective especially in environments subject to interferences from external sources (physical obstacles, jammers, etc.) or intrinsic to the specific short-range communication technology, as mmWave is. Most of the problems presented in the surveyed works are tackled by finding the optimal number and positions of relay nodes; but, once again, the optimization problems suffer from the same drawbacks highlighted for cooperation strategies.

\section{Transport and Application Layer}

The Transport and Application Layers have been conceived as independent. It is worth noting that, in most cases, transport layer is taken for granted. This is motivated by the fact that many applications are designed focusing on the expected performance level and, hence, QoS and QoE Indicators are used. Accordingly, no survey paper so far investigated application layer in detail. Indeed, several references start from the concepts of Network Function Virtualization (NFV), Software Defined Network (SDN) and/or distributed computing schemes to propose innovative schemes that demonstrate offloading possibilities in specific applications. At times, applications are conceived as solutions released on top of consolidated telecommunications technologies. In those cases, the protocol stack is not discussed in detail, nor it is modified. This happens frequently when LTE, 4G, or $5 \mathrm{G}$ communications or, more in general, consolidated networking solutions are involved. Overall, many scientific contributions rely on the Internet model, which involves Transmission Control Protocol (TCP) and User Datagram Protocol (UDP). Therefore the transport layer is not among the major concerns and is assumed to be TCP- or UDP-based. For all the scientific contributions that will be discussed in this section, a focus on the transport layer will be provided whenever the solution is different from the two cited above.

The present Section proposes application layer-related contributions, mainly connected to: (i) QoE, (ii) Computation offloading, (iii) Video Streaming, (iv) data collection and distribution, (v) events monitoring and management, and (vi) 
task allocation. The Section closes up highlighting the lessons learnt on the theme. In Figure 12, the overall organization of the present Section is summarized.

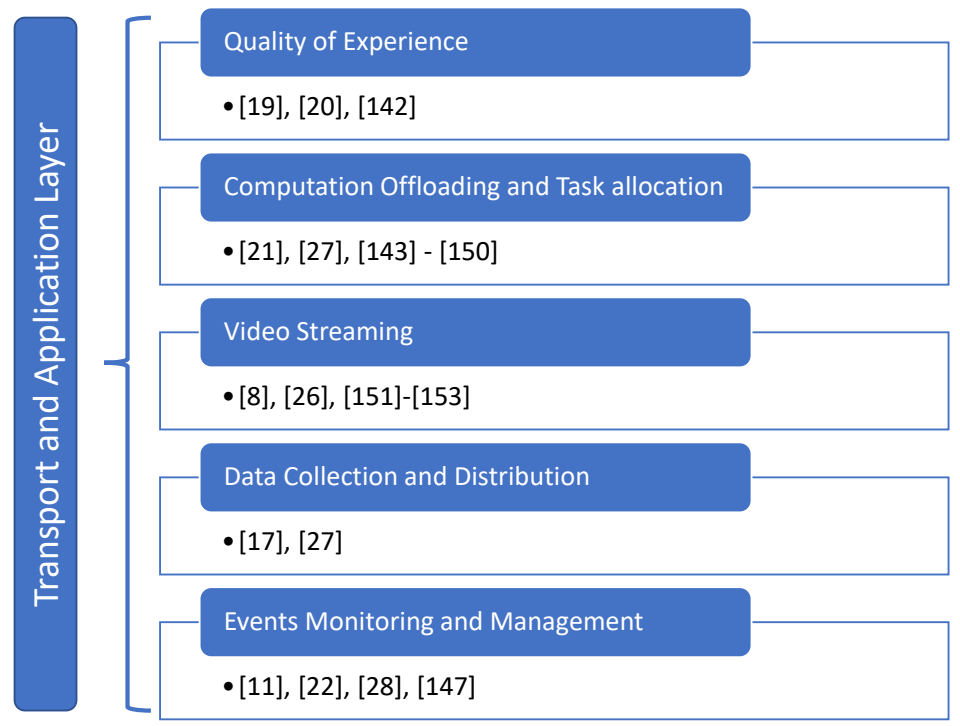

Figure 12: Transport and Application Layer Taxonomy.

\subsection{Quality of Experience}

In the IoD context, the network is specifically designed to transmit images and video recordings. For some applications, a very low latency may be highly required (i.e., real-time monitoring). In some other cases, instead, ultra high quality is needed. This heterogeneity leads to the definition of QoE indexes, which may enable several possibilities.

One of the most thrilling perspective in research for mobile telecommunication networks is represented by the possibility to employ UAVs as mobile BSs. In fact, with the possibility to mount a base station on top of an aerial platform, BS planning and deployment can be sensibly simplified. At the same time, this possibility may significantly improve methodologies for coverage area optimization. Further, mobile BSs could be helpful in solving several problems connected to base station capacity and inter-cell interference, which are among the major stakeholders when studying cellular networks. Those problems are tackled by a number of contribution proposed so far and, in particular [20] demonstrates the 
strengths and weaknesses of the employment of a deterministic approach to analyze these problems. In particular, this work analyzes the aforementioned parameters with a variable threshold for the received power. Accordingly, an optimal altitude and power consumption model is derived for aerial BSs in different environments which mainly refer to sub-urban, urban and highly populated urban environments. Among the main outcomes, a channel model for A2G communications is obtained.

Granting QoE does not specifically mean to improve a specific application and/or functionality. In fact, QoE analysis can also be used to design advanced coordination functionalities. In [19], QoE is considered in the context of heterogeneous networks, where mobile BSs are mounted on top of UAVs. In this work the flight plan is the output of a dedicated QoE-aware algorithm, based on the Qlearning approach. Simulation results obtained in this contribution testify that the flight plan diversification improves the overall QoE of the users. In the work [142] drones are configured in FANETs covering area where no fixed (i.e., ground) network infrastructures are deployed, or are hard to reach. The proposal tackles the lack of standardized routing protocols for FANET applications to allow efficient communication between devices thanks to an adaptive routing protocol based on fuzzy logic and demonstrates its added value in terms of both QoS and QoE.

\subsection{Computation Offloading and Task Allocation}

The tasks that drones can carry out may involve more than one drone at a time. This means that drones may be organized in swarms or clusters. Several contributions so far investigate the tasks that drones can handle suggesting that swarms may be a preferable choice when the application (or reference scenario) may benefit from the increased coverage capability a drone can offer. Even though a swarm of drones may represent an interesting opportunity, each of them is still limited in terms of computational power and energy resources. Such limitation suggests an optimization of the task list of the droned, for example by balancing the load of tasks among the swarm components. In this fashion, [143] proposes an opportunistic solution to provide computational offloading for a swarm. The scheme leverages the IEEE 802.11ac communication technology and is based on an artificial neural network with a prediction module specifically designed to provide updated decisions on whether it is preferable to keep the task list of a drone, or offload it. The method aims to be time-effective in deciding whether to offload tasks to other clusters or not.

The same problem is discussed in [144], where different swarms are supposed to have different task assignment lists. In particular, whenever one of the clusters 
is more heavily charged with respect to another, the proposed scheme opportunistically decides to carry out the offloading to another cluster, with respect to a number of different parameters and costs. The effectiveness of the scheme lies into an increased drone lifetime and a shorter response time.

The paper [21] deals with the problem of delay mitigation for video streaming applications, which is of great relevance int the context of cellular networks, especially in high traffic conditions. The proposal of this work is the design of algorithms and criteria for offload selection and drone positioning. This goal is reached by adopting UAVs that carry lightweight commercial micro-cells with small form factor, so to better handle congestion for macro-cells.

The problems of complex and time-consuming calculations to be carried out during a mission, together with the limited resources typically available on-board the UAVs, are discussed in [145, 146]. Here, computation offloading strategies are, once again, proposed as feasible solutions to mitigate the issues related to over-exploitation of constrained resources. The approach proposed in [145] is referred to as Mobile Edge Computing (MEC) and is based on the game theory, with a focus on a sequential game involving drones, BSs and edge servers acting as players with computation tasks. The contribution demonstrates the existence of a Nash Equilibrium and design an offloading algorithm to derive possible tradeoffs between energy consumption and achievable delay. In [146], the problems related to temporary overloading of the network are solved relying on Deep Reinforcement Learning (DRL) techniques.

In the context of surveillance systems, [147] discusses the theme of safetyrelated applications in hazardous locations. The proposal leverages the concept of computational offloading by proposing an Adaptive Computation Offloading Drone System (ACODS) solution, that is based on a response time prediction module for providing task offloading decisions. Task offloading management is achieved via a Multipath TCP (MPTCP) algorithm, a solution already known in literature, that is based on uses multiple communication interfaces to communicate and transfers the packets through one or multiple interfaces. The adoption of the MPTCP performance of the network is improved in terms of reliability, throughput, and delay.

Drones are employable in a number of industrial-grade applications, as argued in [27]. In this case, the discussion proposes an interesting point of view on the IoT scenario, by proposing an application that provides predictive maintenance to energy distribution systems through the employment of UAVs with visual capabilities combined with a $5 \mathrm{G}$ network infrastructure. In particular, thanks to the $5 \mathrm{G}$ communication network, massive capacity, zero delay, elasticity and optimal de- 
ployment can be reached in in scenarios with a massive deployment of IoT devices for broadband and mission critical services.

Drones employability has been discussed also in fog computing and MEC. In the former case, drones are conceived as enablers for distributed offloading intensive computation tasks to an edge/cloud server [148, 149]. For this reason, the paper [148] proposes a game theoretical approach in which drones are the players and the cost function to be minimized is a combination of energy overhead and delay. Thanks to this approach, drones can detect, identify and classify objects and/or situations dealing with intensive tasks such as pattern recognition and video preprocessing.

Due to the limited computing resources of drones swarm, it is usually difficult to handle computation-intensive tasks locally, hence the cloud based computation offloading can be adopted a sa solution. This become of greater relevance as long as low latency and high reliability are required. The proposal in [150] is based on fog computing for swarm of drones. Here, latency and reliability are taken as constraints of an optimization problem where the energy consumption is the optimization target function to be minimized.

\subsection{Video Streaming}

Drones employability in both civil and military applications include several possibilities such as search and rescue, coverage, and aerial imaging in different environmental scenarios, as illustrated in Figure 13. Once the drone is flying, gathering images and videos may not be as useful as it could be in video streaming contexts. This aspect may become more and more challenging when referring to a swarm of drones. In this case, coordination among the swarm is not simply beneficial, but becomes a need.

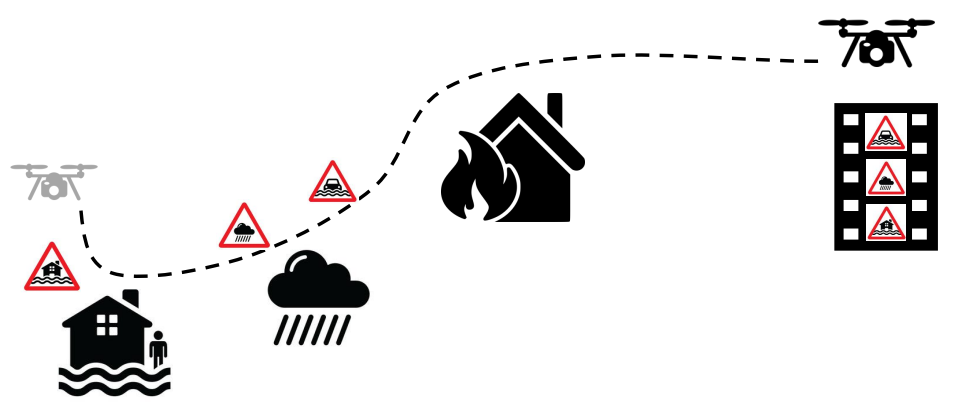

Figure 13: Drone gathering video streaming signals in environmental relevant scenarios. 
Several contributions so far describe the existing communication and routing protocols, including Ad-hoc On-demand Distance Vector (AODV), LocationAided Routing (LAR), and Greedy Perimeter Stateless Routing (GPSR) protocols [8]. These solutions have been deeply investigated in order to identify their limitations when applied to networks that include drones or swarm of drones.

According to the specific application scenario, the main requirements and characteristics may vary. At the same time, it might happen that drones are asked to fly over multiple areas of interest, which leads to the need for designing optimized trajectories to coordinate the flight of the swarm in a continuous way. Video streaming based on drones employability may be sensibly enhanced by leveraging multicast wireless video streaming to transmit data. In particular, video multicast streaming using IEEE 802.11 poses challenges of reliability, performance, and fairness under tight delay bounds [26]. The latter assumption in strengthened by the fact that "ready-to-fly" consumer drones use a fix-bitrate technique to encode video, for example, $1080 \mathrm{p}$ or $4 \mathrm{~K}$ resolution, which provides poor performance in high mobility conditions where the communication channel may be subject to sudden and relevant fluctuations. In this work, a dedicated section is dedicated to multi-homing. Since a multi-homed network is defined as a network is connected to multiple Internet Service Providers (ISPs), the proposed approach helps avoiding connection failures. To reach this goal, it is necessary to integrate in different layers of both the network and the transport layers in both Internet Protocol version 4 (IPv4) and Internet Protocol version 6 (IPv6) networks.

Video streaming may also be conceived not only as application enabler but as a method for handling and controlling network traffic and design. As a matter of fact, such a perspective enables interesting perspectives in terms of variable traffic demands, application heterogeneity, and application requirements. The contribution presented in [151] proposes dynamic cloud service placement to handle real-time video streaming and control commands for a drone that is controlled by a remote user. In this case, the main aim is to first grant, and then improve, the QoE for streaming services provided by drones using UDP. A similar problem is faced in [152], where the drone is remotely controlled and the high demands in both the UL and DL directions are thoroughly characterized in terms of QoS.

Another situation of relevance for video streaming and real-time surveying is data gathering from disaster-struck regions, as discussed in [153]. In fact, in all cases where the ground network infrastructures are damaged, drones may offer rapid deployment for data source recovery. Without loss of generality, the simple deployment may be ineffective without an adequate energy management strategy. The case study discussed in this work envisages a scenario in which drones operate 
alongside the wireless network infrastructure to establish a LoS link for communication while investigating a power allocation strategy for the BSs. As a result, the work highlights that it is of outermost importance to incorporate the multitier heterogeneous network to extend the network coverage in such challenging scenarios.

\subsection{Data Collection and Distribution}

Thanks to the high mobility of drones, their employability has been discussed in a number of applications, such as service delivery, pollution mitigation, farming, and rescue operations. On the theme, [27] proposes something similar to what discussed in [11]. In [27], in fact, UAVs are proposed as a value-adding utility to IoT devices and networks in the context of MTCs. The contribution represents an interesting survey of all the UAV-based architectures that can enhance smart sensors, cameras, actuators, and, more in general, IoT devices since they enable a brand-new perspective: an eye in the sky.

Data management is not only a matter of efficient upload (like happens in data gathering applications). In fact, [17] argues that a high rate, but also cost-efficient, easy-to-deploy, and scalable, backhaul/fronthaul framework, is of outmost importance in the context of $4 \mathrm{G} / \mathrm{LTE} / 5 \mathrm{G}$ communications and wireless networks. In this work mobile BSs are conceptualized as Unmanned Flying Platforms (UFPs). This definition is motivated by the fact that UAVs are only one of the kinds of flying platforms that can be used in this context. In particular, flying platforms may be drones or balloons. To provide advanced communications services in FSO, the proposal in [17] investigates the feasibility of a framework that involves both backhaul/fronthaul allowing traffic flows between the access and core networks via point-to-point links.

\subsection{Events Monitoring and Management}

In [11] the employment of UAVs is discussed to demonstrate their countless reference applications. In particular, public protection and disaster relief operations, but also for commercial and governmental services. Some good examples are surveillance and reconnaissance, public safety, homeland security, forest fire monitoring, environmental monitoring, security and border surveillance, farming, or even Internet delivery, architecture surveillance, goods transportation.

Fortunately, not all the scenarios and applications investigated in the scientific literature are related to dangerous or extreme situations. In particular, network capability could be ineffective/insufficient for temporary high loads. The contribution [28] deals with high-action sports game played on a large field. In such 
events, the idea of using networked drone cameras to gather real-time data can be considered as a great intuition, still challenging. In the design phase, it clearly emerges that distributed approaches yield sub-optimal solutions, an assumption that is motivated by the lack of coordination. Still, a centralized approach to coordination may imply round-trip latencies of several hundreds of milliseconds. For this reason, the contribution proposes a fog-networking based architecture that aims at coordinating a network of drones to capture and broadcast the sports game, with a tradeoff between coverage and streamed video bitrate. Here, latencies are mitigated by a centralized controller that leverages a predictive approach to upcoming locations in order to re-assign the UAVs to new locations, when needed. The work shows that relay nodes are able to boost throughput while working in real-time.

On the same theme, the work proposed in [147], and already discussed in this Section, can be mentioned once again since the proposal may contribute to highly advanced, and safe, surveillance of hazardous locations.

The paper [22] proposes a cloud-based system that is able to remotely control and manage all the operations carried out by drones and, more in general, robots. Since this work proposes an entire system with multiple components, the transport layer of each of them. As for the UAV, MAVLink uses UDP, TCP, Universal Serial Bus (USB) and serial. When UDP and TCP are used, data are forwarded to client applications through dedicated Websockets. The framework is able to operate in a wide range of commercial applications, under real-time constraints and below visual LoS conditions. Still, the proposal is facing security problems and future research directions involve both transmission and coordination tasks.

\subsection{Lessons Learnt}

This section is focused on all the technological aspects connected with application layer. However, several scientific contributions discussed have shown a peculiar influence, and non-negligible impact, on peculiar QoS parameters, such as latencies and PDR (or, complementary, Packet Loss Ratio (PLR)). These quantities have, in fact, a close link with the QoE parameters that can be used in quality assessments on streaming services, especially video. With respect to path planning, the impact on the application layer is relevant. In fact, optimizing the path that drones are programmed to follow during their mission has a significant impact on the optimization of onboard resources, with a consequent extension of mission longevity. Similar considerations can referred to the optimization problems connected to resource handling and data exchange capacity. 
The dedicated study of the problems connected with the application layer demonstrates that mobility is a key enabler for different applications in several scenarios. In particular, it is possible to guarantee local and temporary offloading thanks to the repositioning of the drones, both in the case of mobile BS deployment and when drones are part of a swarm. In the case of mobile BSs, they can be re-deployed even when a replacement is necessary. Thanks to these functionalities it is possible to think about adaptive, temporary and optimized coverage based on the needs of the application scenario. Some case studies have shown that the typical Key Performance Indices (KPIs) that are used to measure QoE and QoS can become input variables to objective functions and algorithms specifically dedicated to the design and optimization of routing. The latter represents an important frontier in the design of drone networks, especially when large swarms are involved. At the same time, a softwarization-based approach could be used to create customized solutions tailoring the specific application needs.

\section{Cross-Layer and Optimization Approaches}

This Section is specifically dedicated to the details on the following aspects: (i) Path optimization and collision avoidance, (ii) State estimation and optimization, (iii) Network formation and control, and (iv) Coverage analysis and optimization. All the aforementioned topics are peculiar to the application layer. Nevertheless, the optimization processes include aspects and parameters encompassing other layers of the stack, from channel models, signal energy, energy efficiency, throughput and connectivity/coverage requirements at physical layer [154, 155, 156, 157, 158, 159, 160], or resource scheduling at data link layer [161, 162, 157], and this justifies the cross-layer nature of these studies. Hence, no survey paper so far investigated these problems as a whole.

The Section closes up highlighting the lessons learnt on the theme. Figure 14 summarizes the overall organization of the present Section.

\subsection{Path Optimization and Collision Avoidance}

When discussing drones missions and task list, the specification of the main criteria for path design becomes of critical importance. Some of the most relevant problems related to path design and optimization criteria have been widely and extensively discussed in [11], [9], and [12]. Since those works are surveys, a deeper insight on the theme of optimizing the path a drone flights over has been discussed in many other works. For example, the problem of understanding which is the optimal trajectory has been studied in the context of UAV-enabled wireless 


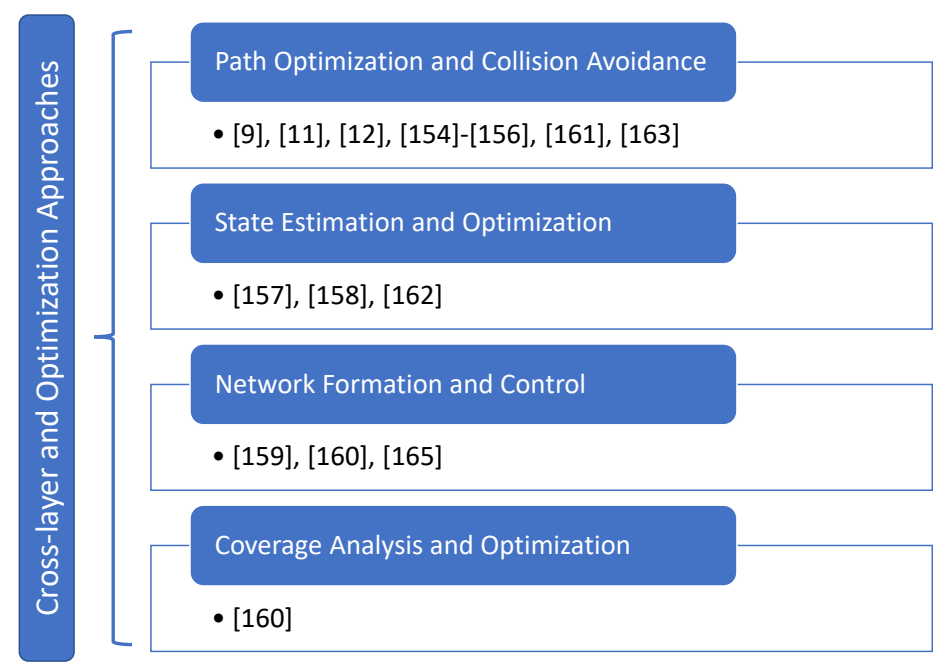

Figure 14: Cross-Layer and optimization section Taxonomy.

power transfer systems, as proposed in [154]. In particular, this study discusses the UAV maximum speed, which is generally constrained by physical layer aspects, even if the optimization occurs at application layer. This limitation leads to the design of complex hover-and-fly trajectories, which, still, deserve to be optimized. The proposal applies convex programming optimization technique to solve the problem.

Path optimization is a leading trend in research, since power and energy consumption are among the major stakeholders in UAVs characterization. On this theme, qualitative modeling for energy footprint optimization are widely discussed in several studies, such as [155, 156, 163], as associated to trajectories design. Those works are pretty similar since the whole set of parameters involved in all of them is almost the same. Still, they share the wider objective of finding out the minimum energy consumption, the bits allocation (i.e., for each task) and the shortest, yet most efficient, trajectories. Those parameters resulted to be simultaneously optimized. The proposed models are anyway in the need to be verified over wide swarms flying over long distances, while keeping coordinated flight conditions steady.

Another interesting work on the theme of trajectory design and path optimization is presented in [156]. It assumes that the UAV flies horizontally with a fixed altitude while exchanging data with a ground infrastructure. While on flight, the drones are supposed to communicate with the reference ground infrastructure in 
an energy-efficient way, that is granted thanks to the compliance to several KPIs, including the required throughput and the overall energy footprint. The work derives the contributions due to mechanical propulsion thanks to a theoretical model. The obtained framework is used to design multiple trajectories and iterates the application of the algorithm to demonstrate its versatility. The theme of complex trajectory design is discussed in [163] too. Here, the mission envisions frequent turnovers between hovering phases and altitude variations. Among the most relevant advantages of the proposed model there is its wide applicability to multicopters.

In [161] an energy-efficient scheme is proposed to include multiple variables for the optimization, such as the energy budget of each UAV, the number of tasks that each drone has to accomplish, and the type of data exchanged, together with the UAVs reference speed. Here, both uploading and downloading phases are handled relying on MEC solutions.

The study presented in [164] discusses the problem of hybrid delivery employing Truck-drones. in particular, this work frames an optimization problem related with the connection between the payload size and the energy spent while moving over certain trajectory. Moreover the proposed framework includes the possibility that No-Fly zones are within the mission plan. Even though the invasion scenario only include a single truck and a single drum, multiple vehicles are clearly among the future research perspectives. Even more so because the problem can be complicated with different scales or shapes of no fly zones, in which case fleet coordination becomes mandatory.

\subsection{State Estimation and Optimization}

Several works deal with the drones state estimation with the precise aim of identifying methods to minimize energy consumption, rather than designing trajectories or maximizing area covered during the mission.

The ability to communicate state information is discussed in [162], a proposal that aims at achieving the maximization of users throughput in a threedimensional space where dangerous conditions are verifying. The mathematical formulation of the problem include several parameters, such as overall throughput, remaining battery capacity for each UAV in a swarm, and several others. Those variables are modeled on a multi-period graph. Something similar is done in [157], a contribution that formulates an optimization problem to specifically address natural disaster scenarios in real-time conditions with relay-assisted UAVs.

State estimation represents the starting point of the study proposed in [158], in which multi-objective optimization algorithms are proposed. Their aim is to 
allocate tasks and plan paths for multiple UAVs forming a swarm. The proposal leverages the Genetic Algorithm (GA) approach to minimize the time in which the mission gets completed and includes area coverage and communication path. As a such, the problem also embraces network connectivity aspects. The solution can be fine-tuned to prioritize coverage or connectivity according to mission commitments.

\subsection{Network Formation and Control}

Two major survey contributions discussed network formation and controlrelated aspects in the context of the $\mathrm{IoD}[3]$ and [8]. A detailed analysis of those themes have been extensively discussed in the form of optimization problems in [165, 159, 160]. In particular, [165] discusses network formation as the result of an optimal task assignment problem. The formulation leverages the coordinated nature of a swarm of drones that are communicating through A2A links. Together with network formation thanks to the cooperation among multiple drones, efficient mission plans are constantly generated and updated to refine the obtained results.

Similarly, [159] proposes UAVs-enabled wireless communications between multiple ground nodes that are subjected to specific requirements, such as throughput, energy consumption optimization, and limitations on communication-related energy expenditure. The proposal models propulsion power in close correlation with physics-related phenomena. The model reaches the energy minimization objective as a results of a non-convex problem. Therefore, the preliminary flyhover-communicate assumption is empowered with a more sophisticated design criteria that includes the hovering location and duration, together with the flying trajectory design.

In the same field, another possibility is related to BS offloading and coverage area enhancement, as in 5G wireless communication systems [160]. Another interesting application is aided relaying, with UAVs providing reliable wireless connectivity between users or user groups in adverse environments.

\subsection{Coverage Analysis and Optimization}

An interesting research perspective is the BS offloading and coverage area enhancement, as in 5G wireless communication systems [160]. UAVs are often involved in information dissemination and/or data collection. In these cases, drones are arranged to fly over a certain area of interest and gather data. Here, aided relaying envisions UAVs providing reliable wireless connectivity between users or 
user groups in adverse environments. This use greatly reduces the overall energy consumption at the end-node level, thus extending the network lifetime.

\subsection{Lessons Learnt}

Many lessons can be learnt from the cross-layer approaches discussed in this section. All the topics presented (path and trajectory optimization and design, state estimation and optimization, and network formation and control) are of great importance to exchange data among UAV and/or between UAV and GS, while optimizing different metrics like energy consumption, connectivity, throughput, delay in data exchange, task accomplishment, overall mission time, connectivity, and coverage area. Especially the minimization of energy consumption pushes such optimization strategies, because of the positive implications on the increase of the flight time, battery duration and amount of data delivered to destination.

Nevertheless the metrics to be optimized are too many and often in contrast each other, so trade-offs have to be reached especially when the optimization strategies are implemented through multi-objective algorithms which are intrinsically much more computationally complex with respect to their single-objective counterparts. Very often, and as usual, simplifications are introduced to make these algorithms more computationally tractable, but with the risk of oversimplifying real application scenarios. Another weak point resides in the high mobility of drones, that brings to continuous and frequent changes in the network topology and connectivity. Accordingly, also the state information (position, velocity, etc.) must be continuously updated to recover from unavoidable mismatches between the estimated state and the real one, and this increases the overhead in exchange of control information among drones and/or with GSs.

\section{Security and Privacy Aspects}

This Section discusses security and privacy related topics that are of relevance in the context of the IoD. In Figure 15, the overall organization of the present Section is summarized.

UAVs can be considered as complex flight control computer-aided systems with, at least, one sensor. UAVs is programmed, off-line, before take off, or controlled, on-line, to fly over a specific pattern, for example within a facility. During this period of time, it is supposed to receive sensor data, thanks to on-board units, apply some processing routine to the sensor data, as to detect an unacceptable 


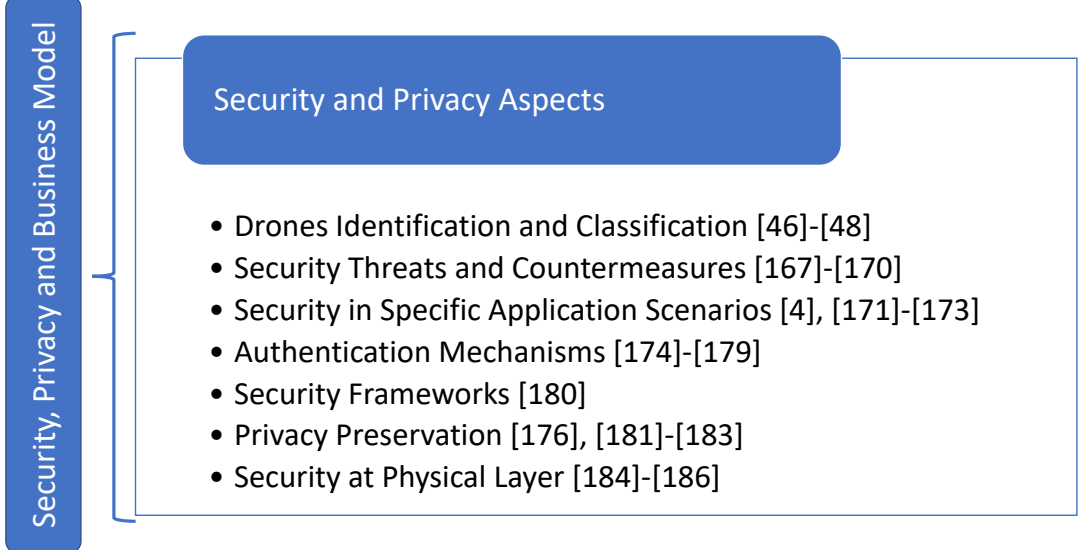

Figure 15: Security, privacy and business models Taxonomy.

level of difference in data features, determine a new flight instruction for the vehicle based on the processing. Afterward the new flight instructions are sent for controlling the flight of the vehicle.

According to the reference context, several security aspects may be involved. Conventionally, commercial surveillance systems are managed by humans, that regularly check in at guard stations and make observations. Surveillance systems have progressed into closed-circuit television monitoring, and more recently integrated systems have been developed to include video cameras installed at strategic locations in a facility. These video cameras are in communication with a centralized remote monitoring facility, and operators visually sweep the facility from the monitoring center. Upon discovery of suspicious activity, the operator can engage in a response plan. A surveillance solution has been employed by the military through surveillance unmanned aerial vehicles, commonly referred to as drones. Military surveillance drones are capable of flying over large areas such that video surveillance can be achieved. Military surveillance drones are very expensive and address surveillance of very large outdoor areas. However, they are not practical for businesses that want to maintain security at a single location or small group of locations such as a warehouse or a manufacturing facility.

In [166, 10], the leading theme is security in the context of the communications among drones. The main concern on the theme is related to the security and the privacy of the data exchange processes. 


\subsubsection{Drones Identification and Classification}

Modulation schemes are studied in some papers to identify and classify drones [46, 47, 48]. These contributions aim to identify drones on the basis of modulated RF signals [47, 48] and classify them based on their unique features [46]. The method proposed in [47] aims at preventing UAVs from unauthorized flights over reserved areas. The solution proposed to tackle this problem is to use SDN to process the UAVs control signals. On the basis of a detailed analysis of the UAVs control methods, that rely on different modulation techniques, this study proposes a simple detection technique through measurement campaigns that are conducted on the field. Two different commercially available UAVs are used to extract the main features of their control signals through a SDN. Another UAV detection technique is proposed in [48], for drones identification purposes. The paper carries out an analysis of the radio communication between the UAV and the remote control, as related to a specific type of drone. The radio communication channel is studied with reference to the number of channels adopted, their frequencies, the frequency utilization method and the modulation technique. This last aspect is used to study how the communication is maintained between the UAV and the remote controller through a signal analysis. A classification method for UAVs based on the analysis of the RF signal is proposed in [46], to avoid security and privacy issues and preserve public safety. Drone pilots are identified through the analysis of RF signals captured from the drone controllers, in presence of wireless co-channel interference, namely, WiFi and Bluetooth. The methodology adopted to detect the UAVs is first described, testifying the robustness against false alarms and missed target detection. The RF features are extracted to classify the controller signals of UAVs. A RF fingerprinting technique is proposed, able to capture the unique characteristics (the "fingerprint") of each UAV controller. The proposed approach is then compared with different Machine Learning algorithms, to testify its effectiveness for UAV classification.

\subsubsection{Security Threats and Countermeasures}

Some papers on security in drone networks are mainly focused on the description of the security threats and the related countermeasures in drones communication [167, 168, 169, 170]. The importance of cyber-security in UAV-based networks is highlighted in [167]. The main goal of this work is to describe the main security threats involving networks based on UAVs. To this end, the main challenges for networks of drones are first presented, among which the need to verify the identity of a drone in some application scenarios, proposing a classification of the drone based on its functionalities, range, altitude, endurance, etc. This 
classification is the starting point to describe the main attacks and security threats that can be carried out on drones, that testify how easily a UAV can be attacked, and its functionalities compromised. The network architecture is described, highlighting the main security threats as referred to each part of the network. Finally, a risk assessment scheme is proposed, that contains integrity, confidentiality and availability. The necessary steps to provide security to drones are considered as concluding considerations of this study. More focused on secure communications among drones is the study carried out in [168]. The security-related risks are addressed in this work, especially focusing on the "taking over control" type of attack. It is a software centric type of attack, and exploits the vulnerabilities of the standardized communication and control protocol. To this end, the possible approaches adopted to carry out attacks that exploit the protocol vulnerabilities are discussed in detail, also highlighting that they can be easily exported to other communication protocols. Then, some recommendations are made to improve the security of drones operations. They mainly consist in a strong limitation of the secret information disclosed during the binding phase, an extension of the binary string that represents the secret information exchanged, and the adoption of cryptographic methods, that are not actually present in the UAVs communication protocols. Other vulnerability issues are described in [169], with reference to a specific type of drone provided with video stream capabilities. This paper raises a warning flag from the privacy and resource accessibility points of view, which should absolutely not be set free in communication networks of any kind. This work focuses on the well known security vulnerabilities like an unencrypted Wi-Fi network connection and the management of the linux-based operating systems running on the drone by a user. This study also shows how the drone can be hacked, illustrating some possible attack scenarios that exploit the port scanning to carry out a combination of attacks that can take the full drone control. Then, some operations are described to secure the WiFi connection of the drone and its operation with the official smartphone app or third-party software. The work [170] discusses security of UAVs, but considering holistically the interactions among UAV systems from the point of view of attack methods and threats. The study is conducted from the perspective of a common security analysis among different systems, and the related security challenges to be tackled. The main goal is to derive a general security model, which can be exploited in different UAV-based systems, and that can analyze the security problems. The security analysis at the basis of the model is carried out by addressing both attacks at several layers of the stack, and potential threats, and analyzing security from the point of view of aerial, ground, marine and space unmanned systems. A summary of the security 
issues for the different systems paves the way to a discussion on the main security challenges for unmanned systems and the future directions that can be taken to tackle them.

\subsubsection{Security in Specific Application Scenarios}

Papers [171, 172, 173, 4] contextualize the UAV security analysis to specific application scenarios. The security aspect is addressed in [171], specifically referred to drone-assisted public safety networks. Drones can be fruitfully employed in public safety networks, where the supported services aim to protect people and environments from a wide variety of threats, because they enhance the communication capabilities of such wireless networks, since they can reduce coverage gaps and network congestion. Nevertheless, security is of great importance in this context, because sensitive data and/or critical information can be transmitted among drones. This contribution analyzes the most relevant security threats and the related countermeasures, that cover all the aspects of data confidentiality, integrity, availability, authenticity, privacy preservation and non-repudiation [171].

Another scenario, the smart farming environment, is considered in [172] to study security and privacy issues related to the use of IoT and communication technologies involving the use of UAVs, that are subject to cyber-security threats and vulnerabilities. This is mainly an overview paper, whose goal is first to provide an overall description of the communication architecture in the smart farming landscape, at different protocol layers. Then, the security and privacy issues are identified, in terms of security, privacy, authentication and authorization, and compliance with regulations. Cyber-attacks are then presented and classified into data, network, supply chain, and other relevant attacks. In parallel, the current research in cyber-security is discussed, describing also the main contributions and weak points of the works on this topic. Finally, the most interesting research challenges are addressed, that cover different areas ranging from network security to supply chain, artificial intelligence, machine learning, access control and information sharing [172].

Security plays a very important role also in military applications. For example, drones are widely used in the context of counter-terrorism [173]. Here, the concept of security is strictly related to the ability of inspecting active theaters of war. The conventional wisdom on drone warfare holds that drones are considered secure if, and perhaps only if, they have a high effectiveness in striking and disabling terrorist organizations. Nevertheless, the legitimacy of their employment is still under discussion. In fact, there is a diffused willingness to mitigate the strategic use of drones, at least under recognized standards and norms. 


\subsubsection{Authentication Mechanisms}

Authentication mechanisms in networks of UAVs are described in [174, 175, 176, 177, 178, 179]. The papers [174, 176, 178, 179] are entirely focused on authentication mechanisms in UAV-based networks. In particular, [174] leverages a mutual authentication protocol between UAVs and a ground station. This mechanism aims at overcoming the traditional cryptographic techniques which are inefficient in networks of UAVs, because of their tight resource constraints, together with the nature of the wireless medium that is "open" by its own nature. The proposed mechanism exploits the so-called Physical Unclonable Functions (PUFs), that are the unique physical identity of a device, that depends on its hardware characteristics. Based on PUFs, a challenge-response pair is unique, and is used to authenticate the UAV to the ground station, through the well known time-based one-time password algorithm. It is used to exchange the challenge-response pair between the UAV and the station in the initial phase of the proposed algorithm, that is developed in such a way to avoid an adversary to overhear, duplicate, corrupt, or alter authentication data. In a similar way to what previously discussed, the peculiarities of the IoD are taken into account by the work presented in [179], where data exchange between mobile users, a drone working a server and the remainder IoD infrastructure. Even though the operating context is similar, [178] focuses on an efficient privacy-aware scheme specifically designed for authenticated key agreement. The peculiarity of the contribution is that fact that no secret keys are store within the devices, still granting the desired security features.

Another discussion on authentication vulnerability in consumer WiFi drones is found in [175]. This work proposes a security assessment that illustrates how to manipulate a WiFi drone by a software suite, and without the need for additional hardware equipment that can carry out jamming or eavesdropping attacks, like for example a Software Defines Radio (SDR). The assessment procedure show that a deauthentication attack can be effectively carried out; it is a kind of Denial of Service (DoS) attack where a malicious entity launches deauthentication packets to a wireless access point (AP), making the AP believe that hey come from a real client, thus taking down the drone. The drone vulnerabilities are assessed by standardized assessment models. Some prevention strategies are also discussed, like the adoption of encryption protocols and best password practices. The work presented in [177] is an important contribution that tackles both privacy and security from a technological point of view, for example securing transmission and reception activities. More specifically, a mechanism for secure communications in UAV-based networks is proposed. It supports key agreement, user authentication, 
non-repudiation, and user revocation, and all this in one algorithm. This mechanism is then exploited to build a secure communication protocol for UAV-based applications, which aims at maximizing the energy efficiency. The performance of the proposed scheme is evaluated in a real testbed.

\subsubsection{Security Frameworks}

A security management framework is proposed in [180], which aims at automating the orchestration, configuration and deployment of lightweight Virtual network Security Functions (VSF) in Mobile Edge Computing (MEC) nodes, that are placed on-board UAVs. Different factors are considered to optimize the security orchestration. The contribution of this work is manyfold. First, a MEC architecture is proposed and implemented. With this background, the orchestration mechanism is provided, to manage security in UAV networks, and taking into account different metrics and conditions for the VNF placement on UAVs, like operating capacity, battery, atmospheric conditions, computing resources (RAM, disk, CPU), and network metrics. The applicability of the proposed solution is then evaluated in a real testbed and use case, testing the performance of the framework from both the MEC node and the UAV points of view.

\subsubsection{Privacy Preservation}

Privacy is also a critical issue in the IoD environment, as described in [181, 176, 182, 183]. This aspect is developed in [181]. This paper discusses the security challenges from the legal point of view, starting from the assumption that the legal framework for UAVs is very complex in the EU scenario. The main goal of this paper is to point out the key open issues in the legal field, that include public security legislations, telecommunication law, rules on product liability, criminal and insurance law, privacy and data protection. An overview of the new EU rules is first provided, describing the approach followed by the EU to guarantee security and safety for aircraft operations in the sky. The open issues on the security topic are then addressed, synthesized into privacy preservation, telecommunications and cyber-security breaches, registration of UAVs and the identifiability of their operators and pilots, liability and enforcement regulations. The resulting picture confirm the complexity of the legal framework that necessitates harmonization and clarification of rules [181].

A major privacy concern is directly linked to the idea that drones are moving all around urban and suburban areas, impairing privacy of the people, especially in case of amateur drones. This is the theme developed in [176], where different safety, security and privacy threats are discussed in the deployment of amateur 
UAVs. The main goal of this review paper is to illustrate the state-of-the-art studies on this topic. A framework is then proposed, that exploits cognitive IoT to provide amateur drone surveillance with a higher level of intelligence through cognitive tasks, i.e., sensing, data analytics, knowledge discovery and intelligent decision making [176]. A case study is also provided, where detection of both authorized and unauthorized drones is provided, to check the correct operations that the various types of drones can carry out.

The contribution [182] specifically addresses privacy issues of IoD in both civilian and military architectures and a certain number of security and privacy requirements. The main contribution of this paper is to study the IoD architecture and its main security and privacy requirements. To this end, the main security and privacy challenges in the IoD scenario are described, that range from the privacy leakage, that occurs when sensitive data are collected by unauthorized drones, to data sharing in the cloud that can be readable by third-parties, and malicious interference in control sites that occurs whenever a drone can be managed, controlled in all its movements by an attacker to compromise a system. For all these issues, solutions are proposed aiming at protecting the privacy of sensitive data. They consist of authentication solution for privacy protection to lightweight cryptography protocols for security and privacy protection in case of stored data. A lightweight data protection protocol is also proposed for data stored in a cloud server. It is based on the well-known Identity-Based Encryption (IBE) protocol, but it is made lightweight to adapt it to the resource-constrained drones. Finally, future research directions are also suggested to tackle the main security challenges explained above.

The use of drones drones is widely used also in commercial applications, and this raises concerns about the related privacy and safety issues arising from a domestic employment of drones. This is the main goal of the work [183], that carries out an exhaustive review of the state-of-the-art literature on drones thought for commercial uses, but focusing on the so-called "tecnoethical" aspects, i.e., the study of the impact of drones technology on ethics. Social and ethical concerns are studied in different key-areas, also providing insights on the state of the public knowledge on commercial drones, but seen from an ethical point of view. Finally, this paper points out also the need to increase the level of attention towards the potential negative ethical consequences in the use of commercial drones.

\subsubsection{Security at Physical Layer}

Some papers tackle the security aspect in UAV networks from the physical layer perspective [184, 185, 186]. From this point of view, an interesting research 
topic about security is jamming, as testified in [184, 185]. In [184] jamming is discussed as a kind of attack in optical UAV network architectures. The jamming attack is presented as a kind of Denial of service (DoS) attack, where the attacker sends intentionally and interference signal in the communication link between UAVs exploiting the Free Space Optical (FSO) link. A technique for jamming attack detection is proposed, able to detect the presence of jamming signals in combination with the transmitter signals. A countermeasure approach is also presented, allowing to recover the transmitted signals in presence of a jamming attack. Another solution to the problem of jamming is presented in [185] for UAV-aided cellular systems. This paper proposes a UAV relay scheme based on the Reinforcement Learning (RL) approach, that exploits the RL and transfer learning to optimize the relay signal power against jamming, without knowing the network topology and the models of message generation and jamming signal. The proposed scheme estimates the received jamming power, the condition of the links between jammer, UAV and the base stations. From these estimations, the UAV chooses the best relay policy that improves the network resistance towards jamming attacks. neural network is exploited to learn from past UAV relay experiences in similar cellular anti-jamming communication systems. Performance bounds, including bit error rate and computational complexity of the proposed relay scheme, are derived through simulation. A more general description of physical layer related security is carried out in [186], where eavesdropping and jamming attacks are taken into account in wireless networks including UAVs. Eavesdropping and jamming attacks are first described from two different points of view. In the first, UAV-ground communications are analyzed as more subject than terrestrial communications to eavesdropping and jamming attacks by malicious ground nodes. in the second, malicious UAVs can be more effective in carrying out eavesdropping and jamming attacks to terrestrial communications, if compared to malicious ground nodes. Starting from these premises, countermeasures are proposed to secure A2G communications from terrestrial eavesdropping and jamming, and to secure terrestrial communications from UAV eavesdropping and jamming.

\subsection{Lessons Learnt}

In the military context, both the role and employment are under discussion nowadays, since a regulation is highly wished from many players. Turning from the military to the civil context, the considerations connected with privacy are due to lack of legislation. Sometimes, the problems are related to the fact that not all the countries are using same regulations and/or laws on the theme. 


\section{Discussion on the Main Findings and Future Research Perspectives}

The aim of this Section is to discuss the details of the main findings of the analysis carried out so far. At the same time, in this part, the present contribution is specifically focused on the main lessons learnt from the surveyed literature. Particular attention is devoted to the strengths of the main results found and the related issues.

\subsection{Discussion on the Main Findings in the Surveyed Literature}

The analysis of UAVs connectivity demonstrates an extremely wide set of possible technologies involved for creating both $\mathrm{A} 2 \mathrm{~A}$ and $\mathrm{A} 2 \mathrm{G}$ links. In terms of channel modeling and connectivity analysis, several studies show that there is a tight bound between the two aspects. Several proposals aim at creating reliable mathematical formulations that include as much aspects as possible, in terms of statistical variability and signal fluctuations in the communication channel. They are very useful to analyze the increase in coverage performance, the optimal allocation of resources at different layers of the protocol stack, and the improvements in path reliability for routing, path planning and position optimization strategies. Although the presented contributions are valuable, connectivity and channel models suffer from multiple problems, mainly connected to real-world operating conditions, such as NLoS conditions and/or presence of obstacles. To solve such issues, the most promising solution seems to be the choice of the most suitable communication technology, that matches the specific application needs.

At data link layer, the current state of the art suggests that drones may benefit from significant modifications to MAC layer. For example, cooperative schemes are useful to improve communication efficiency. Synchronization is another important point that arises from the analysis carried out. To reach optimal resource allocation, scheduling is widely considered as the most promising strategy, especially in high densely populated scenarios in which drones are configured as swarms.

Network layer-related problems are, once again, strictly related to enhancements in connectivity; drones are proposed as relaying units to strengthen existing networking solutions.

Turning to application layer considerations, the solutions that have been investigated so far demonstrate that applications may strongly benefit from the adoption of cooperation among drones, as suggested in lower layers solutions. Some of the most interesting works show the suitability of drones for advanced applications in continuous monitoring of widely distributed phenomena, surveillance among 
others. What emerges as a clear perspective of the employment of drones is the fact that the inspection of difficult access areas is sensibly simplified. Almost all of the referenced works on this topic agree that the quick on-site deployment of inspection system composed by one or more drones generates crucial benefits.

The analysis and optimization of throughput has been discussed as a way to increase the performance of communication systems in the context if both A2A and A2G links for data exchange, and to optimize different metrics in IoD systems (mutual distance, positions and paths of drones, spectrum and energy efficiency, network topologies). In such context, the throughput optimization in most cases translates into solving an optimization problem, whose solution is computationally expensive, or found approximately, or only in simplified scenarios. Furthermore, throughput maximization is counterbalanced by a higher energy consumption, which can become a serious drawback for battery-powered drones. Given the formulations of the optimization problems proposed for resource allocation, the currently available technological landscape seems to be a sensibly limiting factor, in terms of onboard energy availability and computational capabilities.

Despite the wide agreement on the fact that drones may sensibly enhance all the processes in which they may be involved, some legal issues are clearly arising. In fact, there is too much lack of legislation in many countries to allow a massive employment of networks of drones as enabling technology. Moreover, some of the biggest players in this market argue that legislation should be somehow homogeneous across borders, thus allowing industrial players to act on a worldwide scale.

\subsection{Research Challenges and Possible Future Directions}

In this section, the main research challenges and possible future directions in the IoD field are discussed.

As for communications among drones, the main trends are related to the extension of the covered area and the employability of communication protocols to guarantee a target QoE in both $\mathrm{A} 2 \mathrm{~A}$ and $\mathrm{A} 2 \mathrm{G}$. In the $5 \mathrm{G}$ perspective, it could be useful to standardize communications and creating homogeneous frameworks and platforms that provide real integration among heterogeneous technologies, rather than pushing on performance (e.g., increasing the data rate or decreasing latencies). This process is often referred to as Softwarization. It is worth noting that a similar phenomenon is taking place in the IoT world., where a number of examples and dedicated studies are specifically focused at integrating the existing IoT technologies rather than developing new ones [187, 188, 189, 190]. Specific attention is devoted to the security aspects of such interactions [191], thus proposing 
innovative solutions based on Blockchain and smart contracts [192].

Another example can be found in the application of VLCs and ICN technologies to drones. Some works have already contributed on the VLC topic $[87,193,194,89,88,90,195,196,197]$, especially for what concerns the analysis of the IEEE standards [87], modulation schema and light patterns at physical layer [194], MAC layer analysis and performance of network topologies [89, 88], simulation software [88, 90], applications of the VLC system architecture [195, 197] with its strengths and weaknesses [196], and related performance evaluation [193]. It would be very interesting, as a future research direction, to investigate the possibilities of application of this novel technology to the IoD field.

Another research challenge in drone communication concerns security and privacy in data exchange. Strictly related to privacy is the legislation issue. Legislative interventions could also be of importance on the theme of privacy, even if some problems have still to be solved [176]. In particular, one of the concerns is related to the idea that drones moving all along urban and suburban areas could impair privacy of the people. To the best of the authors knowledge, very few works analyze these aspects: the work [177] treats these themes from a technological point of view, for example securing transmission and reception activities. The contribution [182] specifically addresses privacy issues of the IoD in both civilian and military architectures and a certain number of security and privacy requirements. The work [169] raises a flag from the privacy and resource accessibility points of view, which should absolutely not be set free in communication networks of any kind. Nevertheless, research efforts could be made in this direction to further analyze and improve these aspects.

Another interesting research line that definitely deserves attention is the application of ICN in the context of the IoD [198]. In particular, what clearly emerges from the study of the related state-of-the-art, is that the application of the ICN in the context of ITSs seems not to be already as mature as it could [199, 200]. As a matter of fact, the authors believe that this may represent a limiting factor since the ICN architecture proposes one of the best solutions to support communications over users, especially in mobile-by-design scenarios [201, 202]. Among the main reasons that make this of relevance, there is the increasingly emerging limitation of the host-centric nature of the Internet, as known today. Indeed, there is a continuous request for seamless mobility, coming from users of different nature, and pushing to the limit the Internet capabilities. The proper support to intrinsic mobile scenarios becomes necessary in those cases in which the handover is frequently verified. The latter is, in fact, an extremely well-known problem in the telecommunications world as, in a mobile scenario, the user remains connected 
to the network, and does not experience any discontinuity of service, if, and only if, the coverage is acceptable. Since the global connectivity cannot be granted by using a single access point (i.e., a single cell in the case of cellular networks), the problem of the handover arises when the user moves from one access point to another. In the case of cellular networks, this happens when the user moves from one cell to one of the neighboring ones. The intrinsic mobility that characterizes ITSs systems, both from the points of view of users and drones producing data, places serious constraints on sustainability. It is worth noting that routing problems also arise in these cases, because of the need to realize in real-time which access point is the UAV connected to, and both in DL and UL. If the routes are not known, the UAV will experience a loss of connectivity and information exchange. The ICN communication paradigm leveraging the Publish-Subscribe communication scheme represents a good candidate for granting QoS design criteria, for instance communication latencies, PLR, resilience, and throughput. The joint analysis of even some of these aspects, combined together in ITS applications, could be an interesting challenge for the future research.

\subsection{Other Remarks}

Some other considerations are related to technology and to the massive production and widespread diffusion of autonomous vehicles, even more so in industrial applications. It is well known that there is a close relationship between costs and the development of a technology [203]. In this context, advanced industrial countries select, and apply without costs, all the innovations that are more immediately useful. As the general level of capital accumulation rises, more capitalintensive technologies become economical. Since industrialization is driven by technology applicability, and considerably boosted by rapid prototyping, the need for a drone to carry out part of industrial process monitoring or supply chain support/optimization depends on its cost and its accessibility. The consideration can be completed by reasoning on the legal aspects of the question. Since there is not a unified legislation on the usage of unmanned vehicles, the seamless employability is still far from now. In a global economical context, this means that a firm or an industry may find economical and legal barriers to their welcoming attitude towards drones. In all the analyzed works, these aspects have been neglected, but they surely represent a value added in future research on the IoD theme. 


\section{Conclusions}

This work aims at providing a thorough overview on the research activities on the IoD network architecture. To this end, the available scientific literature has been studied in detail and classified in order to identify all the current research trends.

At a first level of analysis, the proposed classification scheme follows the Internet protocol stack, starting from the physical layer, going up toward the application layer, without neglecting cross-layer approaches. At a finer description level, for each layer of the stack the papers have been further classified and described, based on the approach proposed.

While describing the challenges that threaten the IoD diffusion, this work also presents the current open issues, in order to draw future research directions. The analysis demonstrates that consistent design efforts have been devoted to specific aspects at the different layers. For instance, several mathematical models/frameworks have been proposed to improve communication range, or reliably model the wireless medium, at physical layer, or even efficiently routing for synchronization. Some works propose cooperation and network formation as an efficient data exchange strategy.

Promising results have been discussed in those works that focus on one of the aforedescribed problems and try to deal with it as an optimization problem. This is even more evident when the IoD-related problems are solved together in combined optimization approaches, such as energy consumption and trajectory design, or path planning and routing optimization.

At the same time, novel proposals and design methodologies were surveyed to discuss the effects of the introduction of $5 \mathrm{G}$ and $6 \mathrm{G}$-compliant technologies, such as mmWave and VLC. Even though the discussion is still ongoing, further efforts could be useful to establish new requirements even in critical operating conditions.

\section{List of Acronyms}

$6 \mathrm{G}$

Sixth-Generation

610 IPv6 over networks of resource-constrained nodes

6LoWPAN IPv6 over Low power Wireless Personal Area Networks

6TiSCH IPv6 over the TSCH mode of IEEE 802.15.4 


\begin{tabular}{|c|c|}
\hline 6top & 6tisch Operation Sublayer \\
\hline $\mathrm{A} 2 \mathrm{~A}$ & Air-to-Air \\
\hline $\mathrm{A} 2 \mathrm{G}$ & Air-to-Ground \\
\hline ACODS & Adaptive Computation Offloading Drone System \\
\hline AGV & Automated Guided Vehicle \\
\hline AI & Artificial Intelligence \\
\hline AODV & Ad-hoc On-demand Distance Vector \\
\hline AP & Allocation Policy \\
\hline $\mathrm{AQ}$ & Autonomous Quadcopter \\
\hline ASAP & As-Soon-As-Possible Schedule Another Parent \\
\hline ASN & Absolute Slot Number \\
\hline ASV & Autonomous Surface Vehicle \\
\hline AUV & Autonomous Underwater Vehicle \\
\hline BDMA & Beam-Division Multiple Access \\
\hline $\mathrm{BS}$ & Base Station \\
\hline $\mathrm{CCN}$ & Content-Centric Network \\
\hline CCTV & Closed Circuit TeleVision \\
\hline CEA & Cell Estimation Algorithm \\
\hline $\mathrm{CH}$ & Cluster Head \\
\hline CIR & Channel Impulse Response \\
\hline CoAP & Constrained Application Protocol \\
\hline CoRE & Constrained RESTful Environments \\
\hline CR & Cognitive Radio \\
\hline
\end{tabular}




\begin{tabular}{|c|c|}
\hline CSMA & Carrier Sense Multiple Access \\
\hline CSMA/CA & Carrier Sense Multiple Access with Collision Avoidance \\
\hline CTD & Conductivity, Temperature, Depth \\
\hline D2D & Device-to-Device \\
\hline DAG & Directed Acyclic Graph \\
\hline DAO & Destination Advertisement Object \\
\hline DETB & Dynamic Energy \& Traffic Balance \\
\hline DGAC & Direction Générale de l'Aviation Civile \\
\hline $\mathrm{DIO}$ & Destination Information Object \\
\hline DL & DownLink \\
\hline DNS & Domain Name System \\
\hline DODAG & Destination Oriented DAG \\
\hline DP & Dynamic Programming \\
\hline DRL & Deep Reinforcement Learning \\
\hline DSSS & Direct Sequence Spread Spectrum \\
\hline E2E & End-to-End \\
\hline EASA & European Aviation Safety Agency \\
\hline ENAC & Ente Nazionale per l'Aviazione Civile \\
\hline $\mathrm{EOF}$ & End Of Frame \\
\hline ePFC & extended Potential Field Controller \\
\hline FAA & Federal Aviation Administration \\
\hline FANET & Flying Ad-hoc NETwork \\
\hline FBS & Flying Base Station \\
\hline
\end{tabular}


FDM

FDMA

FFD

FIR

FoV

FSO

G2G

GA

GDA

GIS

GPS

GPSR

GS

HTTP

ICI

ICN

ICT

IDFT

IETF

IIoT

IMC

IMU

IoD
Frequency Division Multiplexing

Frequency Division Multiple Access

Fully Functioned Device

Finite Impulse Response

Field-of-View

Free Space Optical

Ground-to-Ground

Genetic Algorithm

Gradient Algorithm

Geographic Information System

Global Position System

Greedy Perimeter Stateless Routing

Ground Station

HyperText Transfer Protocol

Inter-Carrier Interference

Information-Centric Networking

Information and Communication Technologies

Inverse Discrete Fourier Transform

Internet Engineering Task Force

Industrial Internet of Things

Internal Model Control

Inertial Measurement Unit

Internet of Drones 


\begin{tabular}{|c|c|}
\hline IoT & Internet of Things \\
\hline IP & Internet Protocol \\
\hline IPv4 & Internet Protocol version 4 \\
\hline IPv6 & Internet Protocol version 6 \\
\hline ISM & Industrial, Scientific and Medical \\
\hline ISP & Internet Service Provider \\
\hline ITS & Intelligent Transportation System \\
\hline JSON & JavaScript Object Notation \\
\hline KPI & Key Performance Index \\
\hline KPIs & Key Performance Indices \\
\hline LA & Learning Algorithm \\
\hline LAR & Location-Aided Routing \\
\hline LDRA & Low Delay Routing Algorithm \\
\hline LLN & Low-power Lossy Network \\
\hline LLSF & Low-power Lossy Network \\
\hline LoRaWAN & Long Range Wide Area Network \\
\hline LoS & Line of Sight \\
\hline LPWAN & Low Power Wide Area Network \\
\hline LTE & Long-Term Evolution \\
\hline LTE-A & Long-Term Evolution-Advanced \\
\hline LTE-BS & Long-Term Evolution Base Station \\
\hline LTE-U & Long-Term Evolution - Unlicensed \\
\hline M2M & Machine-to-Machine \\
\hline
\end{tabular}




\begin{tabular}{|c|c|}
\hline MA & Multiple Access \\
\hline MAC & Medium Access Control \\
\hline MANET & Mobile Ad-hoc NETwork \\
\hline $\mathrm{MCU}$ & Micro-Controller Unit \\
\hline MEC & Mobile Edge Computing \\
\hline MINLP & Mixed Integer Non-Linear Programming \\
\hline MPTCP & Multipath TCP \\
\hline MSPRT & Multi Sequential Probability Ratio Test \\
\hline MTC & Machine-Type Communication \\
\hline MTC & mission critical Machine-Type Communication \\
\hline MTU & Maximum Transmission Unit \\
\hline $\mathrm{NC}$ & Network Coding \\
\hline NFP & Networked Flying Platform \\
\hline NFV & Network Function Virtualization \\
\hline NLoS & Non-Line of Sight \\
\hline NOMA & Non-Orthogonal Multiple Access \\
\hline O-QPSK & Offset-Quadrature Phase-Shift Keying \\
\hline OFDM & Orthogonal Frequency-Division Multiplexing \\
\hline OLSR & Optimized Link State Routing Protocol \\
\hline OS & Operating System \\
\hline OTF & On The Fly \\
\hline $\mathrm{P} 2 \mathrm{P}$ & Peer-to-Peer \\
\hline PCC & Path Computation Client \\
\hline
\end{tabular}




\begin{tabular}{|c|c|}
\hline PCE & Path Computation Element \\
\hline PCEP & Path Computation Element Protocol \\
\hline PDR & Packet Delivery Ratio \\
\hline PHY & Physical Layer \\
\hline PID & Proportional-Integral-Derivative \\
\hline PLA & PolyLactic Acid \\
\hline PLR & Packet Loss Ratio \\
\hline PSH & Problem Specific Heuristic \\
\hline PSO & Particle Swarm Optimization \\
\hline PxIMU & Pixhawk Inertial Measurement Unit \\
\hline QoE & Quality of Experience \\
\hline QoL & Quality of Link \\
\hline QoS & Quality of Service \\
\hline RFD & Reduced Functioned Device \\
\hline RMM & Random Mobility Model \\
\hline RMS-DS & Root Mean Square Delay Spread \\
\hline ROLL & Routing Over Low power and Lossy networks \\
\hline ROS & Robot Operating System \\
\hline ROV & Remotely Operated underwater Vehicle \\
\hline RPL & Routing Protocol for Low-power and Lossy networks \\
\hline RSMA & Rate-Splitting Multiple Access \\
\hline RSS & Received Signal Strength \\
\hline RSSI & Received Signal Strength Indication \\
\hline
\end{tabular}




\begin{tabular}{ll} 
RSU & RoadSide Unit \\
RTT & Round Trip Time \\
SAIN & Space-Air Integrated Network \\
SC-FDM & Single Carrier - Frequency Division Multiplexing \\
SDMA & Spatial-Division Multiple Access \\
SDN & Software Defined Network \\
SDR & Software Defines Radio \\
SE & Spectral Efficiency \\
SF0 & Scheduling Function Zero \\
SFD & Start of Frame Delimiter \\
SINR & Signal to Interference-plus-Noise Ratio \\
SLAM & Simultaneous Localization And Mapping \\
SNR & Signal-to-Noise Ratio \\
SPI & Serial Peripheral Interface \\
TCP & Transmission Control Protocol \\
TDMA & Time Division Multiple Access \\
TI & Texas Instruments \\
TSCH & Time Slotted Channel Hopping \\
UAS & Unmanned Aircraft System \\
UASN & Underwater Acoustic Sensor Network \\
UAV & Unmanned Aerial Vehicle \\
UAV-BS & Unmanned Aerial Vehicle Base Station \\
UDP & User Datagram Protocol \\
\hline
\end{tabular}




$\begin{array}{ll}\text { UE } & \text { User Equipment } \\ \text { UFP } & \text { Unmanned Flying Platform } \\ \text { UGV } & \text { Unmanned Ground Vehicle } \\ \text { UL } & \text { UpLink } \\ \text { USB } & \text { Universal Serial Bus } \\ \text { USV } & \text { Unmanned Surface Vehicle } \\ \text { UTM } & \text { Unmanned Aerial System Traffic Management } \\ \text { UUV } & \text { Unmanned Underwater Vehicle } \\ \text { UWSN } & \text { Underwater Wireless Sensor Network } \\ \text { V2I } & \text { Vehicle-to-Infrastructure } \\ \text { V2V } & \text { Vehicle-to-Vehicle } \\ \text { VANET } & \text { Vehicular Ad-hoc NETwork } \\ \text { VLC } & \text { Visible Light Communication } \\ \text { VMS } & \text { Variable Message Sign } \\ \text { WG } & \text { Working Group } \\ \text { WSN } & \text { Wireless Sensor Network }\end{array}$

\section{References}

[1] M. Gharibi, R. Boutaba, S. L. Waslander, Internet of drones, IEEE Access 4 (2016).

[2] A. Kumar, P. L. Mehta, Internet of drones: An engaging platform for iiot-oriented airborne sensors, in: Smart Sensors for Industrial Internet of Things, Springer, 2021, pp. 249-270.

[3] V. Sharma, R. Kumar, Cooperative frameworks and network models for flying ad hoc networks: a survey, Concurrency and Computation: Practice And Experience 29 (4) (2017) 1-36. 
[4] A. Nayyar, B.-L. Nguyen, N. G. Nguyen, The internet of drone things (iodt): Future envision of smart drones, in: First International Conference on Sustainable Technologies for Computational Intelligence, Springer, 2020, pp. 563-580.

[5] A. A. Khuwaja, Y. Chen, N. Zhao, M. S. Alouini, P. Dobbins, A survey of channel modeling for uav communications, IEEE Communications Surveys Tutorials (2018) 1-1doi:10.1109/COMST.2018.2856587.

[6] A. Fotouhi, H. Qiang, M. Ding, M. Hassan, L. G. Giordano, A. GarciaRodriguez, J. Yuan, Survey on UAV Cellular Communications: Practical Aspects, Standardization Advancements, Regulation, and Security Challenges, IEEE Communications Surveys \& Tutorials PP (c) (2018) 1.

[7] G. Iacovelli, P. Boccadoro, L. Grieco, An iterative stochastic approach to constrained drones' communications, in: Proc. of IEEE/ACM 24th International Symposium on Distributed Simulation and Real Time Applications (DS-RT) (DS-RT'20), Prague, Czech Republic, 2020.

[8] L. Gupta, R. Jain, G. Vaszkun, Survey of Important Issues in UAV Communication Networks, IEEE Communications Surveys and Tutorials 18 (2) (2016) 1123-1152. doi:10.1109/COMST.2015.2495297.

[9] Y. Liu, F. Wu, J. Wu, Cellular uav-to-device communications: Joint trajectory, speed, and power optimisation, IET Communications (2021).

[10] G. Choudhary, V. Sharma, T. Gupta, J. Kim, I. You, Internet of drones (iod): Threats, vulnerability, and security perspectives, arXiv preprint arXiv:1808.00203 (2018).

[11] N. H. Motlagh, T. Taleb, O. Arouk, Low-altitude unmanned aerial vehiclesbased internet of things services: Comprehensive survey and future perspectives, IEEE Internet of Things Journal 3 (6) (2016) 899-922.

[12] Y. Chen, H. Zhang, M. Xu, The coverage problem in uav network: A survey, in: Fifth International Conference on Computing, Communications and Networking Technologies (ICCCNT), 2014, pp. 1-5. doi: $10.1109 /$ ICCCNT.2014.6963085

[13] G. Ferri, A. Munafò, A. Tesei, P. Braca, F. Meyer, K. Pelekanakis, R. Petroccia, J. Alves, C. Strode, K. LePage, Cooperative robotic networks 
for underwater surveillance: an overview, IET Radar, Sonar \& Navigation 11 (12) (2017) 1740-1761.

[14] C. T. Cicek, H. Gultekin, B. Tavli, H. Yanikomeroglu, UAV Base Station Location Optimization for Next Generation Wireless Networks: Overview and Future Research Directions, CoRR abs/1812.11826 (2018). arXiv: 1812.11826 .

URL http://arxiv.org/abs/1812.11826

[15] C. Zhang, W. Zhang, W. Wang, L. Yang, W. Zhang, Research challenges and opportunities of uav millimeter-wave communications, IEEE Wireless Communications 26 (1) (2019) 58-62. doi:10.1109/MWC . 2018 . 1800214 .

[16] A. Alnoman, A. Anpalagan, On d2d communications for public safety applications, in: 2017 IEEE Canada International Humanitarian Technology Conference (IHTC), 2017, pp. 124-127.

[17] M. Alzenad, M. Z. Shakir, H. Yanikomeroglu, M. Alouini, Fso-based vertical backhaul/fronthaul framework for $5 \mathrm{~g}+$ wireless networks, IEEE Communications Magazine 56 (1) (2018) 218-224.

[18] A. Bader, M.-S. Alouini, An ultra-low-latency geo-routing scheme for team-based unmanned vehicular applications, in: 2015 IEEE Global Communications Conference (GLOBECOM), 2015, pp. 1-6.

[19] S. Colonnese, A. Carlesimo, L. Brigato, F. Cuomo, Qoe-aware uav flight path design for mobile video streaming in hetnet, in: 2018 IEEE 10th Sensor Array and Multichannel Signal Processing Workshop (SAM), 2018, pp. 301-305. doi:10.1109/SAM.2018.8448608.

[20] D. G. Cileo, N. Sharma, M. Magarini, Coverage, capacity and interference analysis for an aerial base station in different environments, in: 2017 International Symposium on Wireless Communication Systems (ISWCS), 2017, pp. 281-286.

[21] L. Ferranti, F. Cuomo, S. Colonnese, T. Melodia, Drone cellular networks: Enhancing the quality of experience of video streaming applications, Ad Hoc Networks 78 (2018) 1 - 12. doi:https://doi.org/10.1016/ j.adhoc.2018.05.003 
URL http://www.sciencedirect.com/science/article/ pii/S157087051830204X

[22] A. Koubâa, B. Qureshi, M.-F. Sriti, A. Allouch, Y. Javed, M. Alajlan, O. Cheikhrouhou, M. Khalgui, E. Tovar, Dronemap planner: A serviceoriented cloud-based management system for the internet-of-drones, Ad Hoc Networks 86 (2019) 46 - 62. doi:https://doi.org/10. $1016 / j \cdot a d h o c .2018 .09 .013$.

[23] H. Menouar, I. Guvenc, K. Akkaya, A. S. Uluagac, A. Kadri, A. Tuncer, Uav-enabled intelligent transportation systems for the smart city: Applications and challenges, IEEE Communications Magazine 55 (3) (2017) 2228.

[24] W. Saad, M. Bennis, M. Chen, A vision of 6g wireless systems: Applications, trends, technologies, and open research problems, IEEE network 34 (3) (2019) 134-142.

[25] D. Vasiliev, A. Chunaev, A. Abilov, I. Kaysina, D. Meitis, Application layer arq and network coding for qos improving in uav-assisted networks, in: 2019 25th Conference of Open Innovations Association (FRUCT), 2019, pp. 353-360.

[26] B. Van den Bergh, A. Chiumento, S. Pollin, Ultra-reliable ieee 802.11 for uav video streaming: From network to application, in: R. El-Azouzi, D. S. Menasche, E. Sabir, F. De Pellegrini, M. Benjillali (Eds.), Advances in Ubiquitous Networking 2, Springer Singapore, Singapore, 2017, pp. 637647.

[27] T. Zahariadis, A. Voulkidis, P. Karkazis, P. Trakadas, Preventive maintenance of critical infrastructures using 5g networks drones, in: 2017 14th IEEE International Conference on Advanced Video and Signal Based Surveillance (AVSS), 2017, pp. 1-4. doi:10.1109/AVSS.2017. 8078465 .

[28] X. Wang, A. Chowdhery, M. Chiang, Networked drone cameras for sports streaming, in: 2017 IEEE 37th International Conference on Distributed Computing Systems (ICDCS), 2017, pp. 308-318.

[29] A survey of open-source uav flight controllers and flight simulators, Microprocessors and Microsystems 61 (2018) $11-20$. 
[30] B. D. Deebak, F. Al-Turjman, Drone of IoT in 6G Wireless Communications: Technology, Challenges, and Future Aspects, Springer International Publishing, Cham, 2020, pp. 153-165. doi: $\{\$ 10.1007 /$ 978-3-030-38712-9_9\$\}.

[31] L. Bariah, L. Mohjazi, S. Muhaidat, P. C. Sofotasios, G. K. Kurt, H. Yanikomeroglu, O. A. Dobre, A prospective look: Key enabling technologies, applications and open research topics in 6g networks, IEEE Access 8 (2020) 174792-174820. doi:10.1109/ACCESS.2020. 3019590

[32] B. Deebak, F. Al-Turjman, Drone of iot in $6 \mathrm{~g}$ wireless communications: Technology, challenges, and future aspects, in: Unmanned Aerial Vehicles in Smart Cities, Springer, 2020, pp. 153-165.

[33] W. Saad, M. Bennis, M. Chen, A vision of $6 \mathrm{~g}$ wireless systems: Applications, trends, technologies, and open research problems, IEEE Network 34 (3) (2020) 134-142. doi:10.1109/MNET.001.1900287.

[34] P. Yang, Y. Xiao, M. Xiao, S. Li, 6g wireless communications: Vision and potential techniques, IEEE Network 33 (4) (2019) 70-75. doi:10. 1109/MNET.2019.1800418.

[35] Business Insider Intelligence, The future of drones for consumers, businesses, and the military, DRONES 101 (4 2018).

[36] S. Pollin, E. Vinogradov, Tut-23: Ieee icc tutorial on wireless communications with unmanned aerial vehicles, in: IEEE International Conference on Communications, 2019.

[37] A. Castillo O'Sullivan, A. D. Thierer, Projecting the growth and economic impact of the internet of things, Available at SSRN 2618794 (2015).

[38] J. Koiwanit, Analysis of environmental impacts of drone delivery on an online shopping system, Advances in Climate Change Research 9 (3) (2018) 201-207.

[39] L. M. PytlikZillig, B. Duncan, S. Elbaum, C. Detweiler, A drone by any other name: Purposes, end-user trustworthiness, and framing, but not terminology, affect public support for drones, IEEE Technology and Society Magazine 37 (1) (2018) 80-91. doi:10.1109/MTS.2018.2795121. 
[40] D. Jenkins, B. Vasigh, The economic impact of unmanned aircraft systems integration in the united states. arlington, va: Association of unmanned vehicle systems international, International, 2013.

[41] T. Keaveney, C. Stewart, Single european sky atm research joint undertaking, SESAR (2019).

[42] R. Clarke, L. B. Moses, The regulation of civilian drones' impacts on public safety, Computer law \& security review 30 (3) (2014) 263-285.

[43] K. V. Andersen, M. H. Frederiksen, M. P. Knudsen, A. D. Krabbe, The strategic responses of start-ups to regulatory constraints in the nascent drone market, Research Policy 49 (10) (2020) 104055.

[44] A. T. Balafoutis, B. Beck, S. Fountas, Z. Tsiropoulos, J. Vangeyte, T. van der Wal, I. Soto-Embodas, M. Gómez-Barbero, S. M. Pedersen, Smart Farming Technologies - Description, Taxonomy and Economic Impact, Springer International Publishing, Cham, 2017, pp. 21-77. doi: $\left\{\$ 10.1007 / 978-3-319-68715-5 \_2 \$\right\}$.

URL https://doi.org/10.1007/978-3-319-68715-5_2

[45] L. Pricewaterhousecoopers, Skies without limits-drones-taking the uk's economy to new heights (2018).

[46] M. Ezuma, F. Erden, C. Kumar Anjinappa, O. Ozdemir, I. Guvenc, Detection and Classification of UAVs Using RF Fingerprints in the Presence of Wi-Fi and Bluetooth Interference, IEEE Open Journal of the Communications Society 1 (2019) 60-76. doi:10.1109/OJCOMS.2019. 2955889

[47] J. Sadovskis, A. Aboltins, J. Eidaks, Modulation recognition of unmanned aerial vehicle control signals using software-defined radio, in: 2018 IEEE 6th Workshop on Advances in Information, Electronic and Electrical Engineering (AIEEE), 2018, pp. 1-5.

[48] P. Kozak, V. Platenka, M. Richterova, Radio Communication Channel Analysis of UAV, in: 2019 International Conference on Military Technologies (ICMT), 2019, pp. 1-4. 
[49] S. Atoev, O.-H. Kwon, S. H. Lee, K. R. Kwon, An efficient SC-FDM modulation technique for a UAV communication link, Electronics 7 (12) (2018) 1-18. doi:10.3390/electronics7120352.

[50] Y. Jia, X. Tu, W. Yan, An UAV Wireless Communication Noise Suppression Method Based on OFDM Modulation and Demodulation, Radio Science 55 (2) (2020) 1-14. doi:10.1029/2019RS006959.

[51] B. Galkin, J. Kibiłda, L. A. DaSilva, Coverage analysis for low-altitude uav networks in urban environments, in: 2017 IEEE Global Communications Conference (GLOBECOM), 2017, pp. 1-6.

[52] V. V. C. Ravi, H. S. Dhillon, Downlink coverage probability in a finite network of unmanned aerial vehicle (uav) base stations, in: Proc. 2016 IEEE 17th International Workshop on Signal Processing Advances in Wireless Communications (SPAWC), Edinburgh, UK, 2016, pp. 1-5.

[53] Y. Li, L. Cai, Uav-assisted dynamic coverage in a heterogeneous cellular system, IEEE Network 31 (4) (2017) 56-61.

[54] M. Mozaffari, W. Saad, M. Bennis, M. Debbah, Drone small cells in the clouds: Design, deployment and performance analysis, in: 2015 IEEE Global Communications Conference (GLOBECOM), 2015, pp. 1-6.

[55] M. Chen, W. Saad, C. Yin, Liquid state machine learning for resource allocation in a network of cache-enabled lte-u uavs, in: 2017 IEEE Global Communications Conference (GLOBECOM), 2017, pp. 1-6.

[56] M. Mozaffari, W. Saad, M. Bennis, M. Debbah, Performance optimization for uav-enabled wireless communications under flight time constraints, in: 2017 IEEE Global Communications Conference (GLOBECOM), 2017, pp. $1-6$.

[57] D. Athukoralage, I. Guvenc, W. Saad, M. Bennis, Regret based learning for uav assisted lte-u/wifi public safety networks, in: 2016 IEEE Global Communications Conference (GLOBECOM), 2016, pp. 1-7.

[58] M. Narang, S. Xiang, W. Liu, J. Gutierrez, L. Chiaraviglio, A. Sathiaseelan, A. Merwaday, Uav-assisted edge infrastructure for challenged networks, in: 2017 IEEE Conference on Computer Communications Workshops (INFOCOM WKSHPS), 2017, pp. 60-65. 
[59] L. Kong, L. Ye, F. Wu, M. Tao, G. Chen, A. V. Vasilakos, Autonomous relay for millimeter-wave wireless communications, IEEE Journal on Selected Areas in Communications 35 (9) (2017) 2127-2136.

[60] W. Qi, W. Hou, L. Guo, Q. Song, A. Jamalipour, A unified routing framework for integrated space/air information networks, IEEE Access 4 (2016) 7084-7103.

[61] G. Gankhuyag, A. P. Shrestha, S.-J. Yoo, Robust and reliable predictive routing strategy for flying ad-hoc networks, IEEE Access 5 (2017) 643654.

[62] S.-Y. Park, D. Jeong, C. S. Shin, H. Lee, Dronenet+: Adaptive route recovery using path stitching of uavs in ad-hoc networks, in: 2017 IEEE Global Communications Conference (GLOBECOM), 2017, pp. 1-7.

[63] O. S. Oubbati, A. Lakas, N. Lagraa, M. B. Yagoubi, Uvar: An intersection uav-assisted vanet routing protocol, in: 2016 IEEE Wireless Communications and Networking Conference (WCNC 2016), Doha, Qatar, 2016, pp. $1-6$.

[64] O. S. Oubbati, A. Lakas, F. Zhou, M. Gunes, N. Lagraa, M. B. Yagoubi, Intelligent uav-assisted routing protocol for urban vanets, Computer Communications 107 (C) (2017) 93-111.

[65] O. S. Oubbati, A. Lakas, F. Zhou, M. Gunes, N. Lagraa, M. B. Yagoubi, Intelligent uav-assisted routing protocol for urban vanets, Computer Communications 107 (C) (2017) 93-111.

[66] M. Gapeyenko, V. Petrov, D. Moltchanov, S. Andreev, N. Himayat, Y. Koucheryavy, Flexible and reliable uav-assisted backhaul operation in $5 \mathrm{~g}$ mmwave cellular networks, IEEE Journal on Selected Areas in Communications 36 (11) (2018) 2486-2496.

[67] R. Motooka, T. Katagiri, S. Murayama, J. Takahashi, Y. Tobe, R. Nishikawa, Distance control between multiple drones for stable communication, in: 2015 IEEE SENSORS, 2015, pp. 1-3.

[68] D. Rautu, R. Dhaou, E. Chaput, Maintaining a permanent connectivity between nodes of an air-to-ground communication network, in: 2017 13th International Wireless Communications and Mobile Computing Conference (IWCMC), 2017, pp. 681-686. 
[69] S. A. W. Shah, T. Khattab, M. Z. Shakir, M. O. Hasna, A distributed approach for networked flying platform association with small cells in $5 \mathrm{~g}+$ networks, in: 2017 IEEE Global Communications Conference (GLOBECOM), 2017, pp. 1-7.

[70] N. Rupasinghe, A. S. Ibrahim, I. Guvenc, Optimum hovering locations with angular domain user separation for cooperative uav networks, in: 2016 IEEE Global Communications Conference (GLOBECOM), 2016, pp. 16.

[71] F. Ahdi, S. Subramaniam, Using unmanned aerial vehicles as relays in wireless balloon networks, in: 2015 IEEE International Conference on Communications (ICC), 2015, pp. 3795-3800.

[72] U. Challita, W. Saad, C. Bettstetter, Interference management for cellularconnected uavs: A deep reinforcement learning approach, IEEE Transactions on Wireless Communications 18 (4) (2019) 2125-2140.

[73] X. Liu, T. Xi, E. Ngai, W. Wang, Path planning for aerial sensor networks with connectivity constraints, in: 2017 IEEE International Conference on Communications (ICC), 2017, pp. 1-6.

[74] M.-A. Messous, S.-M. Senouci, H. Sedjelmaci, Network connectivity and area coverage for uav fleet mobility model with energy constraint, in: Proc. 2016 IEEE Wireless Communications and Networking Conference, Doha, Qatar, 2016, pp. 1-6.

[75] M. Mozaffari, W. Saad, M. Bennis, M. Debbah, Unmanned aerial vehicle with underlaid device-to-device communications: Performance and tradeoffs, IEEE Transactions on Wireless Communications 15 (6) (2016) 3949_ 3963.

[76] M. M. Azari, F. Rosas, K. Chen, S. Pollin, Optimal uav positioning for terrestrial-aerial communication in presence of fading, in: 2016 IEEE Global Communications Conference (GLOBECOM), 2016, pp. 1-7.

[77] U. Challita, W. Saad, Network formation in the sky: Unmanned aerial vehicles for multi-hop wireless backhauling, in: 2017 IEEE Global Communications Conference (GLOBECOM), 2017, pp. 1-6. 
[78] M. Chen, M. Mozaffari, W. Saad, C. Yin, M. Debbah, C. S. Hong, Caching in the sky: Proactive deployment of cache-enabled unmanned aerial vehicles for optimized quality-of-experience, IEEE Journal on Selected Areas in Communications 35 (5) (2017) 1046-1061.

[79] Q. Wu, Y. Zeng, R. Zhang, Joint trajectory and communication design for uav-enabled multiple access, in: 2017 IEEE Global Communications Conference (GLOBECOM), 2017, pp. 1-6.

[80] J. Lyu, Y. Zeng, R. Zhang, Spectrum sharing and cyclical multiple access in uav-aided cellular offloading, in: 2017 IEEE Global Communications Conference (GLOBECOM), 2017, pp. 1-6.

[81] J. Chen, D. Gesbert, Optimal positioning of flying relays for wireless networks: A los map approach, in: 2017 IEEE International Conference on Communications (ICC), 2017, pp. 1-6.

[82] Z. Wang, L. Duan, R. Zhang, Traffic-aware adaptive deployment for uavaided communication networks, in: 2018 IEEE Global Communications Conference (GLOBECOM), Abu Dhabi, United Arab Emirates, United Arab Emirates, 2018, pp. 1-6.

[83] M. Horiuchi, H. Nishiyama, N. Kato, F. Ono, R. Miura, Throughput maximization for long-distance real-time data transmission over multiple uavs, in: 2016 IEEE International Conference on Communications (ICC), 2016, pp. 1-6.

[84] J. Zhang, Y. Zeng, R. Zhang, Spectrum and energy efficiency maximization in uav-enabled mobile relaying, in: 2017 IEEE International Conference on Communications (ICC), 2017, pp. 1-6.

[85] A. Abdulla, Z. M. Fadlullah, H. Nishiyama, N. Kato, F. Ono, R. Miura, An optimal data collection technique for improved utility in uas-aided networks, in: 2014 IEEE International Conference on Computer Communications (INFOCOM), 2014, pp. 736-744.

[86] E. Yanmaz, R. Kuschnig, C. Bettstetter, Channel measurements over 802.11a-based uav-to-ground links, in: 2011 IEEE GLOBECOM Workshops (GC Wkshps), Houston, TX, USA, 2011, pp. 1280-1284. 
[87] H.-F. Yu, X.-F. Chi, J. Liu, An integrated phy-mac analytical model for ieee 802.15.7 vlc network with mpr capability, Optoelectronics Letters 10 (5) (2014) 365-368.

[88] A. Musa, M. D. Baba, H. M. A. H. Mansor, The design and implementation of ieee 802.15.7 module with ns-2 simulator, in: 2014 International Conference on Computer, Communications, and Control Technology (I4CT), 2014, pp. 111-115.

[89] P. Shams, M. Erol-Kantarci, M. Uysal, Mac layer performance of the ieee 802.15.7 visible light communication standard, Transactions on Emerging Telecommunications Technologies 27 (5) (2016) 662-674.

[90] Q. Wang, D. Giustiniano, D. Puccinelli, Openvlc: software-defined visible light embedded networks, in: 1st ACM MobiCom Workshop on Visible Light Communication Systems(VLCS'14), 2014, pp. 15-20.

[91] Y. Takahashiy, Y. Kawamotoy, H. Nishiyamay, N. Katoy, F. F. Onoz, R. Miura, A td-lte-a based efficient radio access scheme for real-time data transmission over relay unmanned aerial vehicle networks, in: 2017 IEEE 86th Vehicular Technology Conference (VTC-Fall), 2017, pp. 1-5.

[92] L. Wang, Y. L. Che, J. Long, L. Duan, K. Wu, Multiple access mmwave design for uav-aided $5 \mathrm{~g}$ communications, IEEE Wireless Communications 26 (1) (2019) 64-71.

[93] S. Say, H. Inata, J. Liu, S. Shimamoto, Priority-based data gathering framework in uav-assisted wireless sensor networks, IEEE Sensors Journal 16 (14) (2016) 5785-5794.

[94] Y. Kawamoto, H. Nishiyama, N. Kato, F. Ono, R. Miura, An efficient throughput-aware resource allocation technique for data transmission in unmanned aircraft systems, in: 2017 IEEE International Conference on Communications (ICC), 2017, pp. 1-6.

[95] H. Feng, L. J. Cimini, On optimum relay deployment in a multi-hop linear network with cooperation, in: MILCOM 2012 - 2012 IEEE Military Communications Conference, 2012, pp. 1-6. 
[96] K. Li, W. Ni, X. Wang, R. P. Liu, S. S. Kanhere, S. Jha, Energy-efficient cooperative relaying for unmanned aerial vehicles, IEEE Transactions on Mobile Computing 15 (6) (2016) 1377-1386.

[97] J. Lee, K. Kim, S. Yoo, A. Y. Chung, J. Y. Lee, S. J. Park, H. Kim, Constructing a reliable and fast recoverable network for drones, in: 2016 IEEE International Conference on Communications (ICC), 2017, pp. 1-6.

[98] W. Qi, W. Hou, Q. Song, L. Guo, A. Jamalipour, Topology control and routing based on adaptive rf/fso switching in space-air integrated networks, in: 2016 IEEE Global Communications Conference (GLOBECOM), 2016, pp. $1-6$.

[99] J. Lu, S. Wan, X. Chen, P. Fan, Energy-efficient 3d uav-bs placement versus mobile users' density and circuit power, in: 2017 IEEE Global Communications Conference (GLOBECOM), 2017, pp. 1-6.

[100] H. Ghazzai, M. B. Ghorbel, A. Kadri, M. J. Hossain, H. Menouar, Energyefficient management of unmanned aerial vehicles for underlay cognitive radio systems, IEEE Transactions on Green Communications and Networking 1 (4) (2017) 434-443.

[101] M. Mozaffari, W. Saad, M. Bennis, M. Debbah, Efficient deployment of multiple unmanned aerial vehicles for optimal wireless coverage, IEEE Communications Letters 20 (8) (2016) 1647-1650.

[102] I. Bor-Yaliniz, A. El-Keyi, H. Yanikomeroglu, Spatial Configuration of Agile Wireless Networks With Drone-BSs and User-in-the-loop, IEEE Transactions on Wireless Communications 18 (2) (2019) 753-768.

[103] J. Li, Y. Han, Optimal resource allocation for packet delay minimization in multi-layer uav networks, IEEE Communications Letters 21 (3) (2017) 580-583.

[104] M. M. Azari, F. Rosas, K.-C. Chen, S. Pollin, Joint sum-rate and power gain analysis of an aerial base station, in: 2016 IEEE Global Communications Conference (GLOBECOM), 2016, pp. 1-6.

[105] N. Goddemeier, C. Wietfeld, Investigation of air-to-air channel characteristics and a uav specific extension to the rice model, in: 2015 IEEE Global Communications Conference (GLOBECOM), 2015, pp. 1-5. 
[106] X. Cai, A. Gonzalez-Plaza, D. Alonso, L. Zhang, C. B. Rodríguez, A. P. Yuste, X. Yin, Low altitude uav propagation channel modelling, in: 2017 11th European Conference on Antennas and Propagation (EUCAP), 2017, pp. 1443-1447.

[107] R. Amorim, H. Nguyen, P. Mogensen, I. Z. Kovacs, J. Wigard, T. B. Sorensen, Radio channel modeling for uav communication over cellular networks, IEEE Wireless Communications Letters 6 (4) (2017) 514-517.

[108] W. Khawaja, O. Ozdemir, I. Guvenc, Uav air-to-ground channel characterization for mmwave systems, in: 2017 IEEE 86th Vehicular Technology Conference (VTC-Fall), 2017, pp. 1-5.

[109] Z. Xiao, P. Xia, X.-G. Xia, Enabling uav cellular with millimeter-wave communication: Potentials and approaches, IEEE Communications Magazine 54 (5) (2016) 66-73.

[110] A. Rahmati, Y. Yapıcı, N. Rupasinghe, I. Guvenc, H. Dai, A. Bhuyany, Energy Efficiency of RSMA and NOMA in Cellular-Connected mmWave UAV Networks, arXiv e-prints (2019) arXiv:1902.04721.

[111] H. Wu, X. Tao, N. Zhang, X. Shen, Cooperative uav cluster-assisted terrestrial cellular networks for ubiquitous coverage, IEEE Journal on Selected Areas in Communications 36 (9) (2018) 2045-2058.

[112] J. Zhao, W. Jia, Efficient channel tracking strategy for mmwave uav communications, Electronics Letters 54 (21) (2018) 1218-1220.

[113] J. Chen, O. Esrafilian, D. Gesbert, U. Mitra, Efficient algorithms for airto-ground channel reconstruction in uav-aided communications, in: 2017 IEEE Global Communications Conference (GLOBECOM), 2017, pp. 1-6.

[114] I. Bor-Yaliniz, S. S. Szyszkowicz, H. Yanikomeroglu, Environment-aware drone-base-station placements in modern metropolitans, IEEE Wireless Communications Letters 7 (3) (2018) 372-375.

[115] V. Vahidi, A. P. Yazdanpanah, E. Saberinia, E. E. Regentova, Channel estimation, equalisation, and evaluation for high-mobility airborne hyperspectral data transmission, IET Communications 10 (8) (2016) 2656-2662. 
[116] D. Darsena, G. Gelli, I. Iudice, F. Verde, Equalization techniques of control and non-payload communication links for unmanned aerial vehicles, IEEE Access 6 (2018) 4485-4496.

[117] C. C. Baseca, J. R. Díaz, J. Lloret, Communication ad hoc protocol for intelligent video sensing using ar drones, in: 2013 IEEE 9th International Conference on Mobile Ad-hoc and Sensor Networks, 2013, pp. 449-453.

[118] A. Orsino, A. Ometov, G. Fodor, D. Moltchanov, L. Militano, S. Andreev, O. N. C. Yilmaz, T. Tirronen, J. Torsner, G. Araniti, A. Iera, M. Dohler, Y. Koucheryavy, Effects of heterogeneous mobility on d2d- and droneassisted mission-critical mtc in 5g, IEEE Communications Magazine 55 (2) (2018) 79-87.

[119] A. Ali, G. Shah, M. Aslam, Model for autonomous agents in machineto-machine navigation networks, International Journal of Communication Systems 31 (4) (2017) 1-13.

[120] W. Fawaz, C. Abou-Rjeily, C. Assi, Uav-aided cooperation for fso communication systems, IEEE Communications Magazine 56 (1) (2018) 70-75.

[121] S. Manfredi, E. Natalizio, C. Pascariello, N. R. Zema, A packet loss tolerant rendezvous algorithm for wireless networked robot systems, Asian Journal of Control 19 (4) (2017) 1413-1423.

[122] S. Mori, Cooperative sensing data collecting framework by using unmanned aircraft vehicle in wireless sensor network, in: 2016 IEEE International Conference on Communications (ICC), 2016, pp. 1-6.

[123] W. Wang, C. Dong, S. Zhu, H. Wang, Dfra: Demodulation-free random access for uav ad hoc networks, in: 2017 IEEE International Conference on Communications (ICC), 2017, pp. 1-6.

[124] Z. Zheng, A. K. Sangaiah, T. Wang, Adaptive communication protocols in flying ad hoc network, IEEE Communications Magazine 56 (1) (2018) 136-142.

[125] P. Kumar, P. Singh, S. Darshi, S. Shailendra, Drone assisted network coded co-operation, in: TENCON 2019 - 2019 IEEE Region 10 Conference (TENCON), 2019, pp. 1174-1179. 
[126] P. Kumar, P. Singh, S. Darshi, S. Shailendra, Analysis of drone assisted network coded cooperation for next generation wireless network, IEEE Transactions on Mobile Computing (2019) 1-11.

[127] M. Zayene, O. Habachi, V. Meghdadi, T. Ezzeddine, J. Cances, Joint delay and energy minimization for instantly decodable network coding, in: 2017 IEEE International Conference on Communications (ICC), 2017, pp. 1-6.

[128] M. Zayene, O. Habachi, V. Meghdadi, T. Ezzeddine, J. Cances, Delay and energy aware instantly decodable network coding for multi-hop cooperative data exchange, in: 2018 IEEE Wireless Communications and Networking Conference (WCNC), 2018, pp. 1-6.

[129] G. Cocco, D. Floreano, Cross-packet coding for delay-constrained streaming applications, IEEE Communications Letters 23 (11) (2019) 1962-1966.

[130] C. Chun, K. M. Jeon, T. Kim, W. Choi, Drone noise reduction using deep convolutional autoencoder for uav acoustic sensor networks, in: 2019 IEEE 16th International Conference on Mobile Ad Hoc and Sensor Systems Workshops (MASSW), 2019, pp. 168-169.

[131] K. Li, W. Ni, X. Wang, R. P. Liu, S. S. Kanhere, S. Jha, Epla: Energybalancing packets scheduling for airborne relaying networks, in: 2015 IEEE International Conference on Communications (ICC), 2015, pp. 62466251.

[132] C. Pu, L. Carpenter, psched: A priority-based service scheduling scheme for the internet of drones, IEEE Systems Journal (2020) 1-10doi:10. $1109 /$ JSYST.2020.2998010.

[133] R. K. X. Ma, R. Dhaou, Fairness-aware uav-assisted data collection in mobile wireless sensor networks, in: 2016 International Wireless Communications and Mobile Computing Conference (IWCMC), 2016, pp. 995-1001.

[134] H. H. Nejad, D. Sauter, S. Aberkane, On-line scheduling and fault detection in ncs with communication constraints in drone application, in: 2010 Conference on Control and Fault-Tolerant Systems (SysTol), 2010, pp. 867872. 
[135] T. Kitagawa, S. Ata, M. Murata, Retrieving information with autonomously-flying routers in information-centric network, in: 2016 IEEE International Conference on Communications (ICC), 2016, pp. 1-6.

[136] X. Ma, S. Chisiu, R. Kacimi, R. Dhaou, Opportunistic communications in wsn using uav, in: 2017 14th IEEE Annual Consumer Communications \& Networking Conference (CCNC), 2017, pp. 510-515.

[137] B. Sliwa, D. Behnke, C. Ide, C. Wietfeld, B.a.t.mobile: Leveraging mobility control knowledge for efficient routing in mobile robotic networks, in: 2016 IEEE Global Communications Conference (GLOBECOM), 2016, pp. $1-6$.

[138] P. Zhang, Q. Zhang, M. Jiang, Z. Feng, Cube based space region partition routing algorithm in uav networks, in: 2017 23rd Asia-Pacific Conference on Communications (APCC), 2017, pp. 1-6.

[139] X. Li, D. Guo, H. Yin, G. Wei, Drone-assisted public safety wireless broadband network, in: 2015 IEEE Wireless Communications and Networking Conference Workshops (WCNCW), 2015, pp. 323-328.

[140] X. Lu, D. Xu, L. Xiao, L. Wang, W. Zhuang, Anti-jamming communication game for uav-aided vanets, in: 2017 IEEE Global Communications Conference (GLOBECOM), 2017, pp. 1-6.

[141] K. Scott, R. Dai, J. Zhang, Online-relaying-based image communication in unmanned aerial vehicle networks, in: 2017 IEEE International Conference on Communications (ICC), 2017, pp. 1-6.

[142] Z. Kaleem, J. Souza, J. Jailton, T. Carvalho, J. Araújo, R. Francês, A proposal for routing protocol for fanet: A fuzzy system approach with qoe/qos guarantee, Wireless Communications and Mobile Computing (2019).

[143] R. Valentino, W.-S. Jung, Y.-B. Ko, A design and simulation of the opportunistic computation offloading with learning-based prediction for unmanned aerial vehicle (uav) clustering networks, Sensors 18 (11) (2018).

[144] R. Valentino, W. Jung, Y. Ko, Opportunistic computational offloading system for clusters of drones, in: 2018 20th International Conference on Advanced Communication Technology (ICACT), 2018, pp. 303-306. 
[145] M. Messous, A. Arfaoui, A. Alioua, S. Senouci, A sequential game approach for computation-offloading in an uav network, in: GLOBECOM 2017 - 2017 IEEE Global Communications Conference, 2017, pp. 1-7.

[146] X. Chen, C. Wu, T. Chen, Z. Liu, M. Bennis, Y. Ji, Age of informationaware resource management in uav-assisted mobile-edge computing systems, in: GLOBECOM 2020 - 2020 IEEE Global Communications Conference, 2020, pp. 1-6. doi:10.1109/GLOBECOM42002.2020. 9322632

[147] W. Jung, J. Yim, Y. Ko, S. Singh, Acods: adaptive computation offloading for drone surveillance system, in: 2017 16th Annual Mediterranean Ad Hoc Networking Workshop (Med-Hoc-Net), 2017, pp. 1-6.

[148] M. A. Messous, H. Sedjelmaci, N. Houari, S. M. Senouci, Computation offloading game for an uav network in mobile edge computing, in: 2017 IEEE International Conference on Communications (ICC), 2017, pp. 1-6.

[149] S. Yaqoob, A. Ullah, M. Awais, I. Katib, A. Albeshri, R. Mehmood, M. Raza, S. ul Islam, J. J. Rodrigues, Novel congestion avoidance scheme for internet of drones, Computer Communications (2021).

[150] X. Hou, Z. Ren, W. Cheng, C. Chen, H. Zhang, Fog based computation offloading for swarm of drones, in: ICC 2019 - 2019 IEEE International Conference on Communications (ICC), 2019, pp. 1-7.

[151] F. Wamser, F. Loh, M. Seufert, P. Tran-Gia, R. Bruschi, P. Lago, Dynamic cloud service placement for live video streaming with a remote-controlled drone, in: 2017 IFIP/IEEE Symposium on Integrated Network and Service Management (IM), 2017, pp. 893-894.

[152] F. Loh, F. Wamser, T. Hoßfeld, P. Tran-Gia, Quality of service assessment of live video streaming with a remote-controlled drone, in: 2018 4th IEEE Conference on Network Softwarization and Workshops (NetSoft), 2018, pp. $462-469$.

[153] S. A. R. Naqvi, S. A. Hassan, H. Pervaiz, Q. Ni, Drone-aided communication as a key enabler for $5 \mathrm{~g}$ and resilient public safety networks, IEEE Communications Magazine 56 (1) (2018) 36-42. 
[154] J. Xu, Y. Zeng, R. Zhang, Uav-enabled wireless power transfer: Trajectory design and energy optimization, IEEE Transactions on Wireless Communications 17 (8) (2018) 5092-5106.

[155] Q. Wu, L. Liu, R. Zhang, Fundamental trade-offs in communication and trajectory design for uav-enabled wireless network, IEEE Wireless Communications 26 (1) (2019) 36-44.

[156] Y. Zeng, R. Zhang, Energy-efficient uav communication with trajectory optimization, IEEE Transactions on Wireless Communications 16 (6) (2017) $3747-3760$.

[157] T. Q. Duong, L. D. Nguyen, L. K. Nguyen, Practical optimisation of path planning and completion time of data collection for uav-enabled disaster communications, in: 2019 15th International Wireless Communications Mobile Computing Conference (IWCMC), 2019, pp. 372-377.

[158] S. Hayat, E. Yanmaz, T. X. Brown, C. Bettstetter, Multi-objective uav path planning for search and rescue, in: 2017 IEEE International Conference on Robotics and Automation (ICRA), 2017, pp. 5569-5574.

[159] Y. Zeng, J. Xu, R. Zhang, Energy minimization for wireless communication with rotary-wing uav, IEEE Transactions on Wireless Communications 18 (4) (2019) 2329-2345.

[160] L. Amorosi, L. Chiaraviglio, F. D'Andreagiovanni, N. Blefari-Melazzi, Energy-efficient mission planning of uavs for $5 \mathrm{~g}$ coverage in rural zones, in: 2018 IEEE International Conference on Environmental Engineering (EE), 2018, pp. 1-9.

[161] L. Li, X. Wen, Z. Lu, Q. Pan, W. Hu, Energy-efficient uav-enabled mec system: Bits allocation optimization and trajectory design, Sensors 19 (2019) $1148-1162$.

[162] L. Chiaraviglio, L. Amorosi, F. Malandrino, C. F. Chiasserini, P. Dell'Olmo, C. Casetti, Optimal throughput management in uav-based networks during disasters, in: IEEE INFOCOM 2019 - IEEE Conference on Computer Communications Workshops (INFOCOM WKSHPS), 2019, pp. 307-312. 
[163] A. S. Prasetia, R. Wai, Y. Wen, Y. Wang, Mission-based energy consumption prediction of multirotor uav, IEEE Access 7 (2019) 33055-33063.

[164] H. Y. Jeong, B. D. Song, S. Lee, Truck-drone hybrid delivery routing: Payload-energy dependency and no-fly zones, International Journal of Production Economics 214 (2019) 220-233.

[165] S. Leary, M. Deittert, J. Bookless, Constrained uav mission planning: A comparison of approaches, in: 2011 IEEE International Conference on Computer Vision Workshops (ICCV Workshops), 2011, pp. 2002-2009.

[166] R. Altawy, A. M. Youssef, Security, privacy, and safety aspects of civilian drones: A survey, ACM Trans. Cyber-Phys. Syst. 1 (2) (2016) 7:1-7:25. doi:10.1145/3001836.

URL http://doi.acm.org/10.1145/3001836

[167] B. Siddappaji, K. B. Akhilesh, Role of Cyber Security in Drone Technology, Springer Singapore, Singapore, 2020, pp. 169-178.

[168] C. Bunse, S. Plotz, Security analysis of drone communication protocols, in: Engineering Secure Software and Systems, Springer International Publishing, 2018, pp. 96-107. doi:10.1109/ ICCCNT.2014.6963085.

[169] J.-S. Pleban, R. Band, R. Creutzburg, Hacking and securing the ar.drone 2.0 quadcopter: investigations for improving the security of a toy, in: Proceedings of SPIE - The International Society for Optical Engineering, Vol. 9030, 2014, pp. 9030 - 9030 - 12. doi: $10.1117 / 12.2044868$.

URL https://doi.org/10.1117/12.2044868

[170] Y. Tan, J. Wang, J. Liu, Y. Zhang, Unmanned systems security: Models, challenges, and future directions, IEEE Network 34 (4) (2020) 291-297.

[171] D. He, S. Chan, M. Guizani, Drone-Assisted Public Safety Networks: The Security Aspect, IEEE Communications Magazine 55 (8) (2017) 218-223.

[172] M. Gupta, M. Abdelsalam, S. Khorsandroo, S. Mittal, Security and privacy in smart farming: Challenges and opportunities, IEEE Access 8 (2020) 34564-34584.

[173] M. J. BOYLE, The costs and consequences of drone warfare, International Affairs 89 (1) (2013) 1-29. doi:10.1111/1468-2346.12002. URL https: / /doi.org/10.1111/1468-2346.12002 
[174] C. Pu, Y. Li, Lightweight authentication protocol for unmanned aerial vehicles using physical unclonable function and chaotic system, in: 2020 IEEE International Symposium on Local and Metropolitan Area Networks (LANMAN), 2020, pp. 1-6.

[175] J. Gordon, V. Kraj, J. H. Hwang, A. Raja, A security assessment for consumer wifi drones, in: 2019 IEEE International Conference on Industrial Internet (ICII), 2019, pp. 1-5.

[176] G. Ding, Q. Wu, L. Zhang, Y. Lin, T. A. Tsiftsis, Y. D. Yao, An amateur drone surveillance system based on the cognitive internet of things, IEEE Communications Magazine 56 (1) (2018) 29-35.

[177] J. Won, S. H. Seo, E. Bertino, A secure communication protocol for drones and smart objects, in: 2015 10th ACM Symposium on Information, Computer and Communications Security (ASIA CCS '15), 2015, pp. 249-260.

[178] P. Gope, B. Sikdar, An efficient privacy-preserving authenticated key agreement scheme for edge-assisted internet of drones, IEEE Transactions on Vehicular Technology 69 (11) (2020) 13621-13630. doi:10.1109/TVT. 2020.3018778 .

[179] M. Tanveer, A. H. Zahid, M. Ahmad, A. Baz, H. Alhakami, Lake-iod: Lightweight authenticated key exchange protocol for the internet of drone environment, IEEE Access 8 (2020) 155645-155659. doi:10.1109/ ACCESS.2020.3019367

[180] A. Hermosilla, A. M. Zarca, J. B. Bernabe, J. Ortiz, A. Skarmeta, Security orchestration and enforcement in nfv/sdn-aware uav deployments, IEEE Access 8 (2020) 131779-131795.

[181] E. Bassi, European drones regulation: Today's legal challenges, in: 2019 International Conference on Unmanned Aircraft Systems (ICUAS), 2019, pp. $443-450$.

[182] C. Lin, D. He, N. Kumar, K. K. R. Choo, A. Vinel, X. Huang, Security and privacy for the internet of drones: Challenges and solutions, IEEE Communications Magazine 56 (1) (2018) 64-69. 
[183] R. Luppicini, A. So, A technoethical review of commercial drone use in the context of governance, ethics, and privacy, Technology in Society 46 (2016) $109-119$. doi:https://doi.org/10.1016/j. techsoc.2016.03.003.

URL http://www.sciencedirect.com/science/article/ pii/S0160791X16300033

[184] M. Sliti, W. Abdallah, N. Boudriga, Jamming attack detection in optical uav networks, in: 2018 20th International Conference on Transparent Optical Networks (ICTON), 2018, pp. 1-5.

[185] X. Lu, L. Xiao, C. Dai, H. Dai, UAV-Aided Cellular Communications with Deep Reinforcement Learning Against Jamming, arXiv e-prints (2018) arXiv:1805.06628arXiv:1805.06628.

[186] Q. Wu, W. Mei, R. Zhang, Safeguarding Wireless Network with UAVs: A Physical Layer Security Perspective, arXiv e-prints (2019) arXiv:1902.02472arXiv:1902.02472.

[187] J. Guth, U. Breitenbücher, M. Falkenthal, F. Leymann, L. Reinfurt, Comparison of iot platform architectures: A field study based on a reference architecture, in: Cloudification of the Internet of Things (CIoT), 2016, pp. 1-6. doi:10.1109/CIOT.2016.7872918.

[188] A. Botta, W. de Donato, V. Persico, A. Pescapé, Integration of cloud computing and internet of things: A survey, Future Generation Computer Systems 56 (2016) $684-700$.

URL http://wWw.sciencedirect.com/science/article/ pii/S0167739X15003015

[189] Y. Zhang, L. Peng, Y. Sun, H. Lu, Editorial: Intelligent industrial iot integration with cognitive computing, Mobile Networks and Applications 23 (2) (2018) 185-187.

URL https://doi.org/10.1007/s11036-017-0939-1

[190] F. Marino, L. Maggiani, L. Nao, P. Pagano, M. Petracca, Towards softwarization in the iot: Integration and evaluation of t-res in the onem $2 \mathrm{~m}$ architecture, in: IEEE Conference on Network Softwarization (NetSoft), 2017, pp. 1-5. 
[191] C. Stergiou, K. E. Psannis, B.-G. Kim, B. Gupta, Secure integration of iot and cloud computing, Future Generation Computer Systems 78 (2018) 964 -975 .

URL http://www.sciencedirect.com/science/article/ pii/S0167739X1630694X

[192] A. Reyna, C. Martín, J. Chen, E. Soler, M. Díaz, On blockchain and its integration with iot. challenges and opportunities, Future Generation Computer Systems 88 (2018) $173-190$.

URL http://www.sciencedirect.com/science/article/ pii/S0167739X17329205

[193] A. M. Cailean, M. Dimian, Impact of ieee 802.15.7 standard on visible light communications usage in automotive applications, IEEE Communications Magazine 55 (4) (2017) 169-175.

[194] S. Rajagopal, R. D. Roberts, S. K. Lim, Ieee 802.15.7 visible light communication: modulation schemes and dimming support, IEEE Communications Magazine 50 (3) (2012) 72-82.

[195] N. Lourenco, D. Terra, N. Kumar, L. N. Alves, R. L. Aguiar, Visible light communication system for outdoor applications, in: 2012 8th International Symposium on Communication Systems, Networks \& Digital Signal Processing (CSNDSP), 2012, pp. 1-6.

[196] S. Wu, H. Wang, C. H. Youn, Visible light communications for $5 \mathrm{~g}$ wireless networking systems: from fixed to mobile communications, IEEE Network 28 (6) (2014) 41-45.

[197] T. Yamazato, I. Takai, H. Okada, T. Fujii, T. Yendo, S. Arai, M. Andoh, T. Harada, K. Yasutomi, K. Kagawa, S. Kawahito, Image-sensor-based visible light communication for automotive applications, IEEE Communications Magazine 52 (7) (2014) 88-97.

[198] P. Boccadoro, M. Losciale, G. Piro, L. A. Grieco, A standardcompliant and information-centric communication platform for the internet of drones, in: Proc. of European Wireless (EW), Catania, Italy, 2018. arXiv:https://telematics.poliba.it/ publications/2018/BoccadoroEW18.pdf. 
[199] A. Shariat, A. Tizghadam, A. Leon-Garcia, An icn-based publish-subscribe platform to deliver uav service in smart cities, in: 2016 IEEE Conference on Computer Communications Workshops (INFOCOM WKSHPS)), 2016, pp. 698-703.

[200] S. Colucci, M. Mongiello, Pushing the role of information in icn, in: 2016 23rd International Conference on Telecommunications (ICT), 2016, pp. 15 .

[201] C. A. Kerrche, F. Ahmad, M. Elhoseny, A. Adnane, Z. Ahmad, B. Nour, Internet of Vehicles Over Named Data Networking: Current Status and Future Challenges, Springer International Publishing, Cham, 2020, pp. 8399.

[202] Z. Sabir, A. Amine, Connected vehicles using ndn for intelligent transportation systems, in: Proceedings of the international conference on industrial engineering and operations management, Vol. 2018, 2018, pp. 2433-2441.

[203] S. Lall, Technological capabilities and industrialization, World Development 20 (2) (1992) 165 - 186. doi:https://doi.org/10.1016/ 0305-750X (92) 90097-F.

URL http://www.sciencedirect.com/science/article/ pii/0305750X9290097F 